\title{
Chemical Reaction of Nitric Oxides with the 5-1DB Defect of the Single-Walled Carbon Nanotube
}

\author{
Lei Vincent Liu, Wei Quan Tian, and Yan Alexander Wang* \\ Department of Chemistry, University of British Columbia, Vancouver, BC V6T 1Z1, Canada \\ Email:yawang@chem.ubc.ca
}

\section{Supplementary Material}

\begin{tabular}{|l|c|}
\hline TABLE OF CONTENTS & Page \\
\hline Figure 1s. Frontier molecular orbitals of $\mathrm{C}_{200} \mathrm{H}_{20}$ & 2 \\
\hline Figure 2s. Frontier molecular orbitals of $\mathrm{C}_{199} \mathrm{H}_{20}$ & 3 \\
\hline Figure 3s. Frontier molecular orbitals of TS1 and LM3 & 4 \\
\hline Figure 4s. Frontier molecular orbitals of the N-substitutionally doped (5,5) SWCNT & 5 \\
\hline Table 1s. Comparison of the energies of LM1, LM2, and LM3 in different schemes & 6 \\
\hline Structure of LM1 within the full DFT scheme & 7 \\
\hline Structure of LM1 within the ONIOM scheme & 11 \\
\hline Structure of LM2 within the full DFT scheme & 15 \\
\hline Structure of LM2 within the ONIOM scheme & 19 \\
\hline Structure of LM3 within the full DFT scheme & 23 \\
\hline Structure of LM3 within the ONIOM scheme & 27 \\
\hline Structure of C $199 \mathrm{H}_{20}$ within the full DFT scheme & 31 \\
\hline Structure of TS1 within the ONIOM scheme & 35 \\
\hline Structure of TS2 within the ONIOM scheme & 39 \\
\hline Structure of TS3 within the ONIOM scheme & 43 \\
\hline Structure of TS4 within the ONIOM scheme & 47 \\
\hline
\end{tabular}




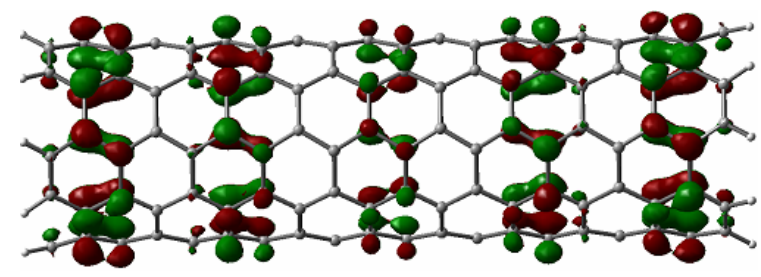

(a) $\mathrm{HOMO}-2$ of $\mathrm{C}_{200} \mathrm{H}_{20}(-4.91 \mathrm{eV})$

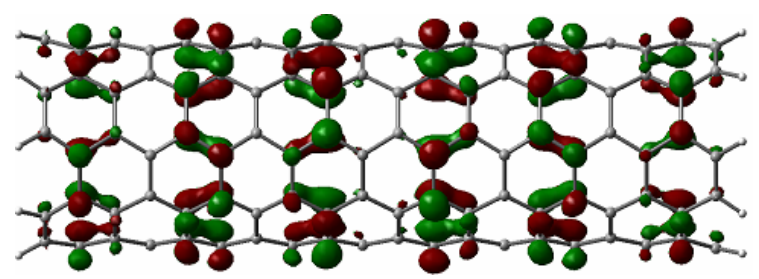

(c) $\mathrm{HOMO}$ of $\mathrm{C}_{200} \mathrm{H}_{20}(-4.35 \mathrm{eV})$

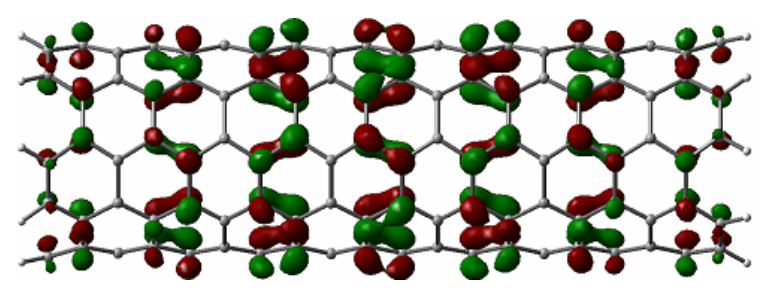

(e) $\mathrm{LUMO}+1$ of $\mathrm{C}_{200} \mathrm{H}_{20}(-2.93 \mathrm{eV})$

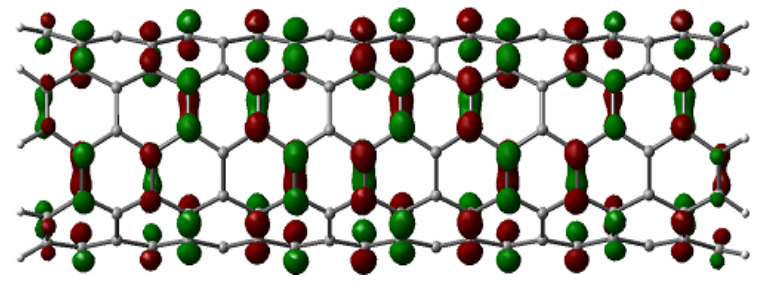

(b) $\mathrm{HOMO}-1$ of $\mathrm{C}_{200} \mathrm{H}_{20}(-4.39 \mathrm{eV})$

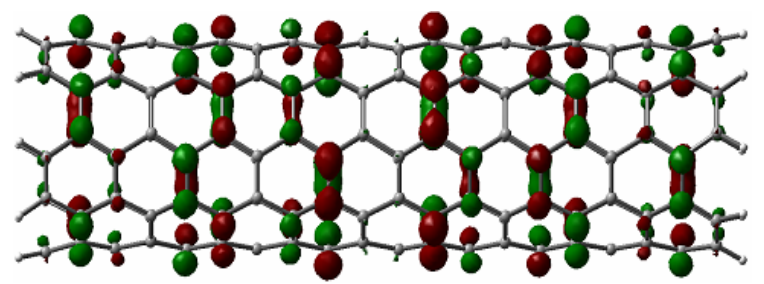

(d) $\mathrm{LUMO}$ of $\mathrm{C}_{200} \mathrm{H}_{20}(-2.97 \mathrm{eV})$

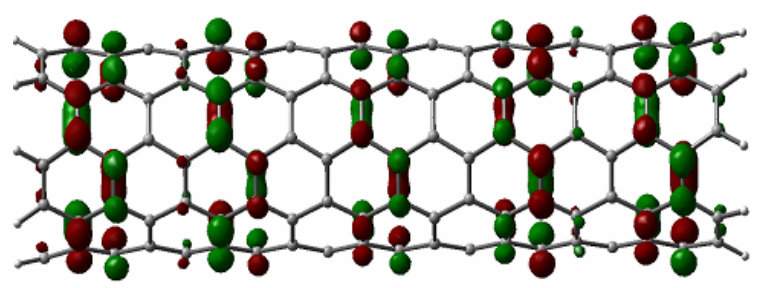

(f) $\mathrm{LUMO}+2$ of $\mathrm{C}_{200} \mathrm{H}_{20}(-2.39 \mathrm{eV})$

Figure 1s. Frontier molecular orbitals of $\mathrm{C}_{200} \mathrm{H}_{20}$. HOMO- $n(p \mathrm{eV})$ is the $n$th molecular orbital below the HOMO with orbital energy $p \mathrm{eV}$. LUMO $+m(q \mathrm{eV})$ is the $m$ th molecular orbital above the LUMO with orbital energy $q \mathrm{eV}$. 


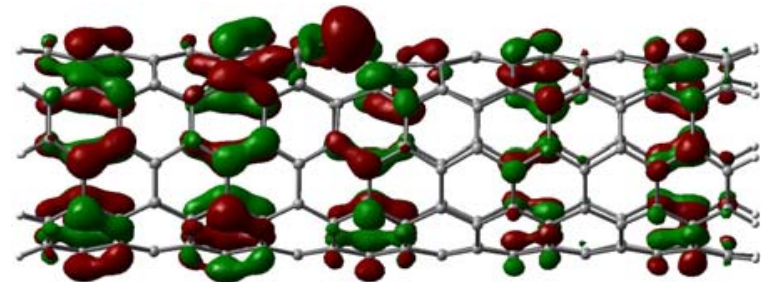

(a) $\mathrm{HOMO}-2$ of $\mathrm{C}_{199} \mathrm{H}_{20}(-5.01 \mathrm{eV})$

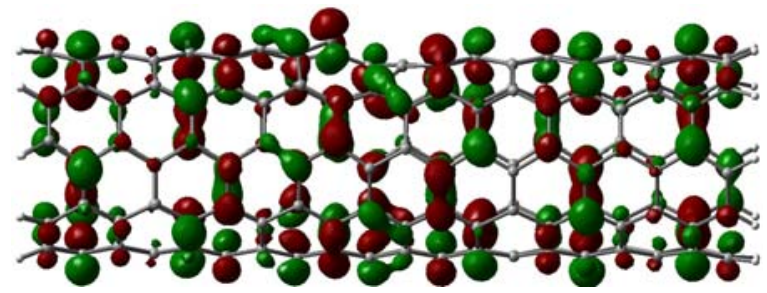

(c) $\mathrm{LUMO}+1$ of $\mathrm{C}_{199} \mathrm{H}_{20}(-3.05 \mathrm{eV})$

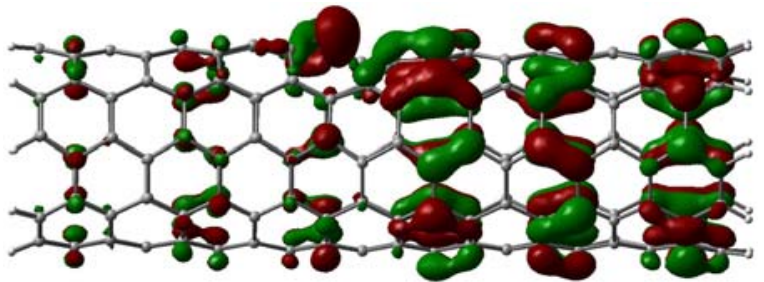

(b) $\mathrm{HOMO}-1$ of $\mathrm{C}_{199} \mathrm{H}_{20}(-4.45 \mathrm{eV})$

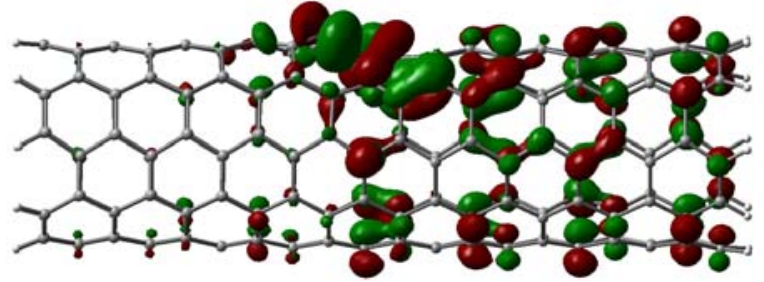

(d) $\mathrm{LUMO}+2$ of $\mathrm{C}_{199} \mathrm{H}_{20}(-2.92 \mathrm{eV})$

Figure 2s. Frontier molecular orbitals of $\mathrm{C}_{199} \mathrm{H}_{20}$. HOMO- $n(p \mathrm{eV})$ is the $n$th molecular orbital below the HOMO with orbital energy $p \mathrm{eV}$. LUMO $+m(q \mathrm{eV})$ is the $m$ th molecular orbital above the LUMO with orbital energy $q \mathrm{eV}$. 


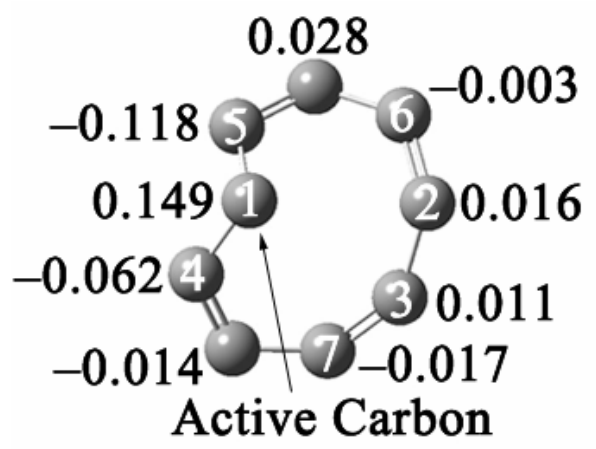

(a) NBO partial charges

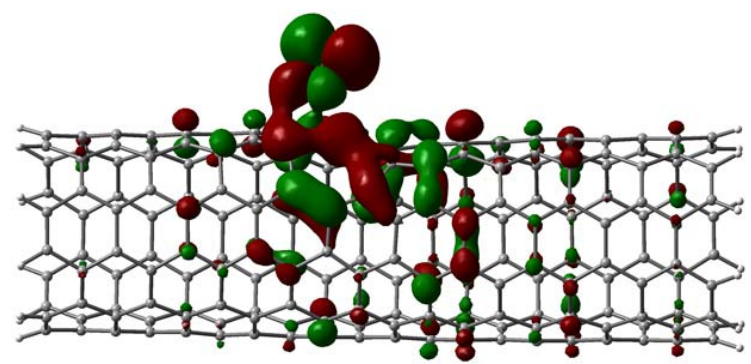

(d) LUMO of TS1 $(-3.08 \mathrm{eV})$

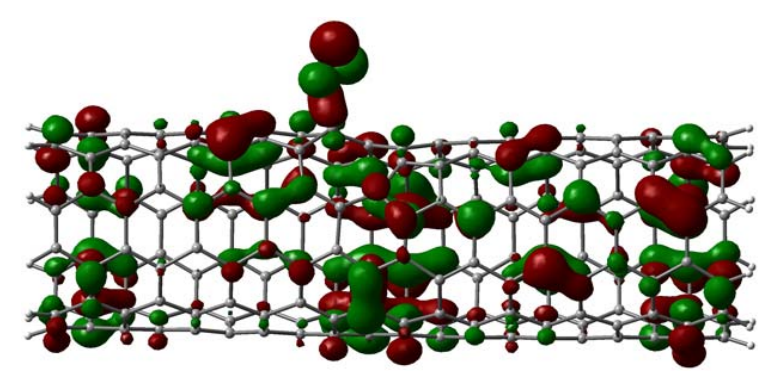

(g) HOMO-18 of TS1 $(-6.49 \mathrm{eV})$

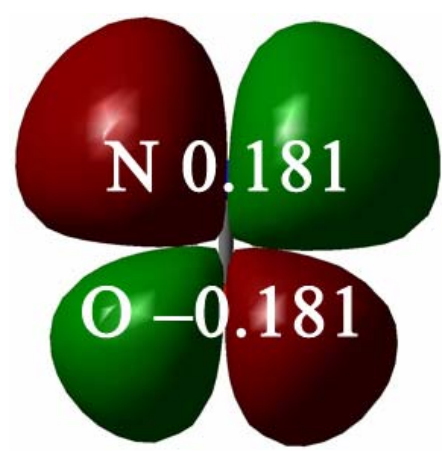

(b) SOMO of NO $(-3.22 \mathrm{eV})$

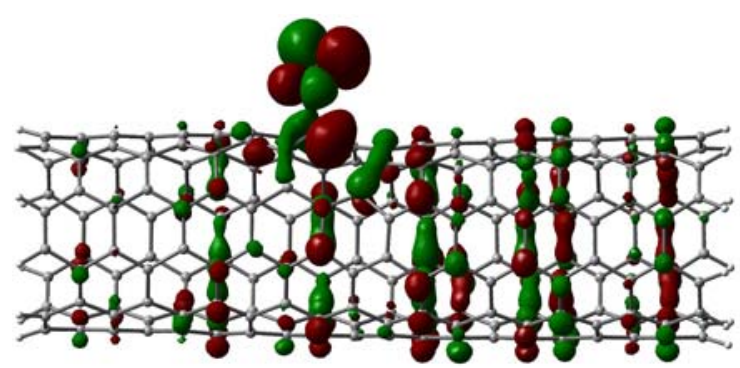

(c) LUMO+2 of TS1 $(-2.80 \mathrm{eV})$

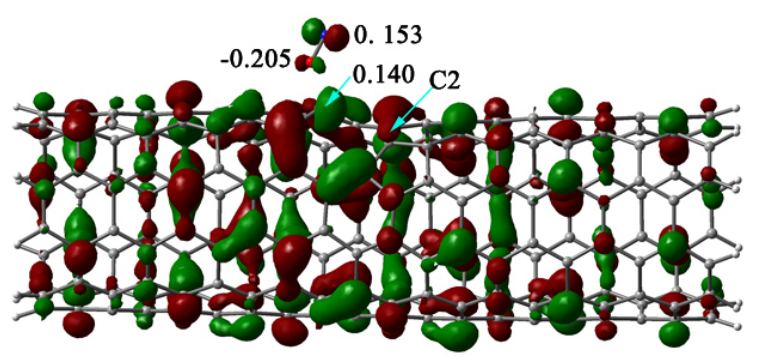

(e) HOMO of TS1 $(-4.66 \mathrm{eV})$ and NBO charges

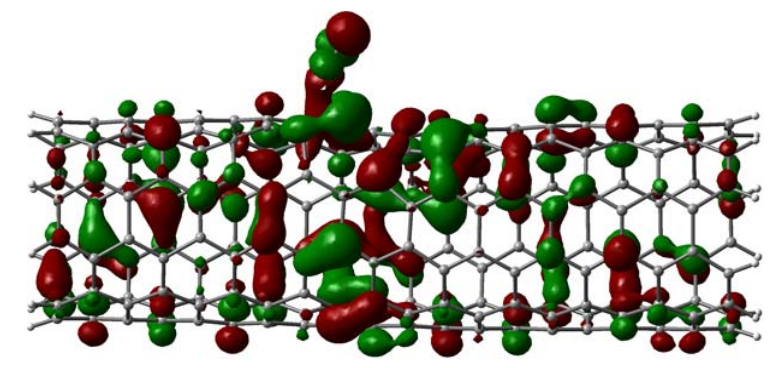

(f) HOMO -7 of TS1 $(-5.86 \mathrm{eV})$

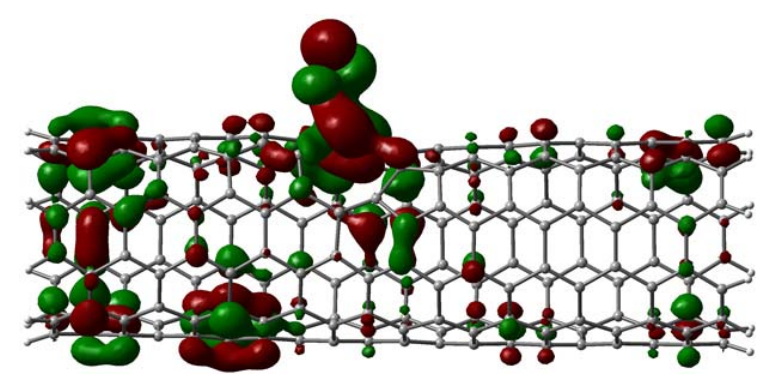

(h) HOMO-19 of TS1 $(-6.54 \mathrm{eV})$

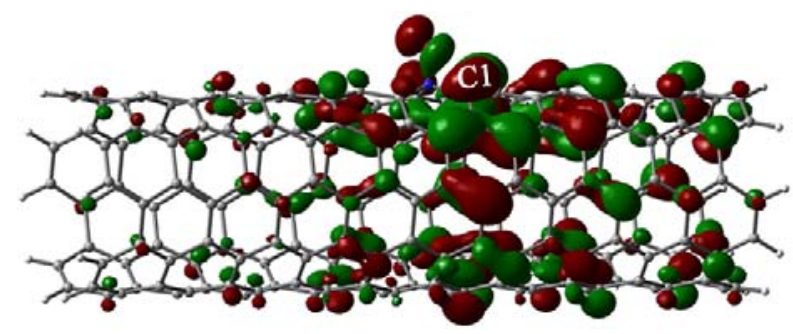

(i) HOMO of LM3 $(-4.56 \mathrm{eV})$

Figure 3s. (a) NBO partial charges of the carbon atoms in the 9-membered ring (adapted from the whole structure calculated at B3LYP/6-31G level of theory); (b) The LUMO of NO and NBO partial charges calculated at the B3LYP/6-31G level of theory. HOMO $-n(p$ eV) is the $n$th occupied molecular orbital below the HOMO with orbital energy $p$ eV. 


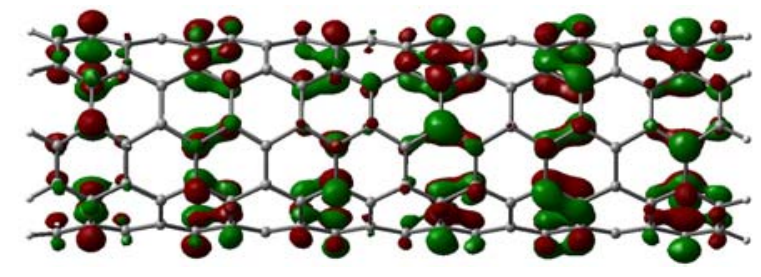

(a) $\mathrm{HOMO}-2(-4.52 \mathrm{eV})$

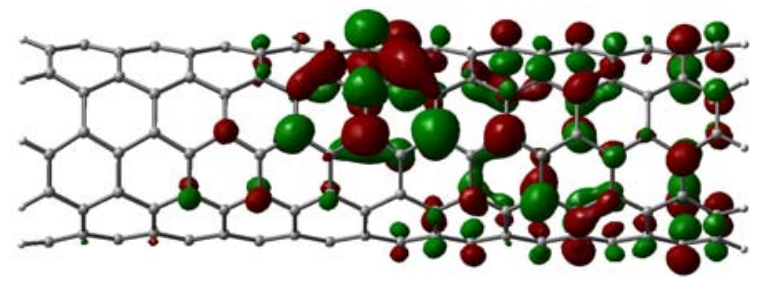

(c) $\mathrm{LUMO}+1(-2.72 \mathrm{eV})$

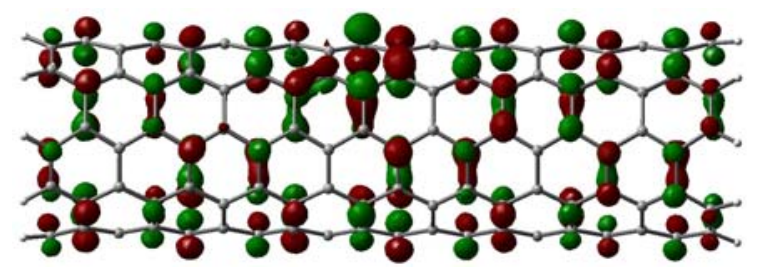

(b) $\mathrm{HOMO}^{-1}(-4.33 \mathrm{eV})$

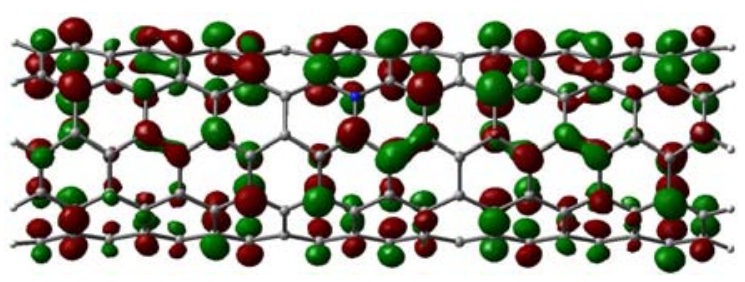

(d) $\mathrm{LUMO}+2(-2.42 \mathrm{eV})$

Figure 4s. Frontier molecular orbitals of the N-substitutionally doped $(5,5)$ SWCNT. HOMO $-n(p$ eV) is the $n$th occupied molecular orbital below the HOMO with orbital energy $p$ eV and LUMO $+m(q \mathrm{eV})$ is the $m$ th occupied molecular orbital above the LUMO with orbital energy $q \mathrm{eV}$. 


\begin{tabular}{|c|c|c|c|c|c|c|}
\hline States & $\begin{array}{c}\text { Full DFT } \\
\mathbf{( H a r t r e e )}\end{array}$ & $\begin{array}{c}\text { Relative Energy } \\
\mathbf{( k c a l / m o l )}\end{array}$ & $\begin{array}{c}\text { ONIOM/DFT } \\
\mathbf{( H a r t r e e )}\end{array}$ & $\begin{array}{c}\text { Relative Energy } \\
\mathbf{( k c a l / m o l )}\end{array}$ & $\begin{array}{c}\text { ONIOM } \\
\mathbf{( H a r t r e e )}\end{array}$ & $\begin{array}{c}\text { Relative Energy } \\
\mathbf{( k c a l / m o l )}\end{array}$ \\
\hline LM1 & -7723.7009 & 0.0 & -7723.4888 & 0.0 & -475.3053 & 0.0 \\
\hline LM2 & -7723.8061 & -66.0 & -7723.6056 & -73.3 & -475.3660 & -38.1 \\
\hline LM3 & -7723.8072 & -66.7 & -7723.6018 & -70.9 & -475.3576 & -32.8 \\
\hline
\end{tabular}

Table 1s. Comparison of the energies of LM1, LM2, and LM3 in the full DFT, ONIOM/DFT, and ONIOM schemes. The relative energies are measured from those of LM1. In the full DFT scheme, the energies and structures are obtained from fully self-consistent DFT optimizations. In the ONIOM scheme, the energies and structures are from fully self-consistent ONIOM optimizations. In the ONIOM/DFT scheme, the structures are from fully self-consistent ONIOM optimizations and the energies are from single-point DFT calculations based on the ONIOM geometries. 


\section{XYZ Coordinates of Structures in $\AA$}

Structure of LM1 within the full DFT scheme

\begin{tabular}{|c|c|c|c|}
\hline & -10.43600 & -1.35109 & 3.33806 \\
\hline$r$ & -10.73186 & -1.81025 & 1.99860 \\
\hline 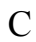 & -11.02017 & -0.98507 & -0.69352 \\
\hline $\mathrm{C}$ & -10.97114 & 0.16581 & -1.56732 \\
\hline $\mathrm{C}$ & -10.56266 & 2.96279 & -1.61898 \\
\hline $\mathrm{C}$ & -10.27352 & 4.11527 & -0.79522 \\
\hline $\mathrm{C}$ & -9.71167 & 5.00825 & 1.83862 \\
\hline $\mathrm{C}$ & -9.52092 & 4.59284 & 3.21057 \\
\hline $\mathrm{C}$ & -9.55909 & 2.33552 & 4.90710 \\
\hline $\mathrm{C}$ & -9.78901 & 0.90863 & 4.92339 \\
\hline $\mathrm{C}$ & -8.74491 & 0.05463 & 4.47264 \\
\hline $\mathrm{C}$ & -9.07596 & -1.10333 & 3.67682 \\
\hline $\mathrm{C}$ & -8.03402 & -1.72696 & 2.91848 \\
\hline $\mathrm{C}$ & -8.32370 & -2.18442 & 1.60645 \\
\hline $\mathrm{C}$ & -9.64690 & -1.99390 & 1.09482 \\
\hline C & -9.79054 & -1.56391 & -0.27593 \\
\hline C & -8.59728 & -1.32613 & -1.03667 \\
\hline C & -8.54306 & -0.18053 & -1.87368 \\
\hline C & -9.69419 & 0.67140 & -1.94299 \\
\hline C & -9.48568 & 2.09923 & -1.97328 \\
\hline C & -8.13967 & 2.59015 & -1.94399 \\
\hline $\mathrm{C}$ & -7.85937 & 3.74450 & -1.16558 \\
\hline C & -8.92292 & 4.34500 & -0.41252 \\
\hline $\mathrm{C}$ & -8.64091 & 4.80192 & 0.92695 \\
\hline $\mathrm{C}$ & -7.31126 & 4.63650 & 1.42888 \\
\hline $\mathrm{C}$ & -7.11859 & 4.20499 & 2.76799 \\
\hline $\mathrm{C}$ & -8.28555 & 3.97432 & 3.56646 \\
\hline C & -8.30785 & 2.81300 & 4.42310 \\
\hline $\mathrm{C}$ & -7.16872 & 1.94101 & 4.40415 \\
\hline $\mathrm{C}$ & -7.39576 & 0.53947 & 4.43535 \\
\hline $\mathrm{C}$ & -6.33713 & -0.34168 & 4.03702 \\
\hline C & -6.66350 & -1.51186 & 3.28348 \\
\hline $\mathrm{C}$ & -5.63645 & -2.17317 & 2.55005 \\
\hline $\mathrm{C}$ & -5.92775 & -2.64056 & 1.21877 \\
\hline $\mathrm{C}$ & -7.23384 & -2.41592 & 0.69821 \\
\hline $\mathrm{C}$ & -7.36879 & -1.96109 & -0.65092 \\
\hline $\mathrm{C}$ & -6.18710 & -1.73062 & -1.41639 \\
\hline $\mathrm{C}$ & -6.12375 & -0.55084 & -2.24030 \\
\hline C & -7.25733 & 0.31475 & -2.28611 \\
\hline 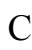 & -7.04994 & 1.72873 & -2.32263 \\
\hline C & -5.71343 & 2.23243 & -2.30831 \\
\hline C & -5.43649 & 3.43331 & -1.56617 \\
\hline$C$ & -6.49897 & 4.02937 & -0.81659 \\
\hline$C$ & -6.23035 & 4.48871 & 0.50843 \\
\hline 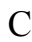 & -4.91068 & 4.36067 & 1.02088 \\
\hline 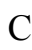 & -4.69235 & 3.87015 & 2.35026 \\
\hline 0 & -5.85782 & 3.60434 & 3.13588 \\
\hline 0 & -5.89362 & 2.43201 & 3.94929 \\
\hline 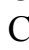 & -4.76357 & 1.56015 & 3.93023 \\
\hline 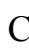 & -4.99768 & 0.14201 & 4.00233 \\
\hline $\mathrm{C}$ & -3.95443 & -0.74651 & 3.61824 \\
\hline & -4.27572 & -1.93972 & 2.90011 \\
\hline & .23664 & -2.60563 & 2.16 \\
\hline
\end{tabular}




\begin{tabular}{|c|c|c|c|}
\hline $\mathrm{C}$ & -3.51865 & -3.06088 & 0.84189 \\
\hline $\mathrm{C}$ & -4.83740 & -2.84111 & 0.31549 \\
\hline $\mathrm{C}$ & -4.96669 & -2.36331 & -1.02488 \\
\hline $\mathrm{C}$ & -3.76821 & -2.11472 & -1.77695 \\
\hline $\mathrm{C}$ & -3.69935 & -0.92496 & -2.56541 \\
\hline $\mathrm{C}$ & -4.83709 & -0.04988 & -2.61175 \\
\hline $\mathrm{C}$ & -4.62288 & 1.36342 & -2.62907 \\
\hline $\mathrm{C}$ & -3.27139 & 1.85438 & -2.55477 \\
\hline $\mathrm{C}$ & -2.99728 & 3.04685 & -1.83876 \\
\hline $\mathrm{C}$ & -4.09126 & 3.72847 & -1.20561 \\
\hline $\mathrm{C}$ & -3.84642 & 4.25082 & 0.10236 \\
\hline $\mathrm{C}$ & -2.51974 & 4.17643 & 0.60684 \\
\hline $\mathrm{C}$ & -2.26217 & 3.48007 & 1.81551 \\
\hline $\mathrm{C}$ & -3.43696 & 3.21034 & 2.63411 \\
\hline $\mathrm{C}$ & -3.50545 & 2.02622 & 3.42726 \\
\hline $\mathrm{C}$ & -2.38175 & 1.14089 & 3.41562 \\
\hline $\mathrm{C}$ & -2.60984 & -0.26246 & 3.56599 \\
\hline $\mathrm{C}$ & -1.56829 & -1.18912 & 3.26451 \\
\hline $\mathrm{C}$ & -1.87969 & -2.38705 & 2.53885 \\
\hline $\mathrm{C}$ & -0.82944 & -3.01856 & 1.80739 \\
\hline $\mathrm{C}$ & -1.10777 & -3.46289 & 0.47437 \\
\hline $\mathrm{C}$ & -2.42182 & -3.26217 & -0.05429 \\
\hline $\mathrm{C}$ & -2.55195 & -2.76909 & -1.39992 \\
\hline $\mathrm{C}$ & -1.36133 & -2.51007 & -2.14143 \\
\hline $\mathrm{C}$ & -1.29111 & -1.30580 & -2.91288 \\
\hline $\mathrm{C}$ & -2.41173 & -0.42502 & -2.93364 \\
\hline $\mathrm{C}$ & -2.18301 & 0.99199 & -2.89334 \\
\hline $\mathrm{C}$ & -0.84086 & 1.4 & -2.78948 \\
\hline $\mathrm{C}$ & -0.54012 & 2.6 & -1. \\
\hline $\mathrm{C}$ & -1.6 & 3.3 & -1 \\
\hline $\mathrm{C}$ & -1.49497 & 4.2 & -0 . \\
\hline $\mathrm{C}$ & 0.2 & 2.8 & 1. \\
\hline $\mathrm{C}$ & -0.99306 & 2.7 & 262 \\
\hline $\mathrm{C}$ & -1.10668 & 1.58886 & 313 \\
\hline $\mathrm{C}$ & -0.01815 & 0.6 & 149 \\
\hline $\mathrm{C}$ & -0.2 & -0.7 & 961 \\
\hline $\mathrm{C}$ & 0.8 & -1 . & 93 \\
\hline $\mathrm{C}$ & 0.5 & -2. & 34 \\
\hline $\mathrm{C}$ & 1.5 & -3 & 78 \\
\hline $\mathrm{C}$ & 1.3 & -3 . & 568 \\
\hline $\mathrm{C}$ & -0.00035 & -3.63526 & -0.42377 \\
\hline $\mathrm{C}$ & -0.13247 & -3.14706 & -1.76053 \\
\hline $\mathrm{C}$ & 1.05617 & -2.88257 & -2.51122 \\
\hline $\mathrm{C}$ & 1.12129 & -1.67084 & -3.28202 \\
\hline $\mathrm{C}$ & -0.00372 & -0.79856 & -3.26852 \\
\hline $\mathrm{C}$ & 0.22531 & 0.60627 & -3.16831 \\
\hline $\mathrm{C}$ & 1.55980 & 1.09697 & -3.09494 \\
\hline $\mathrm{C}$ & 1.85051 & 2.20505 & -2.22147 \\
\hline $\mathrm{C}$ & 0.77392 & 2.76627 & -1.45015 \\
\hline $\mathrm{C}$ & 1.05760 & 3.44291 & -0.07179 \\
\hline $\mathrm{C}$ & 2.51849 & 3.19101 & 0.25833 \\
\hline $\mathrm{C}$ & 2.66020 & 2.60014 & 1.51280 \\
\hline $\mathrm{C}$ & 1.38383 & 2.26214 & 2.03824 \\
\hline $\mathrm{C}$ & 1.28784 & 1.13303 & 2.87736 \\
\hline $\mathrm{C}$ & 2.42855 & 0.26939 & 3.02540 \\
\hline $\mathrm{C}$ & 2.19058 & -1.14818 & 3.02127 \\
\hline $\mathrm{C}$ & 3.25577 & -2.02394 & 2.64648 \\
\hline
\end{tabular}




\begin{tabular}{|c|c|c|c|}
\hline $\mathrm{C}$ & 2.94706 & -3.16670 & 1.83216 \\
\hline $\mathrm{C}$ & 4.00562 & -3.77014 & 1.07290 \\
\hline $\mathrm{C}$ & 3.73290 & -4.18721 & -0.26546 \\
\hline $\mathrm{C}$ & 2.41262 & -3.99744 & -0.79163 \\
\hline $\mathrm{C}$ & 2.27893 & -3.50572 & -2.13312 \\
\hline $\mathrm{C}$ & 3.46911 & -3.22106 & -2.88056 \\
\hline $\mathrm{C}$ & 3.52671 & -2.02158 & -3.65492 \\
\hline $\mathrm{C}$ & 2.39415 & -1.14877 & -3.64761 \\
\hline $\mathrm{C}$ & 2.61955 & 0.26767 & -3.56754 \\
\hline $\mathrm{C}$ & 3.96044 & 0.75841 & -3.55813 \\
\hline $\mathrm{C}$ & 4.26618 & 1.89926 & -2.74672 \\
\hline $\mathrm{C}$ & 3.23267 & 2.50333 & -1.96854 \\
\hline $\mathrm{C}$ & 3.56249 & 3.01107 & -0.66136 \\
\hline $\mathrm{C}$ & 4.87912 & 2.73549 & -0.14417 \\
\hline $\mathrm{C}$ & 5.02508 & 2.21966 & 1.18232 \\
\hline $\mathrm{C}$ & 3.84370 & 1.96107 & 1.93395 \\
\hline $\mathrm{C}$ & 3.73044 & 0.75005 & 2.69701 \\
\hline $\mathrm{C}$ & 4.85066 & -0.14199 & 2.71607 \\
\hline $\mathrm{C}$ & 4.61039 & -1.55065 & 2.68792 \\
\hline $\mathrm{C}$ & 5.67759 & -2.42405 & 2.29440 \\
\hline $\mathrm{C}$ & 5.36672 & -3.55738 & 1.46393 \\
\hline $\mathrm{C}$ & 6.41348 & -4.15227 & 0.70212 \\
\hline $\mathrm{C}$ & 6.13953 & -4.55366 & -0.64943 \\
\hline $\mathrm{C}$ & 4.83148 & -4.35504 & -1.16963 \\
\hline $\mathrm{C}$ & 4.69582 & -3.85529 & -2.51281 \\
\hline $\mathrm{C}$ & 5.87367 & -3.57694 & -3.26067 \\
\hline $\mathrm{C}$ & 5.92638 & -2.38084 & -4.05408 \\
\hline $\mathrm{C}$ & 4.80128 & -1.51038 & -4.05188 \\
\hline $\mathrm{C}$ & 5.02167 & -0.08883 & -4.01378 \\
\hline $\mathrm{C}$ & 6.36085 & 0.39424 & -4.00839 \\
\hline $\mathrm{C}$ & 6.67999 & 1.52724 & -3.18030 \\
\hline $\mathrm{C}$ & 5.64890 & 2.13071 & -2.4 \\
\hline $\mathrm{C}$ & 5.95841 & 2.53851 & -1.06237 \\
\hline $\mathrm{C}$ & 7.26574 & 2.29505 & -0.55256 \\
\hline $\mathrm{C}$ & 7.40783 & 1.78779 & 0.7 \\
\hline $\mathrm{C}$ & 6.23 & 1.5 & 1.5 \\
\hline $\mathrm{C}$ & 6.14 & 0.3 & 2.3 \\
\hline $\mathrm{C}$ & 7.26 & -0.5 & 162 \\
\hline $\mathrm{C}$ & 7.0 & -1.95340 & 2.33285 \\
\hline $\mathrm{C}$ & 8.08509 & -2.81700 & 1.90952 \\
\hline $\mathrm{C}$ & 7.77682 & -3.92872 & 1.08046 \\
\hline $\mathrm{C}$ & 8.83710 & -4.53251 & 0.32119 \\
\hline $\mathrm{C}$ & 8.56252 & -4.91740 & -1.04173 \\
\hline $\mathrm{C}$ & 7.24279 & -4.69019 & -1.55501 \\
\hline $\mathrm{C}$ & 7.10813 & -4.19296 & -2.87873 \\
\hline $\mathrm{C}$ & 8.29628 & -3.93970 & -3.64224 \\
\hline $\mathrm{C}$ & 8.34450 & -2.74969 & -4.45607 \\
\hline $\mathrm{C}$ & 7.20597 & -1.87466 & -4.44586 \\
\hline $\mathrm{C}$ & 7.42386 & -0.46947 & -4.42852 \\
\hline $\mathrm{C}$ & 8.77818 & 0.00982 & -4.43203 \\
\hline $\mathrm{C}$ & 9.10154 & 1.13420 & -3.58935 \\
\hline $\mathrm{C}$ & 8.05129 & 1.71686 & -2.80179 \\
\hline $\mathrm{C}$ & 8.34723 & 2.09791 & -1.46546 \\
\hline $\mathrm{C}$ & 9.67338 & 1.86952 & -0.96063 \\
\hline $\mathrm{C}$ & 9.81385 & 1.36234 & 0.38193 \\
\hline $\mathrm{C}$ & 8.62002 & 1.11575 & 1.13879 \\
\hline $\mathrm{C}$ & 8.54723 & -0.05629 & 1.93872 \\
\hline
\end{tabular}




\begin{tabular}{|c|c|c|c|}
\hline $\mathrm{C}$ & 9.68290 & -0.93413 & 1.96756 \\
\hline $\mathrm{C}$ & 9.44480 & -2.35617 & 1.95828 \\
\hline $\mathrm{C}$ & 10.50205 & -3.23356 & 1.59080 \\
\hline $\mathrm{C}$ & 10.18585 & -4.36417 & 0.73474 \\
\hline $\mathrm{C}$ & 9.64630 & -5.10797 & -1.94285 \\
\hline $\mathrm{C}$ & 9.50840 & -4.59761 & -3.29658 \\
\hline $\mathrm{C}$ & 9.60214 & -2.26634 & -4.90777 \\
\hline $\mathrm{C}$ & 9.82515 & -0.82976 & -4.90160 \\
\hline $\mathrm{C}$ & 10.46036 & 1.37001 & -3.24060 \\
\hline $\mathrm{C}$ & 10.75908 & 1.74411 & -1.86721 \\
\hline $\mathrm{C}$ & 11.03478 & 0.74978 & 0.77330 \\
\hline $\mathrm{C}$ & 10.96670 & -0.44052 & 1.60587 \\
\hline $\mathrm{C}$ & -11.01678 & 5.20150 & 1.26459 \\
\hline $\mathrm{H}$ & -11.82988 & & 1.88213 \\
\hline $\mathrm{C}$ & -10.63080 & & 4.09870 \\
\hline $\mathrm{H}$ & -11.51635 & & 4.02070 \\
\hline $\mathrm{C}$ & -10.6 & & \\
\hline $\mathrm{H}$ & -11.5 & & 5.49136 \\
\hline $\mathrm{C}$ & -11.1 & & 260 \\
\hline $\mathrm{H}$ & -11. & & 902 \\
\hline $\mathrm{C}$ & -11.4 & & 4.17952 \\
\hline $\mathrm{H}$ & -12 & & 13 \\
\hline $\mathrm{C}$ & -11.2 & & 53 \\
\hline $\mathrm{H}$ & -12 . & & 68 \\
\hline $\mathrm{C}$ & -11 . & & 76 \\
\hline $\mathrm{H}$ & -12 & & 967 \\
\hline $\mathrm{C}$ & -12 & & -1.70727 \\
\hline $\mathrm{H}$ & -13 & & -1.65930 \\
\hline $\mathrm{C}$ & -12 & & 0.10649 \\
\hline $\mathrm{H}$ & -13 & & 7565 \\
\hline $\mathrm{C}$ & -12 & & 1.41367 \\
\hline $\mathrm{H}$ & -12 & & 2.03937 \\
\hline 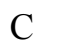 & 12.19 & & -0.00995 \\
\hline $\mathrm{H}$ & 13.1 & & 0.34861 \\
\hline $\mathrm{C}$ & 12.0 & & -1.29241 \\
\hline W & 12.9 & 92 & -1.91285 \\
\hline $\mathrm{C}$ & 11.4 & 0.8 & -4.10336 \\
\hline $\mathrm{H}$ & 12.4 & 1.1 & -4.01761 \\
\hline $\mathrm{C}$ & 11.14469 & -0.26 & -4.91259 \\
\hline $\mathrm{H}$ & 11.94207 & -0.77192 & -5.44795 \\
\hline $\mathrm{C}$ & 12.06149 & -1.36056 & 1.70735 \\
\hline $\mathrm{H}$ & 13.08097 & -0.99568 & 1.64728 \\
\hline $\mathrm{C}$ & 11.83566 & -2.71813 & 1.70228 \\
\hline $\mathrm{H}$ & 12.68238 & -3.39254 & 1.63769 \\
\hline $\mathrm{C}$ & 11.18886 & -5.02059 & -0.05457 \\
\hline $\mathrm{H}$ & 12.20236 & -5.10554 & 0.32157 \\
\hline $\mathrm{C}$ & 10.92742 & -5.37936 & -1.35709 \\
\hline $\mathrm{H}$ & 11.74065 & -5.73924 & -1.97777 \\
\hline $\mathrm{C}$ & 10.64351 & -4.33420 & -4.13255 \\
\hline $\mathrm{H}$ & 11.53240 & -4.94941 & -4.04613 \\
\hline $\mathrm{C}$ & 10.68760 & -3.20357 & -4.91695 \\
\hline $\mathrm{H}$ & 11.61019 & -2.95634 & -5.43029 \\
\hline $\mathrm{O}$ & -0.51034 & 5.17736 & -0.08078 \\
\hline $\mathrm{N}$ & 0.90367 & 4.91637 & -0.17049 \\
\hline
\end{tabular}


Structure of LM1 within the ONIOM scheme

\begin{tabular}{|c|c|c|c|}
\hline$c$ & 803 & 45 & \\
\hline $\mathrm{C}$ & -10.52866 & -3.49384 & 0.37192 \\
\hline S & -10.55439 & -2.62526 & 0945 \\
\hline S & -10.55741 & -1.46745 & -3.12416 \\
\hline S & -10.58979 & 1.34936 & -3.05120 \\
\hline$C$ & -10.61065 & 2.47722 & -2.19429 \\
\hline C & -10.57773 & 3.36636 & 0.49109 \\
\hline $\mathrm{C}$ & -10.51835 & 2.95053 & 84409 \\
\hline $\mathrm{C}$ & -10.42941 & 0.64513 & 3.45081 \\
\hline $\mathrm{C}$ & -10.43407 & -0.76974 & 3.39812 \\
\hline $\mathrm{C}$ & -9.22132 & -1.44772 & 3.14830 \\
\hline $\mathrm{C}$ & -9.25911 & -2.66481 & 2.27827 \\
\hline $\mathrm{C}$ & -8.06401 & 51 & 66682 \\
\hline $\mathrm{C}$ & -8.09317 & 33 & 738 \\
\hline $\mathrm{C}$ & -9.31803 & -3.55372 & -0.35512 \\
\hline $\mathrm{C}$ & -9.32831 & $-3.0^{\prime}$ & 1.77368 \\
\hline $\mathrm{C}$ & -8.11565 & -2.6 & \\
\hline $\mathrm{C}$ & -8.11743 & -1.4 & \\
\hline $\mathrm{C}$ & -9.3 & -0.7 & \\
\hline $\mathrm{C}$ & -9.3 & & \\
\hline $\mathrm{C}$ & -8.1 & & \\
\hline $\mathrm{C}$ & -8.1 & 82 & -2.2 \\
\hline $\mathrm{C}$ & -9.3 & 06 & \\
\hline $\mathrm{C}$ & -9.3 & 0 & \\
\hline $\mathrm{C}$ & -8.1 & 1 & \\
\hline $\mathrm{C}$ & -8.08126 & 3.0 & \\
\hline $\mathrm{C}$ & -9 & 75 & 2.38818 \\
\hline $\mathrm{C}$ & -9.21535 & 7 & 3.22137 \\
\hline $\mathrm{C}$ & -7.98 & 4 & \\
\hline $\mathrm{C}$ & -7.9 & -0.76 & 153 \\
\hline $\mathrm{C}$ & -6.73020 & $-1.4^{7}$ & 3.01080 \\
\hline $\mathrm{C}$ & -6.76618 & -2.65354 & 2.23467 \\
\hline $\mathrm{C}$ & -5.57143 & -3.15322 & 1.67926 \\
\hline $\mathrm{C}$ & -5.59879 & -3.64310 & 0.26 \\
\hline $\mathrm{C}$ & -6.82174 & -3.5 & 02 \\
\hline $\mathrm{C}$ & -6.82987 & -3.0 & 16 \\
\hline $\mathrm{C}$ & -5.61630 & $-2.6^{\prime}$ & -2.34262 \\
\hline $\mathrm{C}$ & -5.61409 & -1.40 & -3.1 \\
\hline $\mathrm{C}$ & -6.835 & -0.7 & \\
\hline $\mathrm{C}$ & -6.848 & & \\
\hline $\mathrm{C}$ & -5.63825 & 1.38 & \\
\hline $\mathrm{C}$ & -5.6 & & \\
\hline $\mathrm{C}$ & -6.9 & & \\
\hline $\mathrm{C}$ & -6.90046 & 3.53 & -0.3 \\
\hline $\mathrm{C}$ & -5.681 & 3.65 & \\
\hline $\mathrm{C}$ & -5.57 & & \\
\hline $\mathrm{C}$ & & & \\
\hline $\mathrm{C}$ & & & \\
\hline $\mathrm{C}$ & & & \\
\hline $\mathrm{C}$ & & & \\
\hline 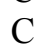 & & & \\
\hline 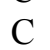 & & & \\
\hline 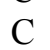 & & & \\
\hline 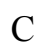 & & & \\
\hline 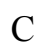 & -4.39155 & -3.61842 & -0.46297 \\
\hline
\end{tabular}




\begin{tabular}{|c|c|c|c|}
\hline $\mathrm{C}$ & -4.39917 & -3.11700 & -1.77951 \\
\hline$c$ & -3.11651 & -2.64186 & -2.35577 \\
\hline $\mathrm{C}$ & -3.11345 & -1.42010 & -3.05599 \\
\hline $\mathrm{C}$ & -4.39860 & -0.70069 & -3.24539 \\
\hline $\mathrm{C}$ & -4.40540 & 0.70560 & -3.17902 \\
\hline $\mathrm{C}$ & -3.12488 & 1.41324 & -2.88471 \\
\hline $\mathrm{C}$ & -3.17214 & 2.61514 & -2.16569 \\
\hline $\mathrm{C}$ & -4.48323 & 3.12527 & -1.68738 \\
\hline $\mathrm{C}$ & -4.51165 & 3.72065 & -0.41105 \\
\hline $\mathrm{C}$ & -3.23141 & 3.79479 & 0.30746 \\
\hline $\mathrm{C}$ & -3.03879 & 3.03396 & 1.41988 \\
\hline $\mathrm{C}$ & -4.30051 & 2.54171 & 2.09923 \\
\hline $\mathrm{C}$ & -4.26925 & 1.33797 & 2.82251 \\
\hline $\mathrm{C}$ & -2.99018 & 0.60340 & 2.94601 \\
\hline $\mathrm{C}$ & -3.02473 & -0.80146 & 3.07904 \\
\hline $\mathrm{C}$ & -1.83793 & -1.54336 & 2.97814 \\
\hline $\mathrm{C}$ & -1.85324 & -2.80197 & 2.17258 \\
\hline $\mathrm{C}$ & -0.64454 & -3.22093 & 1.57581 \\
\hline $\mathrm{C}$ & -0.66703 & -3.68463 & 0.24522 \\
\hline $\mathrm{C}$ & -1.89141 & -3.69643 & -0.45909 \\
\hline $\mathrm{C}$ & -1.89991 & -3.14964 & -1.85242 \\
\hline $\mathrm{C}$ & -0.68469 & -2.65378 & -2.37167 \\
\hline $\mathrm{C}$ & -0.68258 & -1.41696 & -3.04753 \\
\hline $\mathrm{C}$ & -1.89690 & -0.73007 & -3.22838 \\
\hline $\mathrm{C}$ & -1.89162 & 0.75657 & -3.09135 \\
\hline $\mathrm{C}$ & -0.66 & 1.4 & -2.80464 \\
\hline $\mathrm{C}$ & -0.6 & 2.6 & 4466 \\
\hline $\mathrm{C}$ & -1.9 & & 426 \\
\hline $\mathrm{C}$ & -2.0 & & 141 \\
\hline $\mathrm{C}$ & -0.3 & & \\
\hline $\mathrm{C}$ & -1.6 & 2.5 & 322 \\
\hline $\mathrm{C}$ & -1.7 & & 86 \\
\hline $\mathrm{C}$ & -0.5 & & 97 \\
\hline $\mathrm{C}$ & -0. & -0. & 28 \\
\hline $\mathrm{C}$ & & & 07 \\
\hline $\mathrm{C}$ & & 3 & 35 \\
\hline $\mathrm{C}$ & & 52 & 41 \\
\hline $\mathrm{C}$ & & & 44 \\
\hline $\mathrm{C}$ & & 24 & 1571 \\
\hline $\mathrm{C}$ & 0 & & 1281 \\
\hline $\mathrm{C}$ & 1.86730 & & -2.38466 \\
\hline $\mathrm{C}$ & 1.82789 & & -3.07683 \\
\hline $\mathrm{C}$ & 0.59016 & -0.66 & -3.17899 \\
\hline $\mathrm{C}$ & 0.55969 & 0.74032 & -3.01243 \\
\hline $\mathrm{C}$ & 1.76731 & 1.42279 & -2.78140 \\
\hline $\mathrm{C}$ & 1.76256 & 2.59843 & -1.87304 \\
\hline $\mathrm{C}$ & 0.46783 & 2.95439 & -1.23075 \\
\hline $\mathrm{C}$ & 0.46631 & 3.63983 & 0.15441 \\
\hline $\mathrm{C}$ & 1.87571 & 3.63887 & 0.72556 \\
\hline $\mathrm{C}$ & 1.91409 & 2.88945 & 1.95282 \\
\hline $\mathrm{C}$ & 0.74300 & 2.33276 & 2.20209 \\
\hline $\mathrm{C}$ & 0.65058 & 1.16880 & 2.96208 \\
\hline $\mathrm{C}$ & 1.89934 & 0.47778 & 3.38436 \\
\hline $\mathrm{C}$ & 1.90586 & -1.00047 & 3.37070 \\
\hline $\mathrm{C}$ & 3.11260 & -1.67456 & 3.14415 \\
\hline $\mathrm{C}$ & 3.09760 & -2.84012 & 2.22417 \\
\hline 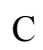 & 4.37663 & -3.23827 & 1.58637 \\
\hline
\end{tabular}




\begin{tabular}{|c|c|c|c|}
\hline $\mathrm{C}$ & 4.36747 & -3.64229 & 0.24457 \\
\hline $\mathrm{C}$ & 3.08834 & -3.62271 & -0.50102 \\
\hline $\mathrm{C}$ & 3.08559 & -3.07015 & -1.80395 \\
\hline $\mathrm{C}$ & 4.36292 & -2.53141 & -2.35575 \\
\hline $\mathrm{C}$ & 4.33023 & -1.30558 & -3.05629 \\
\hline $\mathrm{C}$ & 3.02603 & -0.60586 & -3.19224 \\
\hline $\mathrm{C}$ & 2.98598 & 0.79383 & -3.06716 \\
\hline $\mathrm{C}$ & 4.24339 & 1.53936 & -2.87489 \\
\hline $\mathrm{C}$ & 4.25056 & 2.66795 & -2.04365 \\
\hline $\mathrm{C}$ & 2.98333 & 3.08631 & -1.37012 \\
\hline $\mathrm{C}$ & 3.04321 & 3.60788 & -0.06177 \\
\hline $\mathrm{C}$ & 4.35320 & 3.53146 & 0.65187 \\
\hline $\mathrm{C}$ & 4.39813 & 2.89933 & 1.91314 \\
\hline $\mathrm{C}$ & 3.12225 & 2.42701 & 2.50117 \\
\hline $\mathrm{C}$ & 3.11277 & 1.16701 & 3.27143 \\
\hline $\mathrm{C}$ & 4.38803 & 0.44498 & 3.42998 \\
\hline $\mathrm{C}$ & 4.39236 & -0.95573 & 3.35540 \\
\hline $\mathrm{C}$ & 5.67054 & -1.66198 & 3.11930 \\
\hline $\mathrm{C}$ & 5.66348 & -2.84292 & 2.21251 \\
\hline $\mathrm{C}$ & 6.87014 & -3.23901 & 1.60730 \\
\hline $\mathrm{C}$ & 6.85539 & -3.65449 & 0.17790 \\
\hline $\mathrm{C}$ & 5.63352 & -3.63866 & -0.52122 \\
\hline $\mathrm{C}$ & 5.59566 & -3.02475 & -1.87801 \\
\hline $\mathrm{C}$ & 6.79437 & -2.54182 & -2.43078 \\
\hline $\mathrm{C}$ & 6.75799 & -1.26992 & -3.18749 \\
\hline $\mathrm{C}$ & 5.52920 & -0.60261 & -3.30606 \\
\hline $\mathrm{C}$ & 5.51508 & 0.88675 & -3.25444 \\
\hline $\mathrm{C}$ & 6.72494 & 1.57897 & -3.06895 \\
\hline $\mathrm{C}$ & 6.74458 & 2.77161 & -2.17833 \\
\hline $\mathrm{C}$ & 5.55099 & 3.17757 & -1.54751 \\
\hline $\mathrm{C}$ & 5.60290 & 3.62301 & -0.13267 \\
\hline $\mathrm{C}$ & 6.82548 & 3.54585 & 0.54836 \\
\hline $\mathrm{C}$ & 6.83836 & 2.92317 & 1.89651 \\
\hline $\mathrm{C}$ & 5.62873 & 2.44668 & 2.43995 \\
\hline $\mathrm{C}$ & 5.65425 & 1.19435 & 3.25778 \\
\hline $\mathrm{C}$ & 6.87153 & 0.50803 & 3.41537 \\
\hline $\mathrm{C}$ & 6.87924 & -0.97988 & 3.34597 \\
\hline $\mathrm{C}$ & 8.15822 & -1.65699 & 3.03209 \\
\hline $\mathrm{C}$ & 8.15486 & -2.76697 & 2.18019 \\
\hline $\mathrm{C}$ & 9.43030 & -3.20441 & 1.56803 \\
\hline $\mathrm{C}$ & 9.41335 & -3.59490 & 0.13330 \\
\hline $\mathrm{C}$ & 8.12082 & -3.53105 & -0.58427 \\
\hline $\mathrm{C}$ & 8.08923 & -2.98259 & -1.86942 \\
\hline $\mathrm{C}$ & 9.34818 & -2.50461 & -2.48340 \\
\hline $\mathrm{C}$ & 9.31250 & -1.23996 & -3.26599 \\
\hline $\mathrm{C}$ & 8.02167 & -0.52294 & -3.37635 \\
\hline $\mathrm{C}$ & 8.00655 & 0.87497 & -3.32326 \\
\hline $\mathrm{C}$ & 9.28629 & 1.60076 & -3.15517 \\
\hline $\mathrm{C}$ & 9.30614 & 2.77955 & -2.24931 \\
\hline $\mathrm{C}$ & 8.04365 & 3.16087 & -1.57697 \\
\hline $\mathrm{C}$ & 8.08241 & 3.53212 & -0.22927 \\
\hline $\mathrm{C}$ & 9.37510 & 3.50486 & 0.48809 \\
\hline $\mathrm{C}$ & 9.39841 & 2.92616 & 1.85819 \\
\hline $\mathrm{C}$ & 8.12757 & 2.40735 & 2.41596 \\
\hline $\mathrm{C}$ & 8.14315 & 1.22309 & 3.15969 \\
\hline $\mathrm{C}$ & 9.42892 & 0.52169 & 3.37296 \\
\hline $\mathrm{C}$ & 9.43629 & -0.96395 & 3.31000 \\
\hline
\end{tabular}




\begin{tabular}{|c|c|c|c|}
\hline & 10.65495 & -1.62276 & \\
\hline $\mathrm{C}$ & 10.65274 & -2.80115 & 14056 \\
\hline & 10.61709 & -3.52642 & -0.59524 \\
\hline & 10.58237 & 2.94929 & 9736 \\
\hline & 10.51716 & -0.54646 & $3.4878 \mathrm{C}$ \\
\hline C & 10.50260 & .94715 & -3.43690 \\
\hline $\mathrm{C}$ & 10.53772 & 3.17653 & -1.69169 \\
\hline C & 10.57545 & 3.54937 & -0.24486 \\
\hline$C$ & 10.62610 & 2.47341 & 37912 \\
\hline 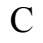 & 10.64096 & 1.21215 & 3.18083 \\
\hline & 209 & 62 & -0.26998 \\
\hline గ & 21 & 3.6 & \\
\hline 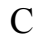 & 47 & 38 & \\
\hline $\mathrm{H}$ & 77 & 3.0 & 569 \\
\hline 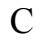 & -11 & 19 & \\
\hline H & -1 & & 993 \\
\hline & -1 & +3 & \\
\hline $\mathrm{H}$ & -12 & 6 & 269 \\
\hline 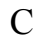 & -11 & 9 & 974 \\
\hline $\mathrm{H}$ & -12 & -3 & 611 \\
\hline$C$ & -11 & & 79 \\
\hline $\mathrm{H}$ & -12 & & \\
\hline$C$ & -11 . & 0.6 & \\
\hline $\mathbf{I}$ & -12 & & \\
\hline C & -11 & & \\
\hline$\Gamma$ & & & \\
\hline $\mathrm{C}$ & -11 & & \\
\hline I & -12 & & \\
\hline $\mathrm{C}$ & -11 & & \\
\hline J & -12 & & \\
\hline $\mathrm{C}$ & & & \\
\hline H & 12. & & \\
\hline $\mathrm{C}$ & & & \\
\hline $\mathrm{H}$ & 12 & & \\
\hline $\mathrm{C}$ & & & \\
\hline $\mathrm{H}$ & & & 839 \\
\hline $\mathrm{C}$ & & & -3.21432 \\
\hline I & & & -3.66722 \\
\hline $\mathrm{C}$ & & & \\
\hline $\mathrm{H}$ & 12.83909 & & 3.51676 \\
\hline $\mathrm{C}$ & 11.90952 & 0.87723 & 3.32413 \\
\hline $\mathrm{H}$ & 12.85048 & & 3.41518 \\
\hline $\mathrm{C}$ & 11.90279 & -3.22414 & 1.46344 \\
\hline $\mathrm{H}$ & 12.84758 & -3.19883 & 1.98905 \\
\hline $\mathrm{C}$ & 11.88637 & -3.5 & 0.17001 \\
\hline $\mathrm{H}$ & 12.81779 & -3.80584 & -0.32351 \\
\hline $\mathrm{C}$ & 11.81822 & -2.42539 & -2.60397 \\
\hline $\mathrm{H}$ & 12.76649 & -2.92031 & -2.44447 \\
\hline $\mathrm{C}$ & 11.78714 & -1.29536 & -3.31958 \\
\hline $\mathrm{H}$ & 12.71167 & -0.90539 & -3.72250 \\
\hline $\mathrm{O}$ & -1.31638 & 5.07491 & -0.04647 \\
\hline $\mathrm{N}$ & 0.05498 & 5.04551 & -0.04985 \\
\hline
\end{tabular}


Structure of LM2 within the full DFT scheme

\begin{tabular}{|c|c|c|c|}
\hline 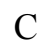 & -10.45435 & -2.99786 & 468 \\
\hline $\mathrm{C}$ & -10.46148 & -3.52038 & 0.57149 \\
\hline$C$ & -10.48273 & -2.83742 & -2.18084 \\
\hline $\mathrm{C}$ & -10.50197 & -1.72933 & -3.11603 \\
\hline $\mathrm{C}$ & -10.56764 & 1.09223 & -3.25989 \\
\hline $\mathrm{C}$ & -10.60131 & 2.29297 & -2.44670 \\
\hline $\mathrm{C}$ & -10.60835 & 3.30919 & 0.20551 \\
\hline $\mathrm{C}$ & -10.57034 & 2.97063 & 1.61616 \\
\hline $\mathrm{C}$ & -10.48575 & 0.80750 & 3.43447 \\
\hline $\mathrm{C}$ & -10.46160 & -0.64127 & 3.49943 \\
\hline C & -9.22755 & -1.29922 & 3.24283 \\
\hline $\mathrm{C}$ & -9.22638 & -2.50185 & 2.44582 \\
\hline $\mathrm{C}$ & -7.98709 & -2.93216 & 1.87057 \\
\hline $\mathrm{C}$ & -7.99379 & -3.45096 & 0.54818 \\
\hline $\mathrm{C}$ & -9.23892 & -3.51512 & -0.15707 \\
\hline $\mathrm{C}$ & -9.24864 & -3.16294 & -1.55610 \\
\hline $\mathrm{C}$ & -8.01336 & -2.74826 & -2.16078 \\
\hline $\mathrm{C}$ & -8.03102 & -1.65177 & -3.06344 \\
\hline $\mathrm{C}$ & -9.28877 & -1.01933 & -3.33964 \\
\hline $\mathrm{C}$ & -9.32235 & 0.42099 & -3.41570 \\
\hline $\mathrm{C}$ & -8.09510 & 1.13873 & -3.21883 \\
\hline $\mathrm{C}$ & -8.13095 & 2.32510 & -2.43835 \\
\hline $\mathrm{C}$ & -9.38232 & 2.74396 & -1.87088 \\
\hline $\mathrm{C}$ & -9.39010 & 3.2 & -0.52353 \\
\hline $\mathrm{C}$ & -8.14396 & 3.3 & 0.17911 \\
\hline $\mathrm{C}$ & -8.09824 & 2.99 & 1.55780 \\
\hline $\mathrm{C}$ & -9.32149 & 2.59108 & 2.18790 \\
\hline $\mathrm{C}$ & -9.27730 & 1.48497 & 3.11254 \\
\hline $\mathrm{C}$ & -8.01287 & 0.83703 & 3.33157 \\
\hline $\mathrm{C}$ & -7.99248 & -0.58313 & 3.39619 \\
\hline $\mathrm{C}$ & -6.74734 & -1.26192 & 3.19396 \\
\hline $\mathrm{C}$ & -6.74840 & -2.47266 & 2.42662 \\
\hline $\mathrm{C}$ & -5.52439 & -2.93379 & 1.86774 \\
\hline $\mathrm{C}$ & -5.53074 & -3.46509 & 0.53009 \\
\hline $\mathrm{C}$ & -6.76046 & -3.50277 & -0.18182 \\
\hline $\mathrm{C}$ & -6.76820 & -3.13334 & -1.56687 \\
\hline $\mathrm{C}$ & -5.54491 & -2.72291 & -2.17368 \\
\hline $\mathrm{C}$ & -5.55902 & -1.59595 & -3.06902 \\
\hline $\mathrm{C}$ & -6.80255 & -0.95440 & -3.33239 \\
\hline $\mathrm{C}$ & -6.8 & 0.4 & -3 \\
\hline $\mathrm{C}$ & -5.6 & 1.2 & -3.23144 \\
\hline $\mathrm{C}$ & & 2.4 & -2 \\
\hline $\mathrm{C}$ & & & \\
\hline $\mathrm{C}$ & & & -0. \\
\hline $\mathrm{C}$ & & & \\
\hline $\mathrm{C}$ & & & \\
\hline $\mathrm{C}$ & & & 043 \\
\hline $\mathrm{C}$ & -6.7 & & 3.05886 \\
\hline $\mathrm{C}$ & -5.53 & 0.89 & 3.26635 \\
\hline $\mathrm{C}$ & -5.52 & -0.54 & 3.34605 \\
\hline $\mathrm{C}$ & -4.28863 & -1.23291 & 3.14117 \\
\hline $\mathrm{C}$ & -4.29147 & -2.45778 & 2.40504 \\
\hline $\mathrm{C}$ & -3.05872 & -2.93155 & 1.84990 \\
\hline $\mathrm{C}$ & -3.06343 & -3.46259 & 0.52166 \\
\hline C & -4.30155 & -3.49453 & -0.19824 \\
\hline
\end{tabular}




\begin{tabular}{|c|c|c|c|}
\hline $\mathrm{C}$ & -4.30751 & -3.10440 & -1.57247 \\
\hline $\mathrm{C}$ & -3.07209 & -2.67953 & -2.16819 \\
\hline $\mathrm{C}$ & -3.08112 & -1.53418 & -3.02832 \\
\hline $\mathrm{C}$ & -4.33160 & -0.88617 & -3.29379 \\
\hline $\mathrm{C}$ & -4.35694 & 0.53774 & -3.36847 \\
\hline $\mathrm{C}$ & -3.12790 & 1.26501 & -3.14459 \\
\hline $\mathrm{C}$ & -3.17082 & 2.48993 & -2.42322 \\
\hline $\mathrm{C}$ & -4.44675 & 2.92016 & -1.91327 \\
\hline $\mathrm{C}$ & -4.49820 & 3.43479 & -0.58716 \\
\hline $\mathrm{C}$ & -3.27582 & 3.63002 & 0.10717 \\
\hline $\mathrm{C}$ & -3.12229 & 3.18756 & 1.47477 \\
\hline $\mathrm{C}$ & -4.33926 & 2.71519 & 2.09237 \\
\hline $\mathrm{C}$ & -4.31087 & 1.57458 & 2.95969 \\
\hline $\mathrm{C}$ & -3.05626 & 0.90686 & 3.16625 \\
\hline $\mathrm{C}$ & -3.05355 & -0.51983 & 3.28270 \\
\hline $\mathrm{C}$ & -1.82705 & -1.23158 & 3.12988 \\
\hline $\mathrm{C}$ & -1.82910 & -2.46882 & 2.39834 \\
\hline $\mathrm{C}$ & -0.59999 & -2.93701 & 1.84811 \\
\hline $\mathrm{C}$ & -0.60378 & -3.45925 & 0.51677 \\
\hline $\mathrm{C}$ & -1.83501 & -3.49960 & -0.20114 \\
\hline $\mathrm{C}$ & -1.84179 & -3.08871 & -1.57949 \\
\hline $\mathrm{C}$ & -0.61491 & -2.65301 & -2.16277 \\
\hline $\mathrm{C}$ & -0.62341 & -1.49504 & -2.99467 \\
\hline $\mathrm{C}$ & -1.85558 & -0.82544 & -3.23880 \\
\hline $\mathrm{C}$ & -1.86900 & 0.61251 & -3.27340 \\
\hline $\mathrm{C}$ & -0.64371 & 1.33468 & -3.01330 \\
\hline $\mathrm{C}$ & -0.65777 & 2.57092 & -2.28255 \\
\hline $\mathrm{C}$ & -1.96238 & 3.07325 & -1.88516 \\
\hline $\mathrm{C}$ & -2.18906 & 3.88377 & -0.74535 \\
\hline $\mathrm{C}$ & -0.52481 & 3.17989 & 1.60074 \\
\hline $\mathrm{C}$ & -1.82278 & 2.76429 & 2.03716 \\
\hline $\mathrm{C}$ & -1.82262 & 1.58584 & 2.89526 \\
\hline $\mathrm{C}$ & -0.59873 & 0.89449 & 3.20170 \\
\hline $\mathrm{C}$ & -0.60067 & -0.53648 & 3.30174 \\
\hline $\mathrm{C}$ & 0.63705 & -1.25201 & 205 \\
\hline $\mathrm{C}$ & 0.63591 & -2.46999 & 2.40262 \\
\hline $\mathrm{C}$ & 1.86873 & -2.94221 & 1.8 \\
\hline $\mathrm{C}$ & 1.86490 & -3.4 & 0.5 \\
\hline $\mathrm{C}$ & 0.63 & -3.4 & -0.2 \\
\hline $\mathrm{C}$ & 0.62499 & -3.0 & -1.57664 \\
\hline $\mathrm{C}$ & 1.84 & -2.6 & -2.17556 \\
\hline $\mathrm{C}$ & 1.84 & -1.4 & -3.02658 \\
\hline $\mathrm{C}$ & 0.60 & -0.7 & -3.21758 \\
\hline $\mathrm{C}$ & 0.6 & & -3.22838 \\
\hline $\mathrm{C}$ & 1.8 & & -3.08630 \\
\hline $\mathrm{C}$ & 1.87013 & 2.52 & -2.30354 \\
\hline $\mathrm{C}$ & 0.63407 & 3.01 & -1.70728 \\
\hline $\mathrm{C}$ & 0.79613 & 3.57 & -0.41677 \\
\hline $\mathrm{C}$ & 2.02020 & 3.53309 & 0.28023 \\
\hline $\mathrm{C}$ & 1.96601 & 3.13700 & 1.66808 \\
\hline $\mathrm{C}$ & 0.69104 & 2.70134 & 2.19536 \\
\hline $\mathrm{C}$ & 0.66080 & 1.54131 & 3.00992 \\
\hline $\mathrm{C}$ & 1.89334 & 0.85982 & 3.30509 \\
\hline $\mathrm{C}$ & 1.87885 & -0.57592 & 3.36060 \\
\hline $\mathrm{C}$ & 3.10669 & -1.28502 & 3.18861 \\
\hline $\mathrm{C}$ & 3.09981 & -2.48946 & 2.40753 \\
\hline $\mathrm{C}$ & 4.33437 & -2.94674 & 1.83682 \\
\hline
\end{tabular}




\begin{tabular}{|c|c|c|c|}
\hline $\mathrm{C}$ & 4.32786 & -3.43860 & 0.49633 \\
\hline $\mathrm{C}$ & 3.08857 & -3.47058 & -0.21992 \\
\hline $\mathrm{C}$ & 3.08086 & -3.06145 & -1.59419 \\
\hline $\mathrm{C}$ & 4.31225 & -2.64586 & -2.19643 \\
\hline $\mathrm{C}$ & 4.30805 & -1.51182 & -3.06354 \\
\hline $\mathrm{C}$ & 3.06916 & -0.81529 & -3.28194 \\
\hline $\mathrm{C}$ & 3.07411 & 0.61814 & -3.32528 \\
\hline $\mathrm{C}$ & 4.33360 & 1.29190 & -3.16503 \\
\hline $\mathrm{C}$ & 4.36520 & 2.47723 & -2.37590 \\
\hline $\mathrm{C}$ & 3.13998 & 2.95198 & -1.79362 \\
\hline $\mathrm{C}$ & 3.21179 & 3.43661 & -0.45046 \\
\hline $\mathrm{C}$ & 4.44283 & 3.42434 & 0.26888 \\
\hline $\mathrm{C}$ & 4.42568 & 3.04958 & 1.64343 \\
\hline $\mathrm{C}$ & 3.17044 & 2.68432 & 2.25403 \\
\hline $\mathrm{C}$ & 3.14082 & 1.52258 & 3.10570 \\
\hline $\mathrm{C}$ & 4.37131 & 0.82287 & 3.34786 \\
\hline $\mathrm{C}$ & 4.35363 & -0.60512 & 3.38961 \\
\hline $\mathrm{C}$ & 5.58543 & -1.31900 & 3.20552 \\
\hline $\mathrm{C}$ & 5.57405 & -2.51382 & 2.40279 \\
\hline $\mathrm{C}$ & 6.79599 & -2.96504 & 1.82756 \\
\hline $\mathrm{C}$ & 6.78709 & -3.45064 & 0.47621 \\
\hline $\mathrm{C}$ & 5.55671 & -3.47248 & -0.23435 \\
\hline $\mathrm{C}$ & 5.54887 & -3.07130 & -1.61672 \\
\hline $\mathrm{C}$ & 6.77315 & -2.67390 & -2.21884 \\
\hline $\mathrm{C}$ & 6.77277 & -1.54443 & -3.10542 \\
\hline $\mathrm{C}$ & 5.54783 & -0.85435 & -3.33872 \\
\hline $\mathrm{C}$ & 5.56180 & 0.58382 & -3.39285 \\
\hline $\mathrm{C}$ & 6.80527 & 1.25467 & -3.21114 \\
\hline $\mathrm{C}$ & 6.83568 & 2.44081 & -2.40139 \\
\hline $\mathrm{C}$ & 5.62170 & 2.90547 & -1.82520 \\
\hline $\mathrm{C}$ & 5.66112 & 3.38281 & -0.46690 \\
\hline $\mathrm{C}$ & 6.88846 & 3.37921 & 0.24260 \\
\hline $\mathrm{C}$ & 6.88369 & 2.99088 & 1.62547 \\
\hline $\mathrm{C}$ & 5.65190 & 2.62434 & 2.23900 \\
\hline $\mathrm{C}$ & 5.62716 & 1.48549 & 3.12158 \\
\hline $\mathrm{C}$ & 6.84425 & 0.79008 & 3.35784 \\
\hline $\mathrm{C}$ & 6.82406 & -0.64720 & 3.40313 \\
\hline $\mathrm{C}$ & 8.05380 & -1.35312 & 3.18640 \\
\hline $\mathrm{C}$ & 8.03983 & -2.52469 & 2.38273 \\
\hline $\mathrm{C}$ & 9.27661 & -2.98480 & 1.81410 \\
\hline $\mathrm{C}$ & 9.26620 & -3.46516 & 0.45447 \\
\hline $\mathrm{C}$ & 8.01904 & -3.46745 & -0.25470 \\
\hline $\mathrm{C}$ & 8.01219 & -3.07688 & -1.62051 \\
\hline $\mathrm{C}$ & 9.25401 & -2.70059 & -2.23217 \\
\hline $\mathrm{C}$ & 9.25603 & -1.57282 & -3.13138 \\
\hline $\mathrm{C}$ & 8.01723 & -0.87962 & -3.35662 \\
\hline $\mathrm{C}$ & 8.03373 & 0.53960 & -3.41013 \\
\hline $\mathrm{C}$ & 9.29117 & 1.21319 & -3.23545 \\
\hline $\mathrm{C}$ & 9.32027 & 2.39977 & -2.41568 \\
\hline $\mathrm{C}$ & 8.08925 & 2.84594 & -1.82825 \\
\hline $\mathrm{C}$ & 8.11664 & 3.31760 & -0.48911 \\
\hline $\mathrm{C}$ & 9.36446 & 3.32809 & 0.21803 \\
\hline $\mathrm{C}$ & 9.36435 & 2.93615 & 1.60653 \\
\hline $\mathrm{C}$ & 8.11672 & 2.55325 & 2.20793 \\
\hline $\mathrm{C}$ & 8.09670 & 1.44143 & 3.09022 \\
\hline $\mathrm{C}$ & 9.33124 & 0.75372 & 3.34480 \\
\hline $\mathrm{C}$ & 9.30941 & -0.68773 & 3.39677 \\
\hline
\end{tabular}




\begin{tabular}{|c|c|c|c|}
\hline & 59 & 33 & \\
\hline 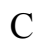 & 10.51004 & -2.59917 & 40510 \\
\hline 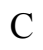 & 10.48811 & -3.54082 & -0.26897 \\
\hline$c$ & 10.48150 & 3.14543 & -1.66740 \\
\hline 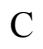 & 10.48785 & -0.93213 & .43481 \\
\hline a & 10.50561 & .51957 & -3.48982 \\
\hline $\mathrm{C}$ & 10.56075 & 2.84660 & -1.88140 \\
\hline a & 10.58507 & 3.32483 & -0.50971 \\
\hline 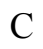 & 10.58734 & 2.55343 & 2.21991 \\
\hline C & 10.56894 & 1.42044 & 3.12937 \\
\hline C & -11.81939 & 3.26728 & -0.56453 \\
\hline & -12.76883 & 3.49254 & -0.09149 \\
\hline 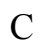 & -11.74970 & & 2.33582 \\
\hline $\mathrm{H}$ & -12.70852 & & \\
\hline $\mathrm{C}$ & -11.70815 & & \\
\hline $\mathrm{H}$ & -12.63515 & & \\
\hline C & -11.6 & & \\
\hline $\mathrm{H}$ & -12.5 & & \\
\hline 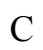 & -11.65257 & & \\
\hline $\mathrm{H}$ & -12 . & & \\
\hline 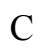 & -11.8 & & \\
\hline $\mathrm{H}$ & -12 . & & \\
\hline $\mathrm{C}$ & -11.7 & & \\
\hline $\mathrm{H}$ & -12.7 & & \\
\hline$C$ & -11.7 & & -3 \\
\hline $\mathrm{H}$ & -12.6 & & 2241 \\
\hline $\mathrm{C}$ & -11. & & \\
\hline $\mathrm{H}$ & & & 002 \\
\hline $\mathrm{C}$ & & & \\
\hline 11 & & & \\
\hline$c$ & & & \\
\hline$\Pi$ & & & \\
\hline $\mathrm{C}$ & 11. & & \\
\hline $\mathrm{H}$ & & & \\
\hline $\mathrm{C}$ & 11. & & \\
\hline $\mathrm{H}$ & 12 & & \\
\hline $\mathrm{C}$ & & & \\
\hline $\mathrm{H}$ & & & \\
\hline $\mathrm{C}$ & & & \\
\hline $\mathrm{H}$ & & & \\
\hline $\mathrm{C}$ & & & \\
\hline $\mathrm{H}$ & & & 971 \\
\hline $\mathrm{C}$ & & & 650 \\
\hline $\mathrm{H}$ & & & 097 \\
\hline $\mathrm{C}$ & 11. & & 0.50770 \\
\hline $\mathrm{H}$ & 12. & & 191 \\
\hline $\mathrm{C}$ & 11.6 & 99 & -2.36013 \\
\hline $\mathrm{H}$ & 12.6 & 10 & -2.10020 \\
\hline $\mathrm{C}$ & 11.68 & -1.69691 & -3.21996 \\
\hline $\mathrm{H}$ & 12.62870 & -1.34363 & -3.61612 \\
\hline $\mathrm{O}$ & -1.23832 & 4.73580 & -0.18073 \\
\hline $\mathrm{N}$ & -0.24221 & 3.92463 & 0.45330 \\
\hline
\end{tabular}


Structure of LM2 within the ONIOM scheme

\begin{tabular}{|c|c|c|c|}
\hline 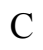 & 10.50471 & 2009 & 8846 \\
\hline $\mathrm{C}$ & 10.51394 & -3.51286 & -0.00162 \\
\hline $\mathrm{C}$ & 10.52325 & -2.40614 & 2.59790 \\
\hline $\mathrm{C}$ & 10.53193 & -1.19587 & 3.33279 \\
\hline $\mathrm{C}$ & 10.59484 & 1.59836 & 3.02303 \\
\hline $\mathrm{C}$ & 10.63358 & 2.62044 & 2.04339 \\
\hline $\mathrm{C}$ & 10.64241 & 3.19819 & -0.72878 \\
\hline $\mathrm{C}$ & 10.60512 & 2.66419 & -2.04105 \\
\hline $\mathrm{C}$ & 10.51747 & 0.25098 & -3.48367 \\
\hline $\mathrm{C}$ & 10.49845 & -1.15388 & -3.31261 \\
\hline $\mathrm{C}$ & 9.27131 & -1.78749 & -3.01811 \\
\hline $\mathrm{C}$ & 9.27815 & -2.89241 & -2.00923 \\
\hline $\mathrm{C}$ & 8.06909 & -3.21503 & -1.36744 \\
\hline $\mathrm{C}$ & 8.07815 & -3.51678 & 0.00613 \\
\hline $\mathrm{C}$ & 9.29485 & -3.47793 & 0.71150 \\
\hline $\mathrm{C}$ & 9.29729 & -2.88226 & 2.08419 \\
\hline $\mathrm{C}$ & 8.08533 & -2.36925 & 2.58894 \\
\hline $\mathrm{C}$ & 8.09238 & -1.15098 & 3.29163 \\
\hline $\mathrm{C}$ & 9.31827 & -0.48777 & 3.49687 \\
\hline $\mathrm{C}$ & 9.35195 & 0.99846 & 3.33629 \\
\hline $\mathrm{C}$ & 8.15447 & 1.65852 & 2.99565 \\
\hline $\mathrm{C}$ & 8.19747 & 2.69947 & 2.05194 \\
\hline $\mathrm{C}$ & 9.42485 & 3.03670 & 1.44607 \\
\hline $\mathrm{C}$ & 9.43182 & 3.34520 & -0.01899 \\
\hline $\mathrm{C}$ & 8.21122 & 3.29515 & -0.71585 \\
\hline $\mathrm{C}$ & 8.16813 & 2.74096 & -2.00813 \\
\hline $\mathrm{C}$ & 9.36031 & 2.26238 & -2.58145 \\
\hline $\mathrm{C}$ & 9.31008 & 0.97328 & -3.33694 \\
\hline $\mathrm{C}$ & 8.07602 & 0.29342 & -3.40919 \\
\hline $\mathrm{C}$ & 8.05985 & -1.10353 & -3.25068 \\
\hline $\mathrm{C}$ & 6.77485 & -1.76544 & -2.92093 \\
\hline $\mathrm{C}$ & 6.78339 & -2.83279 & -2.00056 \\
\hline $\mathrm{C}$ & 5.57531 & -3.23808 & -1.40248 \\
\hline $\mathrm{C}$ & 5.58508 & -3.57135 & 0.05498 \\
\hline $\mathrm{C}$ & 6.80025 & -3.44784 & 0.75506 \\
\hline $\mathrm{C}$ & 6.80119 & -2.86218 & 2.03651 \\
\hline $\mathrm{C}$ & 5.58766 & -2.39721 & 2.58274 \\
\hline $\mathrm{C}$ & 5.58883 & -1.07938 & 3.28879 \\
\hline $\mathrm{C}$ & 6.81590 & -0.39595 & 3.42048 \\
\hline $\mathrm{C}$ & 6.84459 & 1.004 & 3.27515 \\
\hline $\mathrm{C}$ & 5.64304 & 1.69654 & 2.99524 \\
\hline $\mathrm{C}$ & 5.70294 & 2.84197 & 2.04425 \\
\hline $\mathrm{C}$ & 6.93348 & 3.12 & 1.41251 \\
\hline $\mathrm{C}$ & 6.94753 & 3.42923 & 0.0 \\
\hline $\mathrm{C}$ & 5.73736 & $3.46 ?$ & -0.67317 \\
\hline $\mathrm{C}$ & 5.66449 & 2.82 & -2.02135 \\
\hline $\mathrm{C}$ & 6.85486 & 2.27 & -2.54266 \\
\hline $\mathrm{C}$ & 6.804 & 1.04 & \\
\hline $\mathrm{C}$ & 5.56 & 0.38 & \\
\hline $\mathrm{C}$ & 5.55 & -1.10 & -3.20583 \\
\hline $\mathrm{C}$ & 4.348 & -1.74 & -2.87348 \\
\hline $\mathrm{C}$ & 4.35794 & -2.83047 & -1.98029 \\
\hline $\mathrm{C}$ & 3.083 & -3.23177 & -1.34153 \\
\hline $\mathrm{C}$ & & -3.55612 & 0.02890 \\
\hline 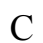 & 4.37467 & -3.46459 & 0.76628 \\
\hline
\end{tabular}




\begin{tabular}{|c|c|c|c|}
\hline $\mathrm{C}$ & 4.37440 & -2.85983 & 2.03908 \\
\hline $\mathrm{C}$ & 3.09333 & -2.33235 & 2.56679 \\
\hline $\mathrm{C}$ & 3.08966 & -1.06557 & 3.18232 \\
\hline $\mathrm{C}$ & 4.37843 & -0.35134 & 3.34474 \\
\hline $\mathrm{C}$ & 4.39833 & 1.04579 & 3.18007 \\
\hline $\mathrm{C}$ & 3.12234 & 1.74480 & 2.81919 \\
\hline $\mathrm{C}$ & 3.18784 & 2.86484 & 1.98478 \\
\hline $\mathrm{C}$ & 4.51403 & 3.23839 & 1.41180 \\
\hline $\mathrm{C}$ & 4.55293 & 3.57287 & 0.04677 \\
\hline $\mathrm{C}$ & 3.28272 & 3.59525 & -0.68433 \\
\hline $\mathrm{C}$ & 3.13746 & 2.89718 & -1.86878 \\
\hline $\mathrm{C}$ & 4.40292 & 2.33568 & -2.45824 \\
\hline $\mathrm{C}$ & 4.35821 & 1.08669 & -3.10680 \\
\hline $\mathrm{C}$ & 3.05898 & 0.36543 & -3.19490 \\
\hline $\mathrm{C}$ & 3.06606 & -1.04220 & -3.12234 \\
\hline $\mathrm{C}$ & 1.86053 & -1.73507 & -2.91297 \\
\hline $\mathrm{C}$ & 1.86565 & -2.88994 & -1.96140 \\
\hline $\mathrm{C}$ & 0.65495 & -3.23967 & -1.32912 \\
\hline $\mathrm{C}$ & 0.66929 & -3.57970 & 0.03851 \\
\hline $\mathrm{C}$ & 1.88418 & -3.52967 & 0.74850 \\
\hline $\mathrm{C}$ & 1.88274 & -2.86875 & 2.09154 \\
\hline $\mathrm{C}$ & 0.66888 & -2.32701 & 2.55810 \\
\hline $\mathrm{C}$ & 0.66258 & -1.04132 & 3.13517 \\
\hline $\mathrm{C}$ & 1.87543 & -0.35309 & 3.28720 \\
\hline $\mathrm{C}$ & 1.87815 & 1.12098 & 3.05343 \\
\hline $\mathrm{C}$ & 0.65499 & 1.77051 & 2.71182 \\
\hline $\mathrm{C}$ & 0.68154 & 2.97895 & 1.80644 \\
\hline $\mathrm{C}$ & 1.94205 & 3.38928 & 1.32984 \\
\hline $\mathrm{C}$ & 2.18429 & 4.04921 & 0.10393 \\
\hline $\mathrm{C}$ & 0.57756 & 2.90001 & -2.01531 \\
\hline $\mathrm{C}$ & 1.82315 & 2.41815 & -2.39452 \\
\hline $\mathrm{C}$ & 1.83355 & 1.0 & -3.05410 \\
\hline $\mathrm{C}$ & 0.61691 & 0.3 & -3.22077 \\
\hline $\mathrm{C}$ & 0.64228 & -1.08118 & -3.16508 \\
\hline $\mathrm{C}$ & -0.62028 & -1.82738 & -2.92449 \\
\hline $\mathrm{C}$ & -0.63236 & -2.85647 & -1.96680 \\
\hline $\mathrm{C}$ & -1.85607 & -3 & -1.39588 \\
\hline $\mathrm{C}$ & -1.87057 & -3. & 0.05410 \\
\hline $\mathrm{C}$ & -0.59792 & -3 . & 0.78981 \\
\hline $\mathrm{C}$ & -0.59971 & -2.8 & 2.03497 \\
\hline $\mathrm{C}$ & -1.87150 & -2 & 2.56063 \\
\hline $\mathrm{C}$ & -1.84123 & -0.96899 & 3.15817 \\
\hline $\mathrm{C}$ & -0.60907 & -0.27689 & 3.20922 \\
\hline $\mathrm{C}$ & -0.58251 & 1.11957 & 2.97250 \\
\hline $\mathrm{C}$ & -1.81014 & 1.79107 & 2.76482 \\
\hline $\mathrm{C}$ & -1.85118 & 2.95877 & 1.84337 \\
\hline $\mathrm{C}$ & -0.58078 & 3.31585 & 1.13476 \\
\hline $\mathrm{C}$ & -0.72466 & 3.70678 & -0.17837 \\
\hline $\mathrm{C}$ & -2.00355 & 3.55575 & -0.86782 \\
\hline $\mathrm{C}$ & -1.95382 & 2.78002 & -2.13370 \\
\hline $\mathrm{C}$ & -0.68815 & 2.26771 & -2.54095 \\
\hline $\mathrm{C}$ & -0.62331 & 1.00877 & -3.15672 \\
\hline $\mathrm{C}$ & -1.89533 & 0.27535 & -3.43668 \\
\hline $\mathrm{C}$ & -1.89234 & -1.19830 & -3.31429 \\
\hline $\mathrm{C}$ & -3.09567 & -1.86655 & -3.03961 \\
\hline $\mathrm{C}$ & -3.07706 & -2.94769 & -2.02295 \\
\hline $\mathrm{C}$ & -4.35388 & -3.29943 & -1.35358 \\
\hline
\end{tabular}




\begin{tabular}{|c|c|c|c|}
\hline$C$ & -4.34702 & -3.57041 & 117 \\
\hline 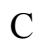 & -3.07534 & -3.46936 & 0.76413 \\
\hline $\mathrm{C}$ & -3.08097 & -2.80409 & 2.01360 \\
\hline $\mathrm{C}$ & -4.36113 & -2.24620 & 2.52706 \\
\hline $\mathrm{C}$ & -4.34427 & -0.98352 & 3.16178 \\
\hline $\mathrm{C}$ & -3.04712 & -0.25160 & 3.25434 \\
\hline $\mathrm{C}$ & -3.02640 & 1.14539 & 3.08948 \\
\hline $\mathrm{C}$ & -4.31189 & 1.85013 & 2.87677 \\
\hline $\mathrm{C}$ & -4.35459 & 2.90762 & 1.96336 \\
\hline $\mathrm{C}$ & -3.10305 & 3.29919 & 1.26594 \\
\hline $\mathrm{C}$ & -3.17282 & 3.57220 & -0.11754 \\
\hline $\mathrm{C}$ & -4.45126 & 3.39911 & -0.83462 \\
\hline $\mathrm{C}$ & -4.45793 & 2.70611 & -2.06106 \\
\hline $\mathrm{C}$ & -3.15727 & 2.24118 & -2.62607 \\
\hline $\mathrm{C}$ & -3.12135 & 0.95386 & -3.34554 \\
\hline $\mathrm{C}$ & -4.39280 & 0.20665 & -3.47275 \\
\hline $\mathrm{C}$ & -4.38239 & -1.18503 & -3.31178 \\
\hline $\mathrm{C}$ & -5.65505 & -1.89021 & -3.02890 \\
\hline $\mathrm{C}$ & -5.64051 & -2.98264 & -2.01759 \\
\hline $\mathrm{C}$ & -6.84405 & -3.33319 & -1.37795 \\
\hline $\mathrm{C}$ & -6.82938 & -3.60698 & 0.08539 \\
\hline $\mathrm{C}$ & -5.61192 & -3.51264 & 0.78155 \\
\hline $\mathrm{C}$ & -5.58410 & -2.79339 & 2.08622 \\
\hline $\mathrm{C}$ & -6.78948 & -2.30347 & 2.61145 \\
\hline $\mathrm{C}$ & -6.77481 & -0.99841 & 3.31029 \\
\hline $\mathrm{C}$ & -5.55727 & -0.29905 & 3.39368 \\
\hline $\mathrm{C}$ & -5.57450 & 1.18 & 3.28355 \\
\hline $\mathrm{C}$ & -6.8 & 1.8 & 3.0 \\
\hline $\mathrm{C}$ & -6.84920 & 2.9 & 2.0 \\
\hline $\mathrm{C}$ & -5.6 & 3.3 & 1.3 \\
\hline $\mathrm{C}$ & -5.7 & 3.5 & -0.0 \\
\hline $\mathrm{C}$ & -6. & & -0. \\
\hline $\mathrm{C}$ & -6. & & -2.0 \\
\hline $\mathrm{C}$ & -5.6 & 2.2 & -2 \\
\hline $\mathrm{C}$ & -5. & & -3 . \\
\hline $\mathrm{C}$ & -6 . & & -3 . \\
\hline $\mathrm{C}$ & & & \\
\hline $\mathrm{C}$ & -8 . & & 102 \\
\hline $\mathrm{C}$ & 133 & -2 . & -1.9 \\
\hline $\mathrm{C}$ & 287 & -3.3 & -1.34435 \\
\hline $\mathrm{C}$ & -9.38551 & -3.58088 & 0.12113 \\
\hline $\mathrm{C}$ & -8.09685 & -3.4 & 0.83271 \\
\hline $\mathrm{C}$ & -8.07559 & -2.8 & 2.07793 \\
\hline $\mathrm{C}$ & -9.34332 & -2.3 & 2.66655 \\
\hline $\mathrm{C}$ & -9.33207 & -1.0 & 3.39332 \\
\hline $\mathrm{C}$ & -8.05540 & -0.2 & 3.46884 \\
\hline $\mathrm{C}$ & -8.07121 & 1.11877 & 3.33771 \\
\hline $\mathrm{C}$ & -9.36690 & 1.79891 & 3.10126 \\
\hline $\mathrm{C}$ & -9.40751 & 2.87778 & 2.07899 \\
\hline $\mathrm{C}$ & -8.14846 & 3.21597 & 1.38225 \\
\hline $\mathrm{C}$ & -8.17931 & 3.43874 & 0.00129 \\
\hline $\mathrm{C}$ & -9.46322 & 3.33295 & -0.72185 \\
\hline $\mathrm{C}$ & -9.46319 & 2.67919 & -2.05806 \\
\hline $\mathrm{C}$ & -8.17876 & 2.15395 & -2.58167 \\
\hline $\mathrm{C}$ & -8.16956 & 0.94103 & -3.27669 \\
\hline $\mathrm{C}$ & -9.44384 & 0.20985 & -3.46076 \\
\hline $\mathrm{C}$ & -9.43049 & -1.26730 & -3.29225 \\
\hline
\end{tabular}




\begin{tabular}{|c|c|c|c|}
\hline $\mathrm{C}$ & -10.64100 & -1.92045 & \\
\hline $\mathrm{C}$ & -10.62809 & -2.99945 & -1.95561 \\
\hline$C$ & -10.59215 & -3.47451 & 0.83945 \\
\hline G & -10.56857 & -2.81586 & 2.18156 \\
\hline G & -10.55141 & -0.34385 & 3.57889 \\
\hline$C$ & -10.57001 & 1.14229 & 3.42808 \\
\hline $\mathrm{C}$ & -10.64151 & 3.18443 & 1.47186 \\
\hline $\mathrm{C}$ & -10.67138 & 3.41464 & -0.00527 \\
\hline $\mathrm{C}$ & -10.67924 & 2.18559 & -2.56764 \\
\hline $\mathrm{C}$ & -10.66779 & 0.89186 & -3.31508 \\
\hline $\mathrm{C}$ & 11.90562 & 3.25541 & \\
\hline $\mathrm{H}$ & 12.84829 & 3.45762 & \\
\hline $\mathrm{C}$ & 11.83228 & 2.15285 & -2.69771 \\
\hline $\mathrm{H}$ & 12.77846 & 2.66336 & \\
\hline $\mathrm{C}$ & 11.78875 & & \\
\hline $\mathrm{H}$ & 12.70152 & & \\
\hline $\mathrm{C}$ & & -1.9 & \\
\hline $\mathrm{H}$ & 12.67647 & & \\
\hline $\mathrm{C}$ & & & \\
\hline $\mathrm{H}$ & & & \\
\hline $\mathrm{C}$ & 11.9 & & \\
\hline $\mathrm{H}$ & & & \\
\hline $\mathrm{C}$ & & & \\
\hline $\mathrm{H}$ & & & \\
\hline $\mathrm{C}$ & & & \\
\hline $\mathrm{H}$ & & & \\
\hline $\mathrm{C}$ & & & \\
\hline $\mathrm{H}$ & & & \\
\hline $\mathrm{C}$ & & & \\
\hline $\mathrm{H}$ & & & \\
\hline ( & & & \\
\hline $\mathrm{H}$ & & & \\
\hline q & & & \\
\hline $\mathrm{H}$ & & & \\
\hline $\mathrm{C}$ & & & \\
\hline $\mathrm{H}$ & & & \\
\hline 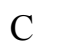 & & & \\
\hline $\mathrm{H}$ & & & \\
\hline $\mathrm{C}$ & & & \\
\hline $\mathrm{H}$ & & & -3 \\
\hline $\mathrm{C}$ & -11 & 5 & -3 \\
\hline 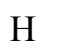 & -12 & -1 . & -3.3 \\
\hline C & -11 & -3.3 & -1.2 \\
\hline$U$ & -12 & -3. & -1.7 \\
\hline$C$ & -11.8 & -3.5 & 0.0 \\
\hline $\mathrm{H}$ & -12 & -3.7 & 0.58776 \\
\hline $\mathrm{C}$ & -11.81440 & -2.2 & 2.78868 \\
\hline $\mathrm{H}$ & -12.75232 & -2.81064 & 2.65602 \\
\hline . & -11.80523 & -1.12728 & 3.45200 \\
\hline 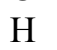 & -12.73664 & -0.73717 & 3.83906 \\
\hline . & & 4.78821 & 0.61772 \\
\hline 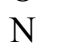 & 0.29989 & 3.92089 & -1.09799 \\
\hline
\end{tabular}


Structure of LM3 within the full DFT scheme

\begin{tabular}{|c|c|c|c|}
\hline & -1 & -2.97495 & 8177 \\
\hline C & -10.57006 & -3.29085 & 0.16401 \\
\hline C & -10.58078 & -2.15644 & -2.43168 \\
\hline C & -10.56116 & -0.90043 & -3.16187 \\
\hline C & -10.49028 & 1.91855 & -2.88262 \\
\hline $\mathrm{C}$ & -10.45229 & 3.00649 & -1.91865 \\
\hline $\mathrm{C}$ & -10.39625 & 3.62171 & 0.84654 \\
\hline $\mathrm{C}$ & -10.38774 & 3.03838 & 399 \\
\hline $\mathrm{C}$ & -10.41862 & 0.59172 & 668 \\
\hline $\mathrm{C}$ & -10.45598 & -0.85446 & 3.46066 \\
\hline $\mathrm{C}$ & -9.24932 & -1.52269 & 3.11050 \\
\hline $\mathrm{C}$ & -9.29261 & -2.60298 & 2.15637 \\
\hline $\mathrm{C}$ & -8.06904 & -2.99235 & 1.51489 \\
\hline $\mathrm{C}$ & -8.09895 & -3.30319 & 0.12806 \\
\hline $\mathrm{C}$ & -9.35125 & -3.21219 & -0.56606 \\
\hline $\mathrm{C}$ & -9.35725 & -2.63238 & -1.88671 \\
\hline $\mathrm{C}$ & -8.10928 & -2.17663 & -2.43181 \\
\hline $\mathrm{C}$ & -8.09001 & -0.94 & -3.14246 \\
\hline $\mathrm{C}$ & -9.31843 & -0.21883 & -3.28747 \\
\hline $\mathrm{C}$ & -9.28312 & 1.21540 & -3.14449 \\
\hline $\mathrm{C}$ & -8.01957 & 1.83881 & -2.86402 \\
\hline $\mathrm{C}$ & -7.98234 & 2.90570 & -1.92544 \\
\hline $\mathrm{C}$ & -9.20879 & 3.30713 & -1.29702 \\
\hline $\mathrm{C}$ & -9.18118 & 3.62 & \\
\hline $\mathrm{C}$ & -7.92719 & 3.51 & 381 \\
\hline $\mathrm{C}$ & -7.9 & 2.94335 & 2.10515 \\
\hline $\mathrm{C}$ & -9.1 & 2.50021 & 2.66527 \\
\hline $\mathrm{C}$ & -9.17 & 1.25430 & 3.38971 \\
\hline $\mathrm{C}$ & -7.948 & 0.52338 & 3.50842 \\
\hline $\mathrm{C}$ & -7.98 & -0.89242 & 3.36498 \\
\hline $\mathrm{C}$ & -6.7 & -1.58999 & 3.06794 \\
\hline $\mathrm{C}$ & -6.81354 & -2.66890 & 2.12265 \\
\hline $\mathrm{C}$ & -5.60351 & -3.07 & 1.48749 \\
\hline $\mathrm{C}$ & -5.63374 & -3.38943 & 0.08372 \\
\hline $\mathrm{C}$ & -6.87102 & -3.28316 & -0.61186 \\
\hline $\mathrm{C}$ & -6.87698 & -2.70199 & -1.92447 \\
\hline $\mathrm{C}$ & -5.64367 & -2.25411 & -2.48056 \\
\hline $\mathrm{C}$ & -5.62528 & -1.00605 & -3.19561 \\
\hline $\mathrm{C}$ & -6.83756 & -0.27333 & -3.32599 \\
\hline $\mathrm{C}$ & -6.80276 & 1.15 & -3.1 \\
\hline $\mathrm{C}$ & -5.554 & 1.78 & -2.9 \\
\hline $\mathrm{C}$ & -5.51835 & 2.86 & -1.9 \\
\hline $\mathrm{C}$ & -6.728 & 3.26 & -1.3 \\
\hline $\mathrm{C}$ & -6.70085 & 3.57 & 0.07029 \\
\hline $\mathrm{C}$ & -5.46342 & 3.48 & 0.77122 \\
\hline $\mathrm{C}$ & -5.456 & 2.910 & 2.08494 \\
\hline $\mathrm{C}$ & & 2.45 & \\
\hline $\mathrm{C}$ & -6.700 & 1.20 & \\
\hline $\mathrm{C}$ & -5.48 & 0.46 & \\
\hline $\mathrm{C}$ & & -0.96 & \\
\hline $\mathrm{C}$ & -4.30 & -1.65 & \\
\hline $\mathrm{C}$ & -4.35050 & -2.73 & \\
\hline- & & & 1.44582 \\
\hline $\mathrm{C}$ & & & \\
\hline $\mathrm{C}$ & -4.40631 & -3.34425 & -0.65359 \\
\hline
\end{tabular}




\begin{tabular}{|c|c|c|c|}
\hline $\mathrm{C}$ & -4.41255 & -2.76421 & -1.95947 \\
\hline $\mathrm{C}$ & -3.16999 & -2.31311 & -2.51764 \\
\hline $\mathrm{C}$ & -3.15278 & -1.06653 & -3.22708 \\
\hline $\mathrm{C}$ & -4.37328 & -0.32675 & -3.35151 \\
\hline $\mathrm{C}$ & -4.33855 & 1.09307 & -3.20127 \\
\hline $\mathrm{C}$ & -3.08084 & 1.72578 & -2.92337 \\
\hline $\mathrm{C}$ & -3.04609 & 2.80068 & -1.97249 \\
\hline $\mathrm{C}$ & -4.26380 & 3.20904 & -1.35399 \\
\hline $\mathrm{C}$ & -4.23527 & 3.53936 & 0.03492 \\
\hline $\mathrm{C}$ & -2.99021 & 3.48396 & 0.74347 \\
\hline $\mathrm{C}$ & -2.99229 & 2.86386 & 2.04451 \\
\hline $\mathrm{C}$ & -4.21315 & 2.39641 & 2.59261 \\
\hline $\mathrm{C}$ & -4.23493 & 1.14193 & 3.29584 \\
\hline $\mathrm{C}$ & -3.01698 & 0.40091 & 3.41357 \\
\hline $\mathrm{C}$ & -3.05304 & -1.02617 & 3.28761 \\
\hline $\mathrm{C}$ & -1.84184 & -1.72589 & 2.99234 \\
\hline $\mathrm{C}$ & -1.88168 & -2.80960 & 2.04774 \\
\hline $\mathrm{C}$ & -0.66455 & -3.20002 & 1.40565 \\
\hline $\mathrm{C}$ & -0.69215 & -3.50673 & 0.00843 \\
\hline $\mathrm{C}$ & -1.93519 & -3.41256 & -0.69030 \\
\hline $\mathrm{C}$ & -1.94296 & -2.82687 & -2.00414 \\
\hline $\mathrm{C}$ & -0.70373 & -2.36134 & -2.55067 \\
\hline $\mathrm{C}$ & -0.68745 & -1.12230 & -3.25575 \\
\hline $\mathrm{C}$ & -1.90726 & -0.38880 & -3.38783 \\
\hline $\mathrm{C}$ & -1.87555 & 1.03 & -3.22667 \\
\hline $\mathrm{C}$ & -0.62026 & 1.66 & -2.92727 \\
\hline $\mathrm{C}$ & -0.58473 & 2.71 & -1.98780 \\
\hline $\mathrm{C}$ & -1.79441 & 3.13 & -1.35345 \\
\hline $\mathrm{C}$ & -1.78941 & 3.51 & 531 \\
\hline $\mathrm{C}$ & -0.52593 & 2.73 & 1.96324 \\
\hline $\mathrm{C}$ & -1.75030 & 2.3 & 2.50058 \\
\hline $\mathrm{C}$ & -1.77050 & 1.0 & 918 \\
\hline $\mathrm{C}$ & -0.55839 & 0.3 & 3.3 \\
\hline $\mathrm{C}$ & -0.59216 & -1.0 & 3.25067 \\
\hline $\mathrm{C}$ & 0.6 & -1.7 & 942 \\
\hline $\mathrm{C}$ & 0.59089 & -2.8 & 962 \\
\hline $\mathrm{C}$ & 1.80 & -3.2 & 502 \\
\hline $\mathrm{C}$ & 1.7 & -3 & -0. \\
\hline $\mathrm{C}$ & 0.5 & -3 . & -0.72961 \\
\hline $\mathrm{C}$ & 59 & -2 . & -2.03545 \\
\hline $\mathrm{C}$ & 73 & -2 & -2.59374 \\
\hline $\mathrm{C}$ & 1.78576 & -1.17186 & 483 \\
\hline $\mathrm{C}$ & 0.56637 & -0.43763 & -3.41669 \\
\hline $\mathrm{C}$ & 0.60030 & 0.97777 & -3.25583 \\
\hline $\mathrm{C}$ & 1.84729 & 1.61866 & -2.99446 \\
\hline $\mathrm{C}$ & 1.88669 & 2.69507 & -2.03941 \\
\hline $\mathrm{C}$ & 0.66725 & 3.07144 & -1.37875 \\
\hline $\mathrm{C}$ & 0.71602 & 3.44325 & -0.02997 \\
\hline $\mathrm{C}$ & 1.94146 & 3.37513 & 0.67733 \\
\hline $\mathrm{C}$ & 1.95117 & 2.75663 & 1.98251 \\
\hline $\mathrm{C}$ & 0.69773 & 2.25428 & 2.46664 \\
\hline $\mathrm{C}$ & 0.68249 & 1.01319 & 3.18126 \\
\hline $\mathrm{C}$ & 1.90698 & 0.29336 & 3.34782 \\
\hline $\mathrm{C}$ & 1.87592 & -1.13925 & 3.21857 \\
\hline $\mathrm{C}$ & 3.08914 & -1.82182 & 2.91870 \\
\hline $\mathrm{C}$ & 3.05508 & -2.90403 & 1.97517 \\
\hline $\mathrm{C}$ & 4.27780 & -3.28801 & 1.33369 \\
\hline
\end{tabular}




\begin{tabular}{|c|c|c|c|}
\hline $\mathrm{C}$ & 4.25314 & -3.59706 & -0.06045 \\
\hline $\mathrm{C}$ & 3.00573 & -3.50768 & -0.76361 \\
\hline $\mathrm{C}$ & 2.99871 & -2.92625 & -2.07399 \\
\hline $\mathrm{C}$ & 4.23971 & -2.45994 & -2.62496 \\
\hline $\mathrm{C}$ & 4.25462 & -1.22094 & -3.33360 \\
\hline $\mathrm{C}$ & 3.02743 & -0.48808 & -3.46278 \\
\hline $\mathrm{C}$ & 3.05925 & 0.93599 & -3.30887 \\
\hline $\mathrm{C}$ & 4.31638 & 1.57295 & -3.03925 \\
\hline $\mathrm{C}$ & 4.35405 & 2.64283 & -2.09729 \\
\hline $\mathrm{C}$ & 3.13228 & 3.04527 & -1.45613 \\
\hline $\mathrm{C}$ & 3.15781 & 3.37833 & -0.06264 \\
\hline $\mathrm{C}$ & 4.40629 & 3.26001 & 0.63966 \\
\hline $\mathrm{C}$ & 4.41043 & 2.68715 & 1.94335 \\
\hline $\mathrm{C}$ & 3.15888 & 2.23727 & 2.49531 \\
\hline $\mathrm{C}$ & 3.14509 & 0.97658 & 3.19978 \\
\hline $\mathrm{C}$ & 4.36794 & 0.24544 & 3.33028 \\
\hline $\mathrm{C}$ & 4.34006 & -1.17611 & 3.18432 \\
\hline $\mathrm{C}$ & 5.56073 & -1.86549 & 2.89201 \\
\hline $\mathrm{C}$ & 5.52814 & -2.94579 & 1.94187 \\
\hline $\mathrm{C}$ & 6.74106 & -3.33005 & 1.30554 \\
\hline $\mathrm{C}$ & 6.71678 & -3.64267 & -0.09653 \\
\hline $\mathrm{C}$ & 5.48070 & -3.55536 & -0.79609 \\
\hline $\mathrm{C}$ & 5.47344 & -2.97394 & -2.11305 \\
\hline $\mathrm{C}$ & 6.70311 & -2.51267 & -2.66192 \\
\hline $\mathrm{C}$ & 6.71736 & -1.26875 & -3.37945 \\
\hline $\mathrm{C}$ & 5.50136 & -0.54111 & -3.51105 \\
\hline $\mathrm{C}$ & 5.53358 & 0.89020 & -3.36070 \\
\hline $\mathrm{C}$ & 6.77905 & 1.52099 & -3.08855 \\
\hline $\mathrm{C}$ & 6.81616 & 2.59502 & -2.13590 \\
\hline $\mathrm{C}$ & 5.60663 & 2.99409 & -1.50304 \\
\hline $\mathrm{C}$ & 5.63335 & 3.30882 & -0.09755 \\
\hline $\mathrm{C}$ & 6.86707 & 3.20365 & 0.59983 \\
\hline $\mathrm{C}$ & 6.87063 & 2.62679 & 1.91562 \\
\hline $\mathrm{C}$ & 5.63958 & 2.17985 & 2.47021 \\
\hline $\mathrm{C}$ & 5.62140 & 0.92805 & 3.18 \\
\hline $\mathrm{C}$ & 6.83244 & 0.19934 & 3.31705 \\
\hline $\mathrm{C}$ & 6.80346 & -1.23169 & 3.1 \\
\hline $\mathrm{C}$ & 8.02402 & -1.90829 & 2.8 \\
\hline $\mathrm{C}$ & 7.99270 & -2.97038 & 1.90232 \\
\hline $\mathrm{C}$ & 9.22 & -3.36838 & 1.27556 \\
\hline $\mathrm{C}$ & 9.19 & -3.67898 & -0.13278 \\
\hline $\mathrm{C}$ & 7.94 & -3.57448 & -0.82887 \\
\hline $\mathrm{C}$ & 7.93972 & -3.00509 & -2.12995 \\
\hline $\mathrm{C}$ & 9.18473 & -2.56135 & -2.69060 \\
\hline $\mathrm{C}$ & 9.19908 & -1.31364 & -3.41489 \\
\hline $\mathrm{C}$ & 7.96775 & -0.58404 & -3.53108 \\
\hline $\mathrm{C}$ & 7.99936 & 0.82752 & -3.38241 \\
\hline $\mathrm{C}$ & 9.26105 & 1.46305 & -3.12577 \\
\hline $\mathrm{C}$ & 9.29829 & 2.53837 & -2.16446 \\
\hline $\mathrm{C}$ & 8.07155 & 2.91758 & -1.52249 \\
\hline $\mathrm{C}$ & 8.09760 & 3.22537 & -0.13739 \\
\hline $\mathrm{C}$ & 9.34880 & 3.14168 & 0.56083 \\
\hline $\mathrm{C}$ & 9.35248 & 2.56383 & 1.88355 \\
\hline $\mathrm{C}$ & 8.10439 & 2.10183 & 2.42357 \\
\hline $\mathrm{C}$ & 8.08569 & 0.87400 & 3.13418 \\
\hline $\mathrm{C}$ & 9.31629 & 0.15003 & 3.28557 \\
\hline $\mathrm{C}$ & 9.28542 & -1.28480 & 3.13480 \\
\hline
\end{tabular}




\begin{tabular}{|c|c|c|c|}
\hline & 10.49427 & -1.98216 & 5918 \\
\hline $\mathrm{C}$ & 10.46205 & -3.06539 & 1.89997 \\
\hline & 10.41542 & 3.67382 & -0.86704 \\
\hline & 10.40800 & 3.09414 & 2.20005 \\
\hline & 10.43649 & -0.64583 & .62378 \\
\hline & 10.46884 & 79886 & -3.47283 \\
\hline & 10.54154 & 2.90812 & -1.58309 \\
\hline & 10.56792 & 3.22193 & -0.16533 \\
\hline 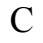 & 10.57485 & 2.08982 & 43068 \\
\hline 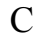 & 10.55607 & 0.83422 & 16172 \\
\hline 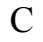 & -11.60655 & 889 & 192 \\
\hline $\mathrm{H}$ & 091 & 180 & 356 \\
\hline C & 3824 & 08 & 577 \\
\hline $\mathrm{H}$ & 2669 & 06 & 356 \\
\hline $\mathrm{C}$ & -11 . & 50 & 46 \\
\hline $\mathrm{H}$ & -12 & 53 & 131 \\
\hline 0 & -11 & 48 & 430 \\
\hline $\mathrm{H}$ & -12 & & 3.6 \\
\hline 0 & -11 . & & 036 \\
\hline $\mathrm{H}$ & -12 & & 183 \\
\hline $\mathrm{C}$ & -11 & & 222 \\
\hline $\mathrm{H}$ & -12 & & 515 \\
\hline C & -11 . & & 142 \\
\hline $\mathrm{H}$ & -12 & & 136 \\
\hline$c$ & -11 & & \\
\hline$U$ & -12 & & \\
\hline $\mathrm{C}$ & -11 & & \\
\hline 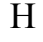 & -12 & & \\
\hline $\mathrm{C}$ & -11 & & \\
\hline 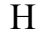 & -12 & & \\
\hline $\mathrm{C}$ & 11 & & \\
\hline $\mathrm{H}$ & 12. & & \\
\hline $\mathrm{C}$ & 11. & & \\
\hline $\mathrm{H}$ & 12. & & 162 \\
\hline $\mathrm{C}$ & 11. & & 2.30088 \\
\hline $\mathrm{H}$ & & & -2.02247 \\
\hline $\mathrm{C}$ & 11.69016 & & -3.21971 \\
\hline $\mathrm{H}$ & & & -3.64511 \\
\hline $\mathrm{C}$ & 11.74424 & & 3.36164 \\
\hline $\mathrm{H}$ & 12.70070 & & 3.48696 \\
\hline $\mathrm{C}$ & 11.71437 & & 3.21869 \\
\hline $\mathrm{H}$ & 12.64789 & & 3.23435 \\
\hline $\mathrm{C}$ & 11.64681 & -3.52388 & 1.23466 \\
\hline $\mathrm{H}$ & 12.59957 & & 1.75206 \\
\hline $\mathrm{C}$ & 11.62435 & & -0.10948 \\
\hline $\mathrm{H}$ & 12.55973 & -4.02387 & -0.61877 \\
\hline $\mathrm{C}$ & 11.60975 & -2.63516 & -2.83498 \\
\hline $\mathrm{H}$ & 12.54725 & -3.14922 & -2.65390 \\
\hline $\mathrm{C}$ & 11.62357 & -1.44588 & -3.52688 \\
\hline $\mathrm{H}$ & 12.57138 & -1.05069 & -3.87528 \\
\hline $\mathrm{O}$ & -0.47647 & 5.05029 & 1.21765 \\
\hline T & -0.51999 & 3.64885 & 0.77517 \\
\hline
\end{tabular}


Structure of LM3 within the ONIOM scheme

\begin{tabular}{|c|c|c|c|}
\hline $\mathrm{C}$ & 10.53727 & -3.12423 & 2913 \\
\hline $\mathrm{C}$ & 10.54235 & -3.48545 & -0.15939 \\
\hline $\mathrm{C}$ & 10.53607 & -2.45674 & 2.46744 \\
\hline $\mathrm{C}$ & 10.52911 & -1.26313 & 3.22988 \\
\hline $\mathrm{C}$ & 10.53032 & 1.55289 & 3.06621 \\
\hline $\mathrm{C}$ & 10.53886 & 2.64987 & 2.17034 \\
\hline $\mathrm{C}$ & 10.54357 & 3.36918 & -0.55800 \\
\hline $\mathrm{C}$ & 10.53501 & 2.85353 & -1.87734 \\
\hline $\mathrm{C}$ & 10.51985 & 0.47621 & -3.39521 \\
\hline $\mathrm{C}$ & 10.52178 & -0.93758 & -3.31208 \\
\hline $\mathrm{C}$ & 9.30121 & -1.60939 & -3.07658 \\
\hline $\mathrm{C}$ & 9.31083 & -2.77389 & -2.13739 \\
\hline $\mathrm{C}$ & 8.09988 & -3.14049 & -1.51957 \\
\hline $\mathrm{C}$ & 8.10495 & -3.50415 & -0.16128 \\
\hline $\mathrm{C}$ & 9.32022 & -3.48718 & 0.54986 \\
\hline $\mathrm{C}$ & 9.31608 & -2.93723 & 1.94127 \\
\hline $\mathrm{C}$ & 8.09796 & -2.46008 & 2.46350 \\
\hline $\mathrm{C}$ & 8.09035 & -1.26790 & 3.20938 \\
\hline $\mathrm{C}$ & 9.30368 & -0.58730 & 3.42948 \\
\hline $\mathrm{C}$ & 9.30397 & 0.90590 & 3.34229 \\
\hline $\mathrm{C}$ & 8.09077 & 1.55722 & 3.04410 \\
\hline $\mathrm{C}$ & 8.10058 & 2.65717 & 2.16782 \\
\hline $\mathrm{C}$ & 9.31932 & 3.06899 & 1.59353 \\
\hline $\mathrm{C}$ & 9.32302 & 3.45553 & 0.14796 \\
\hline $\mathrm{C}$ & 8.10700 & 3.39282 & -0.55867 \\
\hline $\mathrm{C}$ & 8.09725 & 2.86759 & -1.86340 \\
\hline $\mathrm{C}$ & 9.30724 & 2.43314 & -2.43756 \\
\hline $\mathrm{C}$ & 9.29763 & 1.16835 & -3.23614 \\
\hline $\mathrm{C}$ & 8.08011 & 0.47049 & -3.36491 \\
\hline $\mathrm{C}$ & 8.08292 & -0.93363 & -3.28676 \\
\hline $\mathrm{C}$ & 6.80305 & -1.63419 & -3.01344 \\
\hline $\mathrm{C}$ & 6.81278 & -2.74042 & -2.14091 \\
\hline $\mathrm{C}$ & 5.60185 & -3.17764 & -1.56611 \\
\hline $\mathrm{C}$ & 5.60756 & -3.56934 & -0.12351 \\
\hline $\mathrm{C}$ & 6.82247 & -3.47405 & 0.58507 \\
\hline $\mathrm{C}$ & 6.81839 & -2.94875 & 1.89223 \\
\hline $\mathrm{C}$ & 5.59992 & -2.52734 & 2.46195 \\
\hline $\mathrm{C}$ & 5.58968 & -1.25013 & 3.23849 \\
\hline $\mathrm{C}$ & 6.80348 & -0.54634 & 3.38260 \\
\hline $\mathrm{C}$ & 6.80257 & 0.86018 & 3.29695 \\
\hline $\mathrm{C}$ & 5.58745 & 1.538 & 3.06377 \\
\hline $\mathrm{C}$ & 5.60269 & 2.728 & 2.15736 \\
\hline $\mathrm{C}$ & 6.82229 & 3.08285 & 1.54631 \\
\hline $\mathrm{C}$ & 6.82741 & 3.45968 & 0.18847 \\
\hline $\mathrm{C}$ & 5.61266 & 3.48159 & -0.52474 \\
\hline $\mathrm{C}$ & 5.59914 & 2.89546 & -1.90426 \\
\hline $\mathrm{C}$ & 6.80786 & 2.39 & -2.42721 \\
\hline $\mathrm{C}$ & 6.79 & 1.19 & -3.16534 \\
\hline $\mathrm{C}$ & 5.57 & 0.50 & -3.35900 \\
\hline $\mathrm{C}$ & 5.58 & -0.98 & -3.28937 \\
\hline $\mathrm{C}$ & 4.37309 & -1.64678 & -3.00061 \\
\hline $\mathrm{C}$ & 4.38228 & -2.75883 & -2.13577 \\
\hline $\mathrm{C}$ & 3.10249 & -3.18353 & -1.51329 \\
\hline $\mathrm{C}$ & 3.10941 & -3.55818 & -0.15514 \\
\hline $\mathrm{C}$ & 4.39271 & -3.50044 & 0.58809 \\
\hline
\end{tabular}




\begin{tabular}{|c|c|c|c|}
\hline $\mathrm{C}$ & 4.38856 & -2.97134 & 1.89408 \\
\hline $\mathrm{C}$ & 3.10150 & -2.49579 & 2.45946 \\
\hline $\mathrm{C}$ & 3.08994 & -1.28192 & 3.17302 \\
\hline $\mathrm{C}$ & 4.37016 & -0.55131 & 3.35393 \\
\hline $\mathrm{C}$ & 4.36695 & 0.85336 & 3.25338 \\
\hline $\mathrm{C}$ & 3.08297 & 1.54190 & 2.95899 \\
\hline $\mathrm{C}$ & 3.10915 & 2.68012 & 2.13256 \\
\hline $\mathrm{C}$ & 4.39490 & 3.11764 & 1.54589 \\
\hline $\mathrm{C}$ & 4.39905 & 3.51899 & 0.19422 \\
\hline $\mathrm{C}$ & 3.11889 & 3.46886 & -0.56406 \\
\hline $\mathrm{C}$ & 3.10725 & 2.88920 & -1.80697 \\
\hline $\mathrm{C}$ & 4.37622 & 2.40116 & -2.40567 \\
\hline $\mathrm{C}$ & 4.36049 & 1.18671 & -3.12327 \\
\hline $\mathrm{C}$ & 3.07278 & 0.45202 & -3.27756 \\
\hline $\mathrm{C}$ & 3.08526 & -0.95552 & -3.24761 \\
\hline $\mathrm{C}$ & 1.87644 & -1.65574 & -3.05598 \\
\hline $\mathrm{C}$ & 1.88209 & -2.82795 & -2.12541 \\
\hline $\mathrm{C}$ & 0.66760 & -3.19611 & -1.50412 \\
\hline $\mathrm{C}$ & 0.68059 & -3.58779 & -0.14998 \\
\hline $\mathrm{C}$ & 1.89674 & -3.57152 & 0.56231 \\
\hline $\mathrm{C}$ & 1.89137 & -3.00520 & 1.94790 \\
\hline $\mathrm{C}$ & 0.67071 & -2.51502 & 2.45934 \\
\hline $\mathrm{C}$ & 0.65630 & -1.28912 & 3.15538 \\
\hline $\mathrm{C}$ & 1.86844 & -0.6 & 3.35409 \\
\hline $\mathrm{C}$ & 1.85752 & 0.8 & 3.22628 \\
\hline $\mathrm{C}$ & 0.62664 & 1.53380 & 2.96176 \\
\hline $\mathrm{C}$ & 0.63972 & 2.74558 & 2.09292 \\
\hline $\mathrm{C}$ & 1.84637 & 3.12189 & 1.49043 \\
\hline $\mathrm{C}$ & 1.89197 & 3.56534 & 0.17333 \\
\hline $\mathrm{C}$ & 0.66672 & 2.84030 & -1.81366 \\
\hline $\mathrm{C}$ & 1.84270 & 2.40 & -2.33289 \\
\hline $\mathrm{C}$ & 1.84816 & 1. & -3.10093 \\
\hline $\mathrm{C}$ & 0.63319 & 0.4 & -3.26557 \\
\hline $\mathrm{C}$ & 0.65479 & 867 & -3.27848 \\
\hline $\mathrm{C}$ & -0.61049 & 672 & -3.04765 \\
\hline $\mathrm{C}$ & -0.62291 & -2. & -2.12672 \\
\hline $\mathrm{C}$ & -1.84999 & -3 . & -1.57129 \\
\hline $\mathrm{C}$ & -1.86621 & -3 & -0.14079 \\
\hline $\mathrm{C}$ & -0.5 & -3 & 0.60218 \\
\hline $\mathrm{C}$ & -0.59900 & -3.01563 & 1.89092 \\
\hline $\mathrm{C}$ & -1.87971 & -2.53421 & 2.45359 \\
\hline $\mathrm{C}$ & -1.85805 & -1.22568 & 3.16515 \\
\hline $\mathrm{C}$ & -0.62536 & -0.54257 & 3.29421 \\
\hline $\mathrm{C}$ & -0.60345 & 0.86612 & 3.16911 \\
\hline $\mathrm{C}$ & -1.82309 & 1.55143 & 2.95981 \\
\hline $\mathrm{C}$ & -1.83741 & 2.76274 & 2.09035 \\
\hline $\mathrm{C}$ & -0.56017 & 3.13564 & 1.44697 \\
\hline $\mathrm{C}$ & -0.57488 & 3.58116 & 0.15226 \\
\hline $\mathrm{C}$ & -1.85734 & 3.55397 & -0.59329 \\
\hline $\mathrm{C}$ & -1.84634 & 2.84518 & -1.90030 \\
\hline $\mathrm{C}$ & -0.61946 & 2.31826 & -2.35367 \\
\hline $\mathrm{C}$ & -0.59038 & 1.11515 & -3.09217 \\
\hline $\mathrm{C}$ & -1.86519 & 0.42718 & -3.39684 \\
\hline $\mathrm{C}$ & -1.87780 & -1.05324 & -3.38152 \\
\hline $\mathrm{C}$ & -3.08406 & -1.71711 & -3.13302 \\
\hline $\mathrm{C}$ & -3.07070 & -2.85438 & -2.17997 \\
\hline $\mathrm{C}$ & -4.35134 & -3.23490 & -1.53512 \\
\hline
\end{tabular}




\begin{tabular}{|c|c|c|c|}
\hline $\mathrm{C}$ & -4.34925 & -3.59209 & -0.18037 \\
\hline$C$ & -3.07682 & -3.53817 & 0.57283 \\
\hline $\mathrm{C}$ & -3.08919 & -2.96386 & 1.86664 \\
\hline $\mathrm{C}$ & -4.37652 & -2.44162 & 2.40926 \\
\hline $\mathrm{C}$ & -4.36367 & -1.22302 & 3.12398 \\
\hline $\mathrm{C}$ & -3.06592 & -0.50990 & 3.28759 \\
\hline $\mathrm{C}$ & -3.04068 & 0.89426 & 3.20691 \\
\hline $\mathrm{C}$ & -4.30910 & 1.62178 & 2.99312 \\
\hline $\mathrm{C}$ & -4.31915 & 2.73525 & 2.14285 \\
\hline $\mathrm{C}$ & -3.05044 & 3.16041 & 1.49867 \\
\hline $\mathrm{C}$ & -3.06454 & 3.55324 & 0.13866 \\
\hline $\mathrm{C}$ & -4.34864 & 3.46459 & -0.60271 \\
\hline $\mathrm{C}$ & -4.36148 & 2.83435 & -1.86034 \\
\hline $\mathrm{C}$ & -3.06731 & 2.36659 & -2.43081 \\
\hline $\mathrm{C}$ & -3.06783 & 1.12259 & -3.23440 \\
\hline $\mathrm{C}$ & -4.34609 & 0.40653 & -3.40503 \\
\hline $\mathrm{C}$ & -4.35748 & -0.99408 & -3.34482 \\
\hline $\mathrm{C}$ & -5.63591 & -1.69685 & -3.10916 \\
\hline $\mathrm{C}$ & -5.63410 & -2.85993 & -2.17978 \\
\hline $\mathrm{C}$ & -6.84329 & -3.24282 & -1.57250 \\
\hline $\mathrm{C}$ & -6.83682 & -3.62485 & -0.13380 \\
\hline $\mathrm{C}$ & -5.62099 & -3.58314 & 0.57461 \\
\hline $\mathrm{C}$ & -5.59996 & -2.95268 & 1.92399 \\
\hline $\mathrm{C}$ & -6.80951 & -2.48768 & 2.46778 \\
\hline $\mathrm{C}$ & -6.79602 & -1.22033 & 3.23245 \\
\hline $\mathrm{C}$ & -5.57538 & -0.5 & 187 \\
\hline $\mathrm{C}$ & -5.57999 & 0.9 & 53 \\
\hline $\mathrm{C}$ & -6.79 & 1.6 & 75 \\
\hline $\mathrm{C}$ & -6.8 & 2.7 & 587 \\
\hline $\mathrm{C}$ & -5.6 & 3.2 & 1.5 \\
\hline $\mathrm{C}$ & -5.6 & 3.5 & 67 \\
\hline $\mathrm{C}$ & -6.8 & 3.5 & 370 \\
\hline $\mathrm{C}$ & -6.8 & 2.8 & 034 \\
\hline $\mathrm{C}$ & -5.58923 & 2.39892 & 780 \\
\hline $\mathrm{C}$ & -5.6 & 1.1 & 583 \\
\hline $\mathrm{C}$ & -6.8 & & 382 \\
\hline $\mathrm{C}$ & -6.8 & -1 . & 889 \\
\hline $\mathrm{C}$ & -8 . & -1 . & 373 \\
\hline $\mathrm{C}$ & -8 . & -2 . & 295 \\
\hline $\mathrm{C}$ & -9 & & \\
\hline $\mathrm{C}$ & -9.39567 & -3.58533 & 145 \\
\hline $\mathrm{C}$ & -8.11012 & -3.50354 & 0.61489 \\
\hline $\mathrm{C}$ & -8.09502 & -2.94736 & 1.89721 \\
\hline $\mathrm{C}$ & -9.36547 & -2.48543 & 2.49986 \\
\hline $\mathrm{C}$ & -9.35308 & -1.21897 & 3.27974 \\
\hline $\mathrm{C}$ & -8.07146 & -0.48812 & 3.40553 \\
\hline $\mathrm{C}$ & -8.07198 & 0.90910 & 3.34458 \\
\hline $\mathrm{C}$ & -9.35506 & 1.61837 & 3.13463 \\
\hline $\mathrm{C}$ & -9.36613 & 2.77846 & 2.20424 \\
\hline $\mathrm{C}$ & -8.09281 & 3.15817 & 1.55316 \\
\hline $\mathrm{C}$ & -8.10094 & 3.50453 & 0.19798 \\
\hline $\mathrm{C}$ & -9.37880 & 3.47482 & -0.54408 \\
\hline $\mathrm{C}$ & -9.37512 & 2.89754 & -1.91450 \\
\hline $\mathrm{C}$ & -8.09409 & 2.37421 & -2.44690 \\
\hline $\mathrm{C}$ & -8.10137 & 1.18970 & -3.18953 \\
\hline $\mathrm{C}$ & -9.38523 & 0.48676 & -3.41592 \\
\hline $\mathrm{C}$ & -9.39525 & -0.99795 & -3.33127 \\
\hline
\end{tabular}




\begin{tabular}{|c|c|c|c|}
\hline & -10.61573 & -1.65265 & \\
\hline $\mathrm{C}$ & -10.62040 & -2.81659 & -2.14128 \\
\hline & -10.60701 & -3.52297 & 0.60158 \\
\hline & -10.58997 & -2.94653 & 1.98042 \\
\hline C & -10.56804 & -0.53606 & 3.47890 \\
\hline C & -10.56992 & 0.95635 & 3.40010 \\
\hline $\mathrm{C}$ & -10.58769 & 3.15641 & 1.61378 \\
\hline $\mathrm{C}$ & -10.59419 & 3.52313 & 0.16467 \\
\hline $\mathrm{C}$ & -10.59434 & 2.44768 & -2.45839 \\
\hline $\mathrm{C}$ & -10.59929 & 1.17955 & -3.24848 \\
\hline C & 11.79841 & 3.44398 & 0.23101 \\
\hline $\mathrm{H}$ & 12.73386 & 3.71273 & -0.24072 \\
\hline C & 11.78267 & 2.37994 & \\
\hline $\mathrm{H}$ & 12.71749 & 2.90282 & -2.37552 \\
\hline $\mathrm{C}$ & 11.77488 & 1.25517 & -3.24792 \\
\hline $\mathrm{H}$ & 12.70400 & & \\
\hline C & 11.7 & -1.6 & \\
\hline $\mathrm{H}$ & 12.70 & -1.3 & 2670 \\
\hline $\mathrm{C}$ & 11.78600 & -2.7 & \\
\hline 11 & & -3.2 & \\
\hline 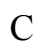 & 11.7 & & \\
\hline $\mathrm{H}$ & 12.7 & 3.1 & \\
\hline 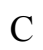 & 11.7 & 0.8 & \\
\hline $\mathrm{H}$ & & & \\
\hline $\mathrm{C}$ & 11.7 & -0.5 & \\
\hline $\mathrm{H}$ & & & \\
\hline $\mathrm{C}$ & & & \\
\hline $\mathrm{H}$ & & & \\
\hline $\mathrm{C}$ & & & \\
\hline $\mathrm{H}$ & & & \\
\hline & & & \\
\hline $\mathrm{H}$ & & & \\
\hline & & & \\
\hline $\mathrm{H}$ & & & \\
\hline$C$ & & & \\
\hline $\mathrm{I}$ & & & \\
\hline$C$ & & & \\
\hline $\mathrm{H}$ & & & \\
\hline C & & & -3.4 \\
\hline F & -12 & 0.9 & -3 \\
\hline C & -11 & -0.9 & -3 \\
\hline $\mathrm{H}$ & -12 & -1.4 & -3. \\
\hline $\mathrm{C}$ & -11 . & -3.2 & -1.4 \\
\hline $\mathrm{H}$ & -12 & -3.2 & -2.0 \\
\hline 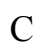 & -11.8 & -3.5 & -0.1 \\
\hline $\mathrm{H}$ & -12. & -3.8 & 0.31011 \\
\hline 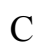 & -11.83739 & -2.43737 & 2.59994 \\
\hline- & -12.77728 & -2.94723 & 2.43798 \\
\hline $\mathrm{C}$ & -11.82692 & -1.30202 & 3.30734 \\
\hline 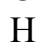 & -12.75891 & -0.92113 & 3.70181 \\
\hline $\mathrm{O}$ & & 5.11795 & 0.9767 \\
\hline $\mathrm{N}$ & 0.65136 & 3.75937 & -0.63208 \\
\hline
\end{tabular}


Structure of $\mathrm{C}_{199} \mathrm{H}_{20}$ within the Full DFT scheme

\begin{tabular}{|c|c|c|c|}
\hline $\mathrm{C}$ & -1.97155 & 3.94199 & -0.50099 \\
\hline $\mathrm{C}$ & -3.10126 & 3.71637 & 0.28510 \\
\hline $\mathrm{C}$ & -2.97849 & 2.97711 & 1.49622 \\
\hline $\mathrm{C}$ & -0.34404 & 2.75777 & 1.40892 \\
\hline $\mathrm{C}$ & -1.67959 & 2.40653 & 1.87054 \\
\hline $\mathrm{C}$ & -0.68492 & 2.57492 & -2.01082 \\
\hline $\mathrm{C}$ & -1.92639 & 3.16420 & -1.67372 \\
\hline $\mathrm{C}$ & 0.49257 & 2.88750 & -1.23298 \\
\hline $\mathrm{C}$ & 0.49363 & 3.24872 & 0.16716 \\
\hline $\mathrm{C}$ & -5.56769 & 3.58847 & 0.35370 \\
\hline $\mathrm{C}$ & -5.48340 & 3.04479 & 1.68035 \\
\hline $\mathrm{C}$ & -3.13275 & 1.48696 & -3.03751 \\
\hline $\mathrm{C}$ & -3.14266 & 2.68261 & -2.26984 \\
\hline $\mathrm{C}$ & -4.38514 & 3.17130 & -1.74732 \\
\hline $\mathrm{C}$ & -4.37580 & 3.68017 & -0.40679 \\
\hline $\mathrm{C}$ & -4.20831 & 2.53464 & 2.12088 \\
\hline $\mathrm{C}$ & -4.22206 & 1.33471 & 2.89268 \\
\hline $\mathrm{C}$ & -2.99390 & 0.62526 & 3.05980 \\
\hline $\mathrm{C}$ & -1.89491 & 0.80087 & -3.21905 \\
\hline $\mathrm{C}$ & -0.67115 & 1.45590 & -2.88872 \\
\hline $\mathrm{C}$ & -1.74919 & 1.26887 & 2.78820 \\
\hline $\mathrm{C}$ & -0.57919 & 0.55101 & 3.17861 \\
\hline $\mathrm{C}$ & 0.56453 & 0.79373 & -3.12662 \\
\hline $\mathrm{C}$ & 1.76929 & 1.49730 & -2.84869 \\
\hline $\mathrm{C}$ & 1.74642 & 2.59751 & -1.90988 \\
\hline $\mathrm{C}$ & 1.83309 & 3.48909 & 0.68737 \\
\hline $\mathrm{C}$ & 1.93177 & 2.96705 & 2.01225 \\
\hline $\mathrm{C}$ & 0.68483 & 2.41039 & 2.35777 \\
\hline $\mathrm{C}$ & 0.65452 & 1.23128 & 3.13303 \\
\hline $\mathrm{C}$ & 2.99696 & 3.06312 & -1.41401 \\
\hline $\mathrm{C}$ & 3.03049 & 3.53178 & -0.06460 \\
\hline $\mathrm{C}$ & -5.60554 & 1.49088 & -3.08916 \\
\hline $\mathrm{C}$ & -5.61099 & 2.69337 & -2.29473 \\
\hline $\mathrm{C}$ & -6.83894 & 3.10040 & -1.69122 \\
\hline $\mathrm{C}$ & -6.81585 & 3.54455 & -0.33697 \\
\hline $\mathrm{C}$ & -6.69799 & 2.58423 & 2.28206 \\
\hline $\mathrm{C}$ & -6.68499 & 1.38497 & 3.05453 \\
\hline $\mathrm{C}$ & -5.45296 & 0.68095 & 3.20062 \\
\hline $\mathrm{C}$ & -5.48003 & -0.75729 & 3.23273 \\
\hline $\mathrm{C}$ & -4.27106 & -1.47383 & 3.00414 \\
\hline $\mathrm{C}$ & -8.02892 & 3.50170 & 0.42212 \\
\hline $\mathrm{C}$ & -7.96897 & 3.02828 & 1.75950 \\
\hline $\mathrm{C}$ & -3.12306 & -1.32844 & -3.11664 \\
\hline $\mathrm{C}$ & -4.36520 & -0.62992 & -3.31051 \\
\hline $\mathrm{C}$ & -4.37011 & 0.79922 & -3.28332 \\
\hline $\mathrm{C}$ & -3.02317 & -0.79813 & 3.16749 \\
\hline $\mathrm{C}$ & -1.81478 & -1.53901 & 3.00557 \\
\hline $\mathrm{C}$ & -0.65697 & -1.32937 & -3.12109 \\
\hline $\mathrm{C}$ & -1.88972 & -0.63339 & -3.30432 \\
\hline $\mathrm{C}$ & -0.58898 & -0.87354 & 3.23432 \\
\hline $\mathrm{C}$ & 0.65319 & -1.56683 & 3.04979 \\
\hline $\mathrm{C}$ & 1.81728 & -1.30163 & -3.13950 \\
\hline $\mathrm{C}$ & 0.57750 & -0.61780 & -3.27988 \\
\hline $\mathrm{C}$ & 1.88784 & 0.55042 & 3.41248 \\
\hline $\mathrm{C}$ & 1.88520 & -0.88958 & 3.31912 \\
\hline
\end{tabular}




\begin{tabular}{|c|c|c|c|}
\hline 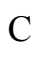 & 3.02615 & -0.56703 & -3.29818 \\
\hline$c$ & 3.00396 & 0.86237 & -3.16204 \\
\hline $\mathrm{C}$ & 4.23488 & 1.55584 & -2.92890 \\
\hline $\mathrm{C}$ & 4.23010 & 2.67758 & -2.04969 \\
\hline $\mathrm{C}$ & 4.29942 & 3.49082 & 0.63579 \\
\hline $\mathrm{C}$ & 4.35133 & 2.96205 & 1.96309 \\
\hline $\mathrm{C}$ & 3.13202 & 2.50085 & 2.55835 \\
\hline $\mathrm{C}$ & 3.12109 & 1.25020 & 3.27590 \\
\hline $\mathrm{C}$ & 5.48164 & 3.11933 & -1.50313 \\
\hline $\mathrm{C}$ & 5.51691 & 3.52056 & -0.11808 \\
\hline $\mathrm{C}$ & -10.47883 & -2.99214 & 1.74566 \\
\hline $\mathrm{C}$ & -10.50745 & -3.43917 & 0.37186 \\
\hline $\mathrm{C}$ & -10.53654 & -2.53717 & -2.30720 \\
\hline $\mathrm{C}$ & -10.54208 & -1.34777 & -3.12640 \\
\hline $\mathrm{C}$ & -10.55310 & 1.48164 & -3.06709 \\
\hline $\mathrm{C}$ & -10.55177 & 2.63848 & -2.20326 \\
\hline $\mathrm{C}$ & -10.49306 & 3.51129 & 0.49099 \\
\hline $\mathrm{C}$ & -10.43975 & 3.05720 & 1.86016 \\
\hline $\mathrm{C}$ & -10.39296 & 0.73368 & 3.46601 \\
\hline $\mathrm{C}$ & -10.41149 & -0.70942 & 3.42522 \\
\hline $\mathrm{C}$ & -9.19834 & -1.39187 & 3.12929 \\
\hline $\mathrm{C}$ & -9.23388 & -2.56200 & 2.28414 \\
\hline $\mathrm{C}$ & -8.01239 & -3.00041 & 1.67818 \\
\hline $\mathrm{C}$ & -8.03885 & -3.43914 & 0.32847 \\
\hline $\mathrm{C}$ & -9.28987 & -3.42368 & -0.36713 \\
\hline $\mathrm{C}$ & -9.30355 & -2.95224 & -1.73212 \\
\hline $\mathrm{C}$ & -8.06603 & -2.50457 & -2.30187 \\
\hline $\mathrm{C}$ & $-8.0^{\prime}$ & -1.32578 & 9316 \\
\hline $\mathrm{C}$ & -9.3 & -0.64707 & 666 \\
\hline $\mathrm{C}$ & -9.3 & 40 & 034 \\
\hline $\mathrm{C}$ & -8.0 & 79 & 523 \\
\hline $\mathrm{C}$ & -8.0 & & 368 \\
\hline $\mathrm{C}$ & -9.3 & & 13 \\
\hline $\mathrm{C}$ & -9.2 & & 60 \\
\hline $\mathrm{C}$ & -9.1 & & \\
\hline $\mathrm{C}$ & -9. & & \\
\hline $\mathrm{C}$ & & & \\
\hline $\mathrm{C}$ & & & 23 \\
\hline $\mathrm{C}$ & & & \\
\hline $\mathrm{C}$ & & & 2.24460 \\
\hline $\mathrm{C}$ & -5.5 & -3.0 & 1.65911 \\
\hline $\mathrm{C}$ & -5.56 & -3.5 & 0.28657 \\
\hline $\mathrm{C}$ & -6.80 & -3.4 & -0.41425 \\
\hline $\mathrm{C}$ & -6.81 & -2.9 & -1.75473 \\
\hline $\mathrm{C}$ & -5.5 & -2.53313 & -2.33647 \\
\hline $\mathrm{C}$ & -5.59 & -1.32632 & -3.12283 \\
\hline $\mathrm{C}$ & -6.83 & -0.6 & -3.31005 \\
\hline $\mathrm{C}$ & -6.83549 & 0.79570 & -3.28942 \\
\hline $\mathrm{C}$ & -4.29797 & -2.66560 & 2.21534 \\
\hline $\mathrm{C}$ & -3.07048 & -3.12593 & 1.62310 \\
\hline $\mathrm{C}$ & -3.08835 & -3.54220 & 0.25783 \\
\hline $\mathrm{C}$ & -4.34021 & -3.50197 & -0.45045 \\
\hline $\mathrm{C}$ & -4.35362 & -2.99034 & -1.78455 \\
\hline $\mathrm{C}$ & -3.11405 & -2.53807 & -2.35445 \\
\hline $\mathrm{C}$ & -1.82874 & -2.72463 & 2.19987 \\
\hline $\mathrm{C}$ & -0.59623 & -3.14077 & 1.60934 \\
\hline $\mathrm{C}$ & -0.61190 & -3.54985 & 0.23750 \\
\hline
\end{tabular}




\begin{tabular}{|c|c|c|c|}
\hline $\mathrm{C}$ & -1.85635 & -3.52990 & -0.47230 \\
\hline $\mathrm{C}$ & -1.87372 & -3.00887 & -1.81334 \\
\hline $\mathrm{C}$ & -0.64351 & -2.54659 & -2.37392 \\
\hline $\mathrm{C}$ & 0.64791 & -2.71324 & 2.19454 \\
\hline $\mathrm{C}$ & 1.88313 & -3.12173 & 1.60109 \\
\hline $\mathrm{C}$ & 1.87043 & -3.53058 & 0.21878 \\
\hline $\mathrm{C}$ & 0.62617 & -3.50955 & -0.49290 \\
\hline $\mathrm{C}$ & 0.60820 & -3.00019 & -1.82737 \\
\hline $\mathrm{C}$ & 1.83578 & -2.53321 & -2.39631 \\
\hline $\mathrm{C}$ & 3.11820 & -1.56106 & 3.06573 \\
\hline $\mathrm{C}$ & 3.11508 & -2.69417 & 2.18237 \\
\hline $\mathrm{C}$ & 4.35382 & -3.08593 & 1.57113 \\
\hline $\mathrm{C}$ & 4.34344 & -3.48318 & 0.20038 \\
\hline $\mathrm{C}$ & 3.09563 & -3.47976 & -0.51033 \\
\hline $\mathrm{C}$ & 3.07669 & -2.96696 & -1.84919 \\
\hline $\mathrm{C}$ & 4.30232 & -2.46954 & -2.40918 \\
\hline $\mathrm{C}$ & 4.27654 & -1.24837 & -3.14740 \\
\hline $\mathrm{C}$ & 4.35920 & 0.54472 & 3.41708 \\
\hline $\mathrm{C}$ & 4.35902 & -0.88022 & 3.31492 \\
\hline $\mathrm{C}$ & 5.59735 & -1.55364 & 3.05760 \\
\hline $\mathrm{C}$ & 5.59408 & -2.68205 & 2.16360 \\
\hline $\mathrm{C}$ & 6.81962 & -3.06967 & 1.55169 \\
\hline $\mathrm{C}$ & 6.80834 & -3.45411 & 0.16822 \\
\hline $\mathrm{C}$ & 5.57242 & -3.44265 & -0.53499 \\
\hline $\mathrm{C}$ & 5.55085 & -2.91788 & -1.87592 \\
\hline $\mathrm{C}$ & 6.76289 & -2.42674 & -2.43605 \\
\hline $\mathrm{C}$ & 6.73424 & -1.20565 & -3.19031 \\
\hline $\mathrm{C}$ & 5.49496 & -0.52669 & -3.34292 \\
\hline $\mathrm{C}$ & 5.47368 & 0.90966 & -3.24260 \\
\hline $\mathrm{C}$ & 6.69658 & 1.59751 & -3.01726 \\
\hline $\mathrm{C}$ & 6.69989 & 2.72477 & -2.12717 \\
\hline $\mathrm{C}$ & 6.76343 & 3.46952 & 0.57011 \\
\hline $\mathrm{C}$ & 6.80134 & 2.93744 & 1.90315 \\
\hline $\mathrm{C}$ & 5.59026 & 2.48544 & 2.48756 \\
\hline $\mathrm{C}$ & 5.59570 & 1.25339 & 3.24028 \\
\hline $\mathrm{C}$ & 6.82408 & 0.55672 & 3.3 \\
\hline $\mathrm{C}$ & 6.82623 & -0.87810 & 3.30232 \\
\hline $\mathrm{C}$ & 8.06533 & -1.54044 & 740 \\
\hline $\mathrm{C}$ & 8.06212 & -2.6 & 074 \\
\hline $\mathrm{C}$ & 9.30299 & -3.0 & 559 \\
\hline $\mathrm{C}$ & 9.29060 & -3.41650 & 0.13087 \\
\hline $\mathrm{C}$ & 8.03779 & -3.37817 & -0.56717 \\
\hline $\mathrm{C}$ & 8.01483 & -2.85488 & -1.88672 \\
\hline $\mathrm{C}$ & 9.24322 & -2.38520 & -2.46146 \\
\hline $\mathrm{C}$ & 9.21383 & -1.16607 & -3.23280 \\
\hline $\mathrm{C}$ & 7.95832 & -0.48411 & -3.37318 \\
\hline $\mathrm{C}$ & 7.93891 & 0.93352 & -3.29230 \\
\hline $\mathrm{C}$ & 9.17991 & 1.62399 & -3.08559 \\
\hline $\mathrm{C}$ & 9.18710 & 2.75102 & -2.18469 \\
\hline $\mathrm{C}$ & 7.95355 & 3.12043 & -1.54847 \\
\hline $\mathrm{C}$ & 7.98577 & 3.49008 & -0.17888 \\
\hline $\mathrm{C}$ & 9.24589 & 3.46311 & 0.51063 \\
\hline $\mathrm{C}$ & 9.27911 & 2.92672 & 1.84980 \\
\hline $\mathrm{C}$ & 8.04888 & 2.45546 & 2.41642 \\
\hline $\mathrm{C}$ & 8.06059 & 1.25440 & 3.17227 \\
\hline $\mathrm{C}$ & 9.30851 & 0.56915 & 3.34818 \\
\hline $\mathrm{C}$ & 9.31107 & -0.87183 & 3.27456 \\
\hline
\end{tabular}




\begin{tabular}{lrrr}
$\mathrm{C}$ & 10.53762 & -1.55183 & 3.04609 \\
$\mathrm{C}$ & 10.53395 & -2.68313 & 2.13475 \\
$\mathrm{C}$ & 10.50792 & -3.39633 & -0.60390 \\
$\mathrm{C}$ & 10.48382 & -2.85679 & -1.95253 \\
$\mathrm{C}$ & 10.42675 & -0.46757 & -3.47736 \\
$\mathrm{C}$ & 10.40758 & 0.98299 & -3.40751 \\
$\mathrm{C}$ & 10.42337 & 3.18803 & -1.63478 \\
$\mathrm{C}$ & 10.45542 & 3.55140 & -0.22910 \\
$\mathrm{C}$ & 10.51688 & 2.49964 & 2.40097 \\
$\mathrm{C}$ & 10.53120 & 1.27707 & 3.18527 \\
$\mathrm{C}$ & -11.72169 & 3.54444 & -0.26055 \\
$\mathrm{H}$ & -12.65476 & 3.77726 & 0.24071 \\
$\mathrm{C}$ & -11.62136 & 2.64028 & 2.56973 \\
$\mathrm{H}$ & -12.56564 & 3.14039 & 2.38425 \\
$\mathrm{C}$ & -11.59805 & 1.51319 & 3.35030 \\
$\mathrm{H}$ & -12.52437 & 1.14124 & 3.77407 \\
$\mathrm{C}$ & -11.63114 & -1.45571 & 3.25454 \\
$\mathrm{H}$ & -12.55909 & -1.07277 & 3.66438 \\
$\mathrm{C}$ & -11.66458 & -2.55738 & 2.43667 \\
$\mathrm{H}$ & -12.61866 & -3.02286 & 2.21469 \\
$\mathrm{C}$ & -11.74971 & 3.12350 & -1.56645 \\
$\mathrm{H}$ & -12.70420 & 3.03202 & -2.07282 \\
$\mathrm{C}$ & -11.75764 & 0.75374 & -3.36854 \\
$\mathrm{H}$ & -12.70100 & 1.28284 & -3.44815 \\
$\mathrm{C}$ & -11.75214 & -0.61738 & -3.40119 \\
$\mathrm{H}$ & -12.69090 & -1.15009 & -3.50535 \\
$\mathrm{C}$ & -11.73866 & -3.04491 & -1.69665 \\
$\mathrm{H}$ & -12.68600 & -2.95934 & -2.21734 \\
$\mathrm{C}$ & -11.72537 & -3.47927 & -0.39552 \\
$\mathrm{H}$ & -12.66188 & -3.72867 & 0.09129 \\
$\mathrm{C}$ & 11.70616 & 3.03831 & 1.80429 \\
$\mathrm{H}$ & 12.66266 & 2.91803 & 2.30092 \\
$\mathrm{C}$ & 11.67583 & 3.54949 & 0.52760 \\
$\mathrm{H}$ & 12.60934 & 3.81944 & 0.04614 \\
$\mathrm{C}$ & 11.61261 & 2.82129 & -2.34997 \\
$\mathrm{H}$ & 12.55662 & 3.29720 & -2.10824 \\
$\mathrm{C}$ & 11.60489 & 1.74981 & -3.21180 \\
$\mathrm{H}$ & 12.54272 & 1.40447 & -3.63283 \\
$\mathrm{C}$ & 11.73843 & 0.53925 & 3.42297 \\
$\mathrm{H}$ & 12.68230 & 1.06466 & 3.51895 \\
$\mathrm{C}$ & 11.74116 & -0.83462 & 3.35814 \\
$\mathrm{H}$ & 12.68735 & -1.36242 & 3.40424 \\
$\mathrm{H}$ & 11.73214 & -3.14105 & 1.49076 \\
$\mathrm{H}$ & 11.71999 & -3.08011 & 2.01046 \\
-3.48581 & 0.15900 \\
\hline & -3.68893 & -0.34154 \\
$\mathrm{H}$ & -0.80605 & -3.70954 \\
\hline
\end{tabular}


Structure of TS1 within the ONIOM scheme

\begin{tabular}{|c|c|c|c|}
\hline 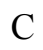 & -10.59406 & -3.10437 & 1498 \\
\hline $\mathrm{C}$ & -10.71700 & -2.80855 & -0.66509 \\
\hline $\mathrm{C}$ & -10.79448 & -0.67099 & -2.49738 \\
\hline C & -10.74658 & 0.74094 & -2.59279 \\
\hline $\mathrm{C}$ & -10.55580 & 3.13829 & -1.11539 \\
\hline C & -10.44435 & 3.69558 & 0.18229 \\
\hline $\mathrm{C}$ & -10.21082 & 3.09602 & 2.93152 \\
\hline $\mathrm{C}$ & -10.12558 & 2.02792 & 3.85769 \\
\hline $\mathrm{C}$ & -10.15978 & -0.78182 & 4.03705 \\
\hline $\mathrm{C}$ & -10.28058 & -1.97879 & 3.28944 \\
\hline $\mathrm{C}$ & -9.13745 & -2.50176 & 2.64666 \\
\hline $\mathrm{C}$ & -9.30661 & -3.12082 & 1.29461 \\
\hline $\mathrm{C}$ & -8.17468 & -3.22907 & 0.46460 \\
\hline $\mathrm{C}$ & -8.29525 & -2.93524 & -0.90486 \\
\hline $\mathrm{C}$ & -9.54696 & -2.53474 & -1.40950 \\
\hline $\mathrm{C}$ & -9.58346 & -1.38776 & -2.36948 \\
\hline $\mathrm{C}$ & -8.36522 & -0.75941 & -2.69624 \\
\hline $\mathrm{C}$ & -8.31459 & 0.64460 & -2.76308 \\
\hline $\mathrm{C}$ & -9.49054 & 1.38730 & -2.53749 \\
\hline $\mathrm{C}$ & -9.38995 & 2.65921 & -1.75752 \\
\hline $\mathrm{C}$ & -8.12264 & 3.06342 & -1.29359 \\
\hline $\mathrm{C}$ & -8.01565 & 3.65935 & -0.02404 \\
\hline $\mathrm{C}$ & -9.16728 & 3.78989 & 0.77698 \\
\hline $\mathrm{C}$ & -9.04614 & 3.48651 & 2.23815 \\
\hline $\mathrm{C}$ & -7.78697 & 3.09399 & 2.72786 \\
\hline $\mathrm{C}$ & -7.69895 & 1.99 & 3.6 \\
\hline $\mathrm{C}$ & -8.88673 & 1.36 & 4.01885 \\
\hline $\mathrm{C}$ & -8.90466 & -0.12978 & 4.09134 \\
\hline $\mathrm{C}$ & -7.73751 & -0.82999 & 3.72648 \\
\hline $\mathrm{C}$ & -7.85912 & -2.03545 & 3.01070 \\
\hline $\mathrm{C}$ & -6.66557 & -2.58456 & 2.32649 \\
\hline $\mathrm{C}$ & -6.82379 & -3.20660 & 1.07181 \\
\hline $\mathrm{C}$ & -5.69383 & -3.40453 & 0.25080 \\
\hline $\mathrm{C}$ & -5.81781 & -3.08675 & -1.20434 \\
\hline $\mathrm{C}$ & -7.06262 & -2.61177 & -1.66808 \\
\hline $\mathrm{C}$ & -7.09369 & -1.51233 & -2.54772 \\
\hline $\mathrm{C}$ & -5.88066 & -0.90808 & -2.93475 \\
\hline $\mathrm{C}$ & -5.82150 & 0.58578 & -2.96712 \\
\hline $\mathrm{C}$ & -6.99191 & 1.31407 & -2.66341 \\
\hline $\mathrm{C}$ & -6.89500 & 2.51560 & -1.93186 \\
\hline $\mathrm{C}$ & -5.62738 & 2.97195 & -1.50936 \\
\hline $\mathrm{C}$ & -5.52367 & 3.66798 & -0.18875 \\
\hline $\mathrm{C}$ & -6.67 & 3.75 & \\
\hline $\mathrm{C}$ & -6.57218 & 3.50 & 1.9 \\
\hline $\mathrm{C}$ & -5.31330 & 3.18999 & 2.53771 \\
\hline $\mathrm{C}$ & -5.20 & 1.94498 & 3.34897 \\
\hline $\mathrm{C}$ & & 1.27 & 3.70093 \\
\hline $\mathrm{C}$ & -6. & -0.13 & 3.73776 \\
\hline $\mathrm{C}$ & -5 . & -0.8 & 3.42521 \\
\hline $\mathrm{C}$ & -5.38 & -2.16 & 2.72740 \\
\hline $\mathrm{C}$ & -4 & -2.69 & 2.07279 \\
\hline $\mathrm{C}$ & -4.41313 & -3.33783 & 0.83092 \\
\hline $\mathrm{C}$ & -3.21049 & -3.50118 & -0.02610 \\
\hline $\mathrm{C}$ & -3.32342 & -3.19507 & -1.39554 \\
\hline $\mathrm{C}$ & -4.64358 & -2.73376 & -1.90443 \\
\hline
\end{tabular}




\begin{tabular}{|c|c|c|c|}
\hline $\mathrm{C}$ & -4.67449 & -1.62362 & -2.77158 \\
\hline$C$ & -3.38818 & -0.97520 & -3.12693 \\
\hline $\mathrm{C}$ & -3.33002 & 0.43233 & -3.12001 \\
\hline $\mathrm{C}$ & -4.56468 & 1.20521 & -2.81690 \\
\hline $\mathrm{C}$ & -4.46339 & 2.39641 & -2.06751 \\
\hline $\mathrm{C}$ & -3.12563 & 2.81641 & -1.57406 \\
\hline $\mathrm{C}$ & -3.03555 & 3.51468 & -0.35689 \\
\hline $\mathrm{C}$ & -4.26530 & 3.75105 & 0.43822 \\
\hline $\mathrm{C}$ & -4.17136 & 3.58832 & 1.84307 \\
\hline $\mathrm{C}$ & -2.83737 & 3.20351 & 2.38340 \\
\hline $\mathrm{C}$ & -2.69531 & 1.93349 & 2.87089 \\
\hline $\mathrm{C}$ & -3.96859 & 1.23228 & 3.30279 \\
\hline $\mathrm{C}$ & -4.00826 & -0.17135 & 3.35194 \\
\hline $\mathrm{C}$ & -2.79138 & -0.93987 & 3.03030 \\
\hline $\mathrm{C}$ & -2.92142 & -2.22096 & 2.45822 \\
\hline $\mathrm{C}$ & -1.79465 & -2.85579 & 1.91261 \\
\hline $\mathrm{C}$ & -1.93409 & -3.50824 & 0.57607 \\
\hline $\mathrm{C}$ & -0.78332 & -3.59469 & -0.23772 \\
\hline $\mathrm{C}$ & -0.89903 & -3.29924 & -1.61110 \\
\hline $\mathrm{C}$ & -2.15390 & -2.90033 & -2.12833 \\
\hline $\mathrm{C}$ & -2.18800 & -1.71786 & -3.04562 \\
\hline $\mathrm{C}$ & -0.96382 & -1.07610 & -3.33431 \\
\hline $\mathrm{C}$ & -0.90457 & 0.32979 & -3.28910 \\
\hline $\mathrm{C}$ & -2.07678 & 1.06166 & -3.01745 \\
\hline $\mathrm{C}$ & -1.96111 & 2.26230 & -2.14545 \\
\hline $\mathrm{C}$ & -0.69937 & 2.55537 & -1.58509 \\
\hline $\mathrm{C}$ & -0.63068 & 3.14500 & -0.26412 \\
\hline $\mathrm{C}$ & -1.75442 & 3.60567 & 0.32214 \\
\hline $\mathrm{C}$ & -1.72643 & 3.89763 & 1.75354 \\
\hline $\mathrm{C}$ & -0.09508 & 1.62944 & 683 \\
\hline $\mathrm{C}$ & -1.39183 & 1.18198 & 2.90145 \\
\hline $\mathrm{C}$ & -1.52823 & -0.3 & 910 \\
\hline $\mathrm{C}$ & -0.35 & -1.1 & 006 \\
\hline $\mathrm{C}$ & -0.5 & -2.4 & 415 \\
\hline $\mathrm{C}$ & 0.6 & -3.0 & 77 \\
\hline $\mathrm{C}$ & 0.5 & -3.5 & \\
\hline $\mathrm{C}$ & 1.7 & -3.7 & 843 \\
\hline $\mathrm{C}$ & 1.6 & 96 & -1.83418 \\
\hline $\mathrm{C}$ & 0. & 91 & -2.34885 \\
\hline $\mathrm{C}$ & 77 & -1.83234 & -3.19776 \\
\hline $\mathrm{C}$ & 1.58295 & -1.15801 & -3.52441 \\
\hline $\mathrm{C}$ & 1.60299 & 0.32731 & -3.42850 \\
\hline $\mathrm{C}$ & 0.41081 & 0.99263 & -3.08074 \\
\hline $\mathrm{C}$ & 0.47629 & 2.08161 & -2.18784 \\
\hline $\mathrm{C}$ & 1.73083 & 2.54168 & -1.73726 \\
\hline $\mathrm{C}$ & 1.84561 & 3.14187 & -0.37322 \\
\hline $\mathrm{C}$ & 0.62323 & 3.06053 & 0.48503 \\
\hline $\mathrm{C}$ & 0.73628 & 2.64450 & 1.79735 \\
\hline $\mathrm{C}$ & 2.12976 & 2.54349 & 2.32196 \\
\hline $\mathrm{C}$ & 2.22523 & 1.35528 & 3.12565 \\
\hline $\mathrm{C}$ & 0.99550 & 0.76331 & 3.14026 \\
\hline $\mathrm{C}$ & 0.87000 & -0.62212 & 3.15150 \\
\hline $\mathrm{C}$ & 2.09322 & -1.46066 & 3.04445 \\
\hline $\mathrm{C}$ & 1.99768 & -2.70943 & 2.25353 \\
\hline $\mathrm{C}$ & 3.14408 & -3.20377 & 1.62023 \\
\hline $\mathrm{C}$ & 3.00649 & -3.72637 & 0.23672 \\
\hline $\mathrm{C}$ & 4.22526 & -3.78423 & -0.60743 \\
\hline
\end{tabular}




\begin{tabular}{|c|c|c|c|}
\hline $\mathrm{C}$ & 4.12714 & -3.44122 & -1.96236 \\
\hline $\mathrm{C}$ & 2.81876 & -2.99706 & -2.49603 \\
\hline $\mathrm{C}$ & 2.79516 & -1.85232 & -3.32715 \\
\hline $\mathrm{C}$ & 4.07914 & -1.14582 & -3.60977 \\
\hline $\mathrm{C}$ & 4.09621 & 0.26604 & -3.56739 \\
\hline $\mathrm{C}$ & 2.83746 & 0.97509 & -3.22591 \\
\hline $\mathrm{C}$ & 2.89249 & 2.11183 & -2.39635 \\
\hline $\mathrm{C}$ & 4.20396 & 2.61960 & -1.95251 \\
\hline $\mathrm{C}$ & 4.32635 & 3.16860 & -0.66854 \\
\hline $\mathrm{C}$ & 3.12343 & 3.22429 & 0.21435 \\
\hline $\mathrm{C}$ & 3.26830 & 2.92621 & 1.58352 \\
\hline $\mathrm{C}$ & 4.60788 & 2.47119 & 2.06995 \\
\hline $\mathrm{C}$ & 4.68160 & 1.29000 & 2.84205 \\
\hline $\mathrm{C}$ & 3.41670 & 0.62904 & 3.23712 \\
\hline $\mathrm{C}$ & 3.34679 & -0.85092 & 3.21676 \\
\hline $\mathrm{C}$ & 4.57477 & -1.59036 & 2.86973 \\
\hline $\mathrm{C}$ & 4.47842 & -2.74373 & 2.07632 \\
\hline $\mathrm{C}$ & 5.69323 & -3.26942 & 1.41546 \\
\hline $\mathrm{C}$ & 5.56383 & -3.81251 & 0.03520 \\
\hline $\mathrm{C}$ & 6.71201 & -3.88239 & -0.77504 \\
\hline $\mathrm{C}$ & 6.60376 & -3.50037 & -2.20964 \\
\hline $\mathrm{C}$ & 5.35470 & -3.08491 & -2.70887 \\
\hline $\mathrm{C}$ & 5.29637 & -1.85522 & -3.54756 \\
\hline $\mathrm{C}$ & 6.49744 & -1.19412 & -3.85705 \\
\hline $\mathrm{C}$ & 6.51033 & 0.28638 & -3.84348 \\
\hline $\mathrm{C}$ & 5.32477 & 0.95811 & -3.50916 \\
\hline $\mathrm{C}$ & 5.41079 & 2.20986 & -2.70447 \\
\hline $\mathrm{C}$ & 6.67142 & 2.66850 & -2.28311 \\
\hline $\mathrm{C}$ & 6.81235 & 3.23036 & -0.91177 \\
\hline $\mathrm{C}$ & 5.67895 & 3.29150 & -0.07554 \\
\hline $\mathrm{C}$ & 5.82356 & 2.92150 & 1.35527 \\
\hline $\mathrm{C}$ & 7.07133 & 2.46699 & 1.80659 \\
\hline $\mathrm{C}$ & 7.11085 & 1.23876 & 2.64020 \\
\hline $\mathrm{C}$ & 5.90221 & 0.59097 & 2.96006 \\
\hline $\mathrm{C}$ & 5.88068 & -0.90421 & 3.01259 \\
\hline $\mathrm{C}$ & 7.05363 & -1.61281 & 2.69775 \\
\hline $\mathrm{C}$ & 6.95540 & -2.84735 & 1.87030 \\
\hline $\mathrm{C}$ & 8.16955 & -3.30894 & 1.15884 \\
\hline $\mathrm{C}$ & 8.05117 & -3.81896 & -0.13871 \\
\hline $\mathrm{C}$ & 9.26452 & -3.92097 & -0.98188 \\
\hline $\mathrm{C}$ & 9.15520 & -3.51100 & -2.40705 \\
\hline $\mathrm{C}$ & 7.83816 & -3.0 & -2.89067 \\
\hline $\mathrm{C}$ & 7.78424 & -1.90424 & -3.70130 \\
\hline $\mathrm{C}$ & 9.04217 & -1.21837 & -4.07179 \\
\hline $\mathrm{C}$ & 9.05 & 0.2 & -4.08708 \\
\hline $\mathrm{C}$ & 7.81008 & 0.98 & -3.71724 \\
\hline $\mathrm{C}$ & 7.88999 & 2.15354 & -2.95650 \\
\hline $\mathrm{C}$ & 9.22143 & 2.64711 & -2.53726 \\
\hline $\mathrm{C}$ & 9.36216 & 3.18848 & -1.15970 \\
\hline $\mathrm{C}$ & 8.16094 & 3.20702 & -0.29457 \\
\hline $\mathrm{C}$ & 8.28726 & 2.82102 & 1.04344 \\
\hline $\mathrm{C}$ & 9.60861 & 2.38388 & 1.54451 \\
\hline $\mathrm{C}$ & 9.65945 & 1.17742 & 2.41303 \\
\hline $\mathrm{C}$ & 8.38553 & 0.48693 & 2.71979 \\
\hline $\mathrm{C}$ & 8.35689 & -0.91100 & 2.74782 \\
\hline $\mathrm{C}$ & 9.60057 & -1.66376 & 2.47185 \\
\hline $\mathrm{C}$ & 9.50371 & -2.90329 & 1.65624 \\
\hline
\end{tabular}




$\begin{array}{lrrr}\mathrm{C} & 10.66184 & -3.37962 & 1.01295 \\ \mathrm{C} & 10.53678 & -3.91418 & -0.37674 \\ \mathrm{C} & 10.32577 & -3.11401 & -3.08300 \\ \mathrm{C} & 10.26621 & -1.90325 & -3.95759 \\ \mathrm{C} & 10.29028 & 0.93792 & -4.01152 \\ \mathrm{C} & 10.37695 & 2.19245 & -3.20376 \\ \mathrm{C} & 10.64236 & 3.19937 & -0.57146 \\ \mathrm{C} & 10.77327 & 2.76444 & 0.85231 \\ \mathrm{C} & 10.87743 & 0.48030 & 2.53141 \\ \mathrm{C} & 10.84578 & -1.01359 & 2.56857 \\ \mathrm{C} & -11.51640 & 3.53177 & 2.37553 \\ \mathrm{H} & -12.40058 & 3.56102 & 2.99790 \\ \mathrm{C} & -11.34416 & 1.33329 & 4.33774 \\ \mathrm{H} & -12.24472 & 1.88877 & 4.56295 \\ \mathrm{C} & -11.35890 & 0.00060 & 4.42824 \\ \mathrm{H} & -12.27156 & -0.49557 & 4.72894 \\ \mathrm{C} & -11.60757 & -2.48416 & 2.85225 \\ \mathrm{H} & -12.47782 & -2.37665 & 3.48502 \\ \mathrm{C} & -11.75705 & -3.00944 & 1.63140 \\ \mathrm{H} & -12.74320 & -3.31158 & 1.30550 \\ \mathrm{C} & -11.62666 & 3.81826 & 1.07331 \\ \mathrm{H} & -12.59801 & 4.07523 & 0.67342 \\ \mathrm{C} & -11.86680 & 2.69986 & -1.65396 \\ \mathrm{H} & -12.76758 & 3.25954 & -1.44158 \\ \mathrm{C} & -11.95668 & 1.56673 & -2.35631 \\ \mathrm{H} & -12.92847 & 1.23440 & -2.69541 \\ \mathrm{C} & -12.04916 & -1.37112 & -2.12216 \\ \mathrm{H} & -13.00631 & -1.03191 & -2.49426 \\ \mathrm{C} & -12.01286 & -2.38106 & -1.24689 \\ \mathrm{H} & -1.96205 & 5.55500 & 2.03529 \\ \mathrm{H} & -12.94027 & -2.83753 & -0.92818 \\ \mathrm{C} & 12.12251 & 1.19896 & 2.16463 \\ \mathrm{H} & 13.08610 & 0.82578 & 2.48311 \\ \mathrm{C} & 12.07334 & 2.27536 & 1.37170 \\ \mathrm{H} & 12.99762 & 2.74600 & 1.06521 \\ \mathrm{C} & 11.81546 & 3.15918 & -1.47674 \\ \mathrm{H} & 12.79317 & 3.45392 & -1.12028 \\ \mathrm{C} & 11.68992 & 2.68518 & -2.72080 \\ \mathrm{H} & 12.56915 & 2.60929 & -3.34571 \\ \mathrm{C} & 12.06221 & -1.80306 & 2.25781 \\ \mathrm{H} & 13.03697 & -1.45563 & 2.57154 \\ \mathrm{C} & 11.97521 & -2.91958 & 1.52636 \\ \mathrm{H} & 12.88171 & -3.44801 & 1.26452 \\ \mathrm{H} & 11.72250 & -3.96821 & -1.26632 \\ \mathrm{H} & 11.62359 & -4.25195 & -0.87514 \\ \mathrm{H} & -3.58643 & -2.54420 \\ \mathrm{H} & -3.56886 & -3.15993 \\ \mathrm{H} & -1.16930 & -4.31714 \\ \mathrm{H} & -1.70604 & -4.50504 \\ \mathrm{H} & 1.5372 & 1.37573\end{array}$


Structure of TS2 within the ONIOM scheme

\begin{tabular}{|c|c|c|c|}
\hline 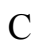 & 9685 & 3410 & 890 \\
\hline $\mathrm{C}$ & -10.52373 & -3.48683 & 0.38624 \\
\hline $\mathrm{C}$ & -10.54977 & -2.63410 & -2.30162 \\
\hline $\mathrm{C}$ & -10.55630 & -1.48593 & -3.12991 \\
\hline $\mathrm{C}$ & -10.59335 & 1.33015 & -3.08167 \\
\hline $\mathrm{C}$ & -10.61289 & 2.45905 & -2.22609 \\
\hline $\mathrm{C}$ & -10.57755 & 3.35009 & 0.45932 \\
\hline $\mathrm{C}$ & -10.52131 & 2.94414 & 1.81545 \\
\hline $\mathrm{C}$ & -10.43796 & 0.65602 & 3.44750 \\
\hline $\mathrm{C}$ & -10.44126 & -0.75925 & 3.40656 \\
\hline $\mathrm{C}$ & -9.22693 & -1.43776 & 3.16560 \\
\hline $\mathrm{C}$ & -9.25987 & -2.65480 & 2.29537 \\
\hline $\mathrm{C}$ & -8.06226 & -3.07123 & 1.68570 \\
\hline $\mathrm{C}$ & -8.08797 & -3.52517 & 0.35515 \\
\hline $\mathrm{C}$ & -9.31189 & -3.54318 & -0.33880 \\
\hline $\mathrm{C}$ & -9.32252 & -3.07673 & -1.76042 \\
\hline $\mathrm{C}$ & -8.11105 & -2.61931 & -2.31724 \\
\hline $\mathrm{C}$ & -8.11606 & -1.46068 & -3.11445 \\
\hline $\mathrm{C}$ & -9.33861 & -0.80579 & -3.36388 \\
\hline $\mathrm{C}$ & -9.35872 & 0.68915 & -3.34169 \\
\hline $\mathrm{C}$ & -8.15229 & 1.36995 & -3.08499 \\
\hline $\mathrm{C}$ & -8.17671 & 2.51734 & -2.27270 \\
\hline $\mathrm{C}$ & -9.39380 & 2.93 & -1.69789 \\
\hline $\mathrm{C}$ & -9.37822 & 3.41540 & -0.27980 \\
\hline $\mathrm{C}$ & -8.14 & 3.4 & 0.40117 \\
\hline $\mathrm{C}$ & -8.08 & 2.95 & 1.7 \\
\hline $\mathrm{C}$ & -9.27093 & 2.57356 & 2.36595 \\
\hline $\mathrm{C}$ & -9.22359 & 1.34 & 3.21658 \\
\hline $\mathrm{C}$ & -7.99789 & 0.65652 & 3.32228 \\
\hline $\mathrm{C}$ & -8.00438 & -0.75015 & 3.30568 \\
\hline $\mathrm{C}$ & -6.73494 & -1.46 & 3.03989 \\
\hline $\mathrm{C}$ & -6.76624 & -2.63820 & 2.25818 \\
\hline $\mathrm{C}$ & -5.56890 & -3.13104 & 1.70241 \\
\hline $\mathrm{C}$ & -5.59351 & -3.61 & 0.28841 \\
\hline $\mathrm{C}$ & -6.81578 & -3.547 & -0.40938 \\
\hline $\mathrm{C}$ & -6.82441 & -3.07732 & -1.73721 \\
\hline $\mathrm{C}$ & -5.61199 & -2.67055 & -2.33079 \\
\hline $\mathrm{C}$ & -5.61249 & -1.41386 & -3.14066 \\
\hline $\mathrm{C}$ & -6.83534 & -0.73 & -3.31781 \\
\hline $\mathrm{C}$ & -6.85141 & 0.676 & -3.30297 \\
\hline $\mathrm{C}$ & -5.642 & 1.38 & -3.10644 \\
\hline $\mathrm{C}$ & -5.68 & 2.63 & -2.30404 \\
\hline $\mathrm{C}$ & -6.90 & 3.01 & -1.70 \\
\hline $\mathrm{C}$ & -6.89 & 3.48 & -0.3 \\
\hline $\mathrm{C}$ & -5.67609 & 3.60 & \\
\hline $\mathrm{C}$ & & 3.03 & \\
\hline $\mathrm{C}$ & & 2.55 & \\
\hline $\mathrm{C}$ & & 1.37 & \\
\hline $\mathrm{C}$ & & 5 & \\
\hline $\mathrm{C}$ & & -0.79 & \\
\hline $\mathrm{C}$ & & -1.4 & 429 \\
\hline$C$ & -4.342 & -2.67 & 2.22231 \\
\hline$C$ & 207 & -3.16308 & 1.62393 \\
\hline ( & & -3.61 & 0.29166 \\
\hline 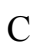 & -4.38640 & -3.58745 & -0.44053 \\
\hline
\end{tabular}




\begin{tabular}{|c|c|c|c|}
\hline $\mathrm{C}$ & -4.39467 & -3.09656 & -1.76120 \\
\hline $\mathrm{C}$ & -3.11381 & -2.62583 & -2.34358 \\
\hline $\mathrm{C}$ & -3.11225 & -1.41685 & -3.06615 \\
\hline $\mathrm{C}$ & -4.39830 & -0.70511 & -3.27068 \\
\hline $\mathrm{C}$ & -4.40730 & 0.70222 & -3.23151 \\
\hline $\mathrm{C}$ & -3.12634 & 1.42001 & -2.95202 \\
\hline $\mathrm{C}$ & -3.17536 & 2.62906 & -2.24403 \\
\hline $\mathrm{C}$ & -4.48593 & 3.11000 & -1.73929 \\
\hline $\mathrm{C}$ & -4.50698 & 3.65584 & -0.44397 \\
\hline $\mathrm{C}$ & -3.22554 & 3.75028 & 0.26752 \\
\hline $\mathrm{C}$ & -3.03042 & 3.02037 & 1.43895 \\
\hline $\mathrm{C}$ & -4.30391 & 2.53931 & 2.10736 \\
\hline $\mathrm{C}$ & -4.27923 & 1.34936 & 2.85291 \\
\hline $\mathrm{C}$ & -3.00076 & 0.62074 & 2.98986 \\
\hline $\mathrm{C}$ & -3.02877 & -0.78161 & 3.12327 \\
\hline $\mathrm{C}$ & -1.83721 & -1.51252 & 3.01167 \\
\hline $\mathrm{C}$ & -1.85060 & -2.76576 & 2.19783 \\
\hline $\mathrm{C}$ & -0.64221 & -3.17702 & 1.59805 \\
\hline $\mathrm{C}$ & -0.66486 & -3.64227 & 0.26808 \\
\hline $\mathrm{C}$ & -1.88828 & -3.65935 & -0.43565 \\
\hline $\mathrm{C}$ & -1.89761 & -3.12476 & -1.83372 \\
\hline $\mathrm{C}$ & -0.68395 & -2.63506 & -2.36004 \\
\hline $\mathrm{C}$ & -0.68252 & -1.41007 & -3.05747 \\
\hline $\mathrm{C}$ & -1.89666 & -0.72775 & -3.25183 \\
\hline $\mathrm{C}$ & -1.89204 & 0.76235 & -3.14656 \\
\hline $\mathrm{C}$ & -0.66552 & 1.42307 & -2.87100 \\
\hline $\mathrm{C}$ & -0.66 & 2.66 & -2.02824 \\
\hline $\mathrm{C}$ & -1.92777 & 3.20 & 009 \\
\hline $\mathrm{C}$ & -2.08726 & 4.0 & -0.55411 \\
\hline $\mathrm{C}$ & -0.36340 & $2.8^{\prime}$ & 1.4 \\
\hline $\mathrm{C}$ & -1.72449 & 2.5 & 1.8 \\
\hline $\mathrm{C}$ & -1.77266 & 47 & 2.7 \\
\hline $\mathrm{C}$ & -0.58519 & 0.5 & 3.0 \\
\hline $\mathrm{C}$ & -0.61969 & -0.8 & 3. \\
\hline $\mathrm{C}$ & 0.6 & -1. & 3. \\
\hline $\mathrm{C}$ & 0.6 & -2 & 2.1 \\
\hline $\mathrm{C}$ & 1.8 & -3.1 & 1. \\
\hline $\mathrm{C}$ & 1.8 & -3 & 0 \\
\hline $\mathrm{C}$ & 0.60149 & -3.62998 & -0 . \\
\hline $\mathrm{C}$ & 0.59376 & -3.12262 & -1. \\
\hline $\mathrm{C}$ & 1.86570 & -2.63938 & 507 \\
\hline $\mathrm{C}$ & 1.82666 & -1.33465 & 9191 \\
\hline $\mathrm{C}$ & 0.58954 & -0.65822 & -3.20521 \\
\hline $\mathrm{C}$ & 0.55954 & 0.74807 & -3.06725 \\
\hline $\mathrm{C}$ & 1.76853 & 1.43176 & -2.84994 \\
\hline $\mathrm{C}$ & 1.76749 & 2.61342 & -1.94822 \\
\hline $\mathrm{C}$ & 0.47241 & 2.99233 & -1.30966 \\
\hline $\mathrm{C}$ & 0.48482 & 3.49737 & 0.13222 \\
\hline $\mathrm{C}$ & 1.87886 & 3.51991 & 0.67720 \\
\hline $\mathrm{C}$ & 1.92876 & 2.90204 & 1.97472 \\
\hline $\mathrm{C}$ & 0.74413 & 2.41224 & 2.28916 \\
\hline $\mathrm{C}$ & 0.64430 & 1.22966 & 3.03159 \\
\hline $\mathrm{C}$ & 1.89883 & 0.52332 & 3.43756 \\
\hline $\mathrm{C}$ & 1.90464 & -0.95654 & 3.40146 \\
\hline $\mathrm{C}$ & 3.11124 & -1.63367 & 3.17209 \\
\hline $\mathrm{C}$ & 3.09584 & -2.79487 & 2.24594 \\
\hline $\mathrm{C}$ & 4.37349 & -3.19578 & 1.60760 \\
\hline
\end{tabular}




\begin{tabular}{|c|c|c|c|}
\hline $\mathrm{C}$ & 4.36370 & -3.60130 & 0.26609 \\
\hline $\mathrm{C}$ & 3.08584 & -3.58456 & -0.47946 \\
\hline $\mathrm{C}$ & 3.08310 & -3.04699 & -1.78875 \\
\hline $\mathrm{C}$ & 4.36032 & -2.52069 & -2.34974 \\
\hline $\mathrm{C}$ & 4.32950 & -1.30906 & -3.07497 \\
\hline $\mathrm{C}$ & 3.02573 & -0.60829 & -3.22461 \\
\hline $\mathrm{C}$ & 2.98769 & 0.79369 & -3.12755 \\
\hline $\mathrm{C}$ & 4.24879 & 1.53522 & -2.93966 \\
\hline $\mathrm{C}$ & 4.25895 & 2.65712 & -2.10154 \\
\hline $\mathrm{C}$ & 2.99238 & 3.06817 & -1.42670 \\
\hline $\mathrm{C}$ & 3.04846 & 3.52358 & -0.09904 \\
\hline $\mathrm{C}$ & 4.35782 & 3.46930 & 0.61539 \\
\hline $\mathrm{C}$ & 4.40769 & 2.88234 & 1.89782 \\
\hline $\mathrm{C}$ & 3.13551 & 2.44330 & 2.51929 \\
\hline $\mathrm{C}$ & 3.11918 & 1.20376 & 3.31821 \\
\hline $\mathrm{C}$ & 4.39419 & 0.47585 & 3.47318 \\
\hline $\mathrm{C}$ & 4.39388 & -0.92376 & 3.39180 \\
\hline $\mathrm{C}$ & 5.67098 & -1.63392 & 3.15045 \\
\hline $\mathrm{C}$ & 5.66074 & -2.80846 & 2.23546 \\
\hline $\mathrm{C}$ & 6.86599 & -3.20726 & 1.62858 \\
\hline $\mathrm{C}$ & 6.84988 & -3.62234 & 0.19895 \\
\hline $\mathrm{C}$ & 5.62852 & -3.60668 & -0.49972 \\
\hline $\mathrm{C}$ & 5.59171 & -3.00962 & -1.86410 \\
\hline $\mathrm{C}$ & 6.79129 & -2.54031 & -2.42502 \\
\hline $\mathrm{C}$ & 6.75806 & -1.28353 & -3.20667 \\
\hline $\mathrm{C}$ & 5.53057 & -0.61482 & -3.33840 \\
\hline $\mathrm{C}$ & 5.52027 & 0.87498 & -3.30939 \\
\hline $\mathrm{C}$ & 6.73215 & 1.56380 & -3.12014 \\
\hline $\mathrm{C}$ & 6.75308 & 2.75013 & -2.22137 \\
\hline $\mathrm{C}$ & 5.55915 & 3.15142 & -1.58991 \\
\hline $\mathrm{C}$ & 5.60760 & 3.57067 & -0.16732 \\
\hline $\mathrm{C}$ & 6.83066 & 3.50496 & 0.51373 \\
\hline $\mathrm{C}$ & 6.84663 & 2.90957 & 1.87400 \\
\hline $\mathrm{C}$ & 5.63881 & 2.44776 & 2.43371 \\
\hline $\mathrm{C}$ & 5.66374 & 1.21832 & 3.28448 \\
\hline $\mathrm{C}$ & 6.87986 & 0.53024 & 3.44430 \\
\hline $\mathrm{C}$ & 6.88276 & -0.95744 & 3.37867 \\
\hline $\mathrm{C}$ & 8.15989 & -1.63798 & 3.06075 \\
\hline $\mathrm{C}$ & 8.15241 & -2.74306 & 2.20264 \\
\hline $\mathrm{C}$ & 9.42608 & -3.18 & 1.58785 \\
\hline $\mathrm{C}$ & 9.40734 & -3.5 & 0.15302 \\
\hline $\mathrm{C}$ & 8.11507 & -3.50848 & -0.56428 \\
\hline $\mathrm{C}$ & 8.08489 & -2.97618 & -1.85640 \\
\hline $\mathrm{C}$ & 9.34516 & -2.51249 & -2.47791 \\
\hline $\mathrm{C}$ & 9.31302 & -1.26170 & -3.28255 \\
\hline $\mathrm{C}$ & 8.02394 & -0.54317 & -3.40566 \\
\hline $\mathrm{C}$ & 8.01238 & 0.85497 & -3.36718 \\
\hline $\mathrm{C}$ & 9.29348 & 1.57783 & -3.19660 \\
\hline $\mathrm{C}$ & 9.31420 & 2.75300 & -2.28595 \\
\hline $\mathrm{C}$ & 8.05142 & 3.13164 & -1.61307 \\
\hline $\mathrm{C}$ & 8.08844 & 3.49439 & -0.26283 \\
\hline $\mathrm{C}$ & 9.38146 & 3.47366 & 0.45466 \\
\hline $\mathrm{C}$ & 9.40618 & 2.91396 & 1.83257 \\
\hline $\mathrm{C}$ & 8.13623 & 2.40613 & 2.40205 \\
\hline $\mathrm{C}$ & 8.15216 & 1.23861 & 3.17151 \\
\hline $\mathrm{C}$ & 9.43698 & 0.53759 & 3.38968 \\
\hline $\mathrm{C}$ & 9.44059 & -0.94838 & 3.33626 \\
\hline
\end{tabular}




\begin{tabular}{|c|c|c|c|}
\hline & 10.65715 & 1048 & 305 \\
\hline$C$ & 10.65026 & -2.78588 & 16053 \\
\hline & 10.61113 & -3.51374 & -0.57606 \\
\hline & 10.57807 & -2.95319 & -1.96142 \\
\hline & 10.51948 & -0.57389 & -3.51205 \\
\hline & 10.50857 & 0.92003 & -3.47347 \\
\hline & 10.54565 & 3.14616 & -1.72538 \\
\hline C & 10.58218 & 3.51680 & -0.27793 \\
\hline & 10.63428 & 2.47013 & 2.35976 \\
\hline & 10.64957 & 1.22329 & 3.18373 \\
\hline C & & 3.33027 & -0.30212 \\
\hline 11 & & & \\
\hline C & -11.74173 & & \\
\hline$\Pi$ & & & \\
\hline C & -11 & & \\
\hline H & & & \\
\hline 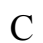 & & & \\
\hline$\Pi$ & & & \\
\hline$C$ & & & \\
\hline 1 & & & \\
\hline$C$ & & & \\
\hline $\mathrm{H}$ & & & \\
\hline$C$ & & & \\
\hline $\mathrm{H}$ & & & \\
\hline & & & \\
\hline $\mathrm{F}$ & & & \\
\hline & & & 699 \\
\hline & & & 266 \\
\hline & & & 995 \\
\hline & & & 933 \\
\hline$c$ & & & 1.7 \\
\hline & 12 & & 763 \\
\hline $\mathrm{C}$ & 11. & 28 & 150 \\
\hline $\mathrm{H}$ & 12. & & 637 \\
\hline $\mathrm{C}$ & 11. & 52 & 159 \\
\hline 1 & 12. & 76 & -2 \\
\hline 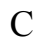 & 11. & & -3 \\
\hline $\mathrm{H}$ & 12. & & -3.70135 \\
\hline $\mathrm{C}$ & 11. & 02 & 3.38839 \\
\hline $\mathrm{H}$ & 12. & 0.99356 & 945 \\
\hline$c$ & 11.91384 & 0.86672 & 261 \\
\hline . & 12.85353 & 74 & 3.43632 \\
\hline 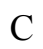 & 11.89862 & -3.21194 & 1.48202 \\
\hline $\mathrm{H}$ & 12.84390 & & 2.00686 \\
\hline 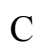 & 11.88069 & & 0.18868 \\
\hline- & 12.81139 & -3.79700 & -0.30469 \\
\hline $\mathrm{C}$ & 11.81544 & -2.44253 & -2.59962 \\
\hline- & 12.76228 & -2.93779 & -2.43313 \\
\hline & 11.78735 & -1.32367 & -3.33271 \\
\hline & 12.71271 & & -3.74178 \\
\hline & & & \\
\hline & -0.11396 & 4.66437 & 0.6930 \\
\hline
\end{tabular}


Structure of TS3 within the ONIOM scheme

\begin{tabular}{|c|c|c|c|}
\hline 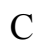 & 1229 & -3.16166 & 9093 \\
\hline $\mathrm{C}$ & 10.52299 & -3.49903 & -0.11452 \\
\hline $\mathrm{C}$ & 10.53593 & -2.46374 & 2.51267 \\
\hline $\mathrm{C}$ & 10.54436 & -1.27155 & 3.27652 \\
\hline $\mathrm{C}$ & 10.58727 & 1.53395 & 3.05395 \\
\hline $\mathrm{C}$ & 10.60908 & 2.59462 & 2.11535 \\
\hline $\mathrm{C}$ & 10.59789 & 3.26480 & -0.63297 \\
\hline $\mathrm{C}$ & 10.56520 & 2.76030 & -1.95656 \\
\hline $\mathrm{C}$ & 10.50473 & 0.38245 & -3.46190 \\
\hline $\mathrm{C}$ & 10.49666 & -1.02799 & -3.33959 \\
\hline $\mathrm{C}$ & 9.27348 & -1.68183 & -3.07265 \\
\hline $\mathrm{C}$ & 9.28423 & -2.81912 & -2.10048 \\
\hline $\mathrm{C}$ & 8.07599 & -3.16535 & -1.46805 \\
\hline $\mathrm{C}$ & 8.08669 & -3.50488 & -0.10336 \\
\hline $\mathrm{C}$ & 9.30470 & -3.48585 & 0.60102 \\
\hline $\mathrm{C}$ & 9.30942 & -2.92944 & 1.98999 \\
\hline $\mathrm{C}$ & 8.09756 & -2.43528 & 2.51324 \\
\hline $\mathrm{C}$ & 8.10421 & -1.24019 & 3.25444 \\
\hline $\mathrm{C}$ & 9.32818 & -0.57612 & 3.47048 \\
\hline $\mathrm{C}$ & 9.35186 & 0.91476 & 3.35716 \\
\hline $\mathrm{C}$ & 8.14763 & 1.57773 & 3.05008 \\
\hline $\mathrm{C}$ & 8.17320 & 2.64858 & 2.13925 \\
\hline $\mathrm{C}$ & 9.39252 & 3.01867 & 1.53878 \\
\hline $\mathrm{C}$ & 9.38720 & 3.37358 & 0.08462 \\
\hline $\mathrm{C}$ & 8.16331 & 3.32312 & -0.60882 \\
\hline $\mathrm{C}$ & 8.12698 & 2.80252 & -1.91477 \\
\hline $\mathrm{C}$ & 9.32489 & 2.35605 & -2.50393 \\
\hline $\mathrm{C}$ & 9.29081 & 1.08897 & -3.29723 \\
\hline $\mathrm{C}$ & 8.06440 & 0.40102 & -3.39843 \\
\hline $\mathrm{C}$ & 8.05808 & -1.00076 & -3.28842 \\
\hline $\mathrm{C}$ & 6.77654 & -1.68295 & -2.98524 \\
\hline $\mathrm{C}$ & 6.78821 & -2.77162 & -2.09057 \\
\hline $\mathrm{C}$ & 5.58070 & -3.19367 & -1.50135 \\
\hline $\mathrm{C}$ & 5.59251 & -3.56107 & -0.05219 \\
\hline $\mathrm{C}$ & 6.80928 & -3.45769 & 0.64908 \\
\hline $\mathrm{C}$ & 6.81230 & -2.91310 & 1.94858 \\
\hline $\mathrm{C}$ & 5.59880 & -2.47034 & 2.51390 \\
\hline $\mathrm{C}$ & 5.59988 & -1.18223 & 3.27263 \\
\hline $\mathrm{C}$ & 6.82517 & -0.49768 & 3.41958 \\
\hline $\mathrm{C}$ & 6.84642 & 0.907 & 3.32378 \\
\hline $\mathrm{C}$ & 5.64 & 1.60 & 3.08451 \\
\hline $\mathrm{C}$ & 5.68 & 2.77 & 2.15 \\
\hline $\mathrm{C}$ & 6.90 & 3.08 & \\
\hline $\mathrm{C}$ & 6.89739 & 3.41 & \\
\hline $\mathrm{C}$ & 5.67863 & 3.44 & -0.5 \\
\hline $\mathrm{C}$ & 5.62234 & 2.85 & -1.9 \\
\hline $\mathrm{C}$ & 6.820 & 2.34 & -2.4 \\
\hline $\mathrm{C}$ & $6.78^{\prime}$ & 1.13 & \\
\hline $\mathrm{C}$ & $5.55^{\circ}$ & 0.46 & \\
\hline $\mathrm{C}$ & 5.55 & -1.0 & -3.25919 \\
\hline $\mathrm{C}$ & 4.348 & -1.6 & -2.94542 \\
\hline $\mathrm{C}$ & 4.36112 & -2.77 & -2.07007 \\
\hline $\mathrm{C}$ & 308668 & -3.19192 & -1.43702 \\
\hline $\mathrm{C}$ & & -3.54640 & -0.07392 \\
\hline$C_{1}$ & 4.38262 & -3.47486 & 0.66343 \\
\hline
\end{tabular}




\begin{tabular}{|c|c|c|c|}
\hline $\mathrm{C}$ & 4.38424 & -2.91428 & 1.95641 \\
\hline $\mathrm{C}$ & 3.10247 & -2.41250 & 2.50877 \\
\hline $\mathrm{C}$ & 3.09772 & -1.17598 & 3.18396 \\
\hline $\mathrm{C}$ & 4.38664 & -0.46351 & 3.37123 \\
\hline $\mathrm{C}$ & 4.40194 & 0.94047 & 3.27098 \\
\hline $\mathrm{C}$ & 3.12311 & 1.65185 & 2.96360 \\
\hline $\mathrm{C}$ & 3.18296 & 2.80865 & 2.17952 \\
\hline $\mathrm{C}$ & 4.48334 & 3.17860 & 1.55407 \\
\hline $\mathrm{C}$ & 4.48884 & 3.51316 & 0.17759 \\
\hline $\mathrm{C}$ & 3.19838 & 3.50654 & -0.55662 \\
\hline $\mathrm{C}$ & 3.09769 & 2.88979 & -1.80526 \\
\hline $\mathrm{C}$ & 4.37474 & 2.36725 & -2.39256 \\
\hline $\mathrm{C}$ & 4.34640 & 1.15373 & -3.10400 \\
\hline $\mathrm{C}$ & 3.05363 & 0.43240 & -3.23405 \\
\hline $\mathrm{C}$ & 3.06350 & -0.97561 & -3.18536 \\
\hline $\mathrm{C}$ & 1.85861 & -1.67173 & -2.98363 \\
\hline $\mathrm{C}$ & 1.86689 & -2.84002 & -2.04826 \\
\hline $\mathrm{C}$ & 0.65703 & -3.20261 & -1.42047 \\
\hline $\mathrm{C}$ & 0.67420 & -3.57447 & -0.06111 \\
\hline $\mathrm{C}$ & 1.89089 & -3.54282 & 0.64780 \\
\hline $\mathrm{C}$ & 1.89097 & -2.93215 & 2.01442 \\
\hline $\mathrm{C}$ & 0.67554 & -2.41560 & 2.50609 \\
\hline $\mathrm{C}$ & 0.66711 & -1.15828 & 3.14259 \\
\hline $\mathrm{C}$ & 1.88042 & -0.47475 & 3.32780 \\
\hline $\mathrm{C}$ & 1.87886 & 1.00859 & 3.16558 \\
\hline $\mathrm{C}$ & 0.65436 & 1.6 & 2.82817 \\
\hline $\mathrm{C}$ & 0.68677 & $2.8^{\circ}$ & 1.94254 \\
\hline $\mathrm{C}$ & 1.93845 & 3.30 & 1.53273 \\
\hline $\mathrm{C}$ & 2.09682 & 3.78 & 0.23506 \\
\hline $\mathrm{C}$ & 0.57676 & 2.9 & -1.99536 \\
\hline $\mathrm{C}$ & 1.82592 & 2.4 & -2.38840 \\
\hline $\mathrm{C}$ & 1.82994 & 1.1 & -3.08304 \\
\hline $\mathrm{C}$ & 0.61385 & 0.4 & -3.25150 \\
\hline $\mathrm{C}$ & 0.63922 & -1. & -3.22036 \\
\hline $\mathrm{C}$ & -0.62285 & -1. & -2.98674 \\
\hline $\mathrm{C}$ & -0.63253 & -2.8 & -2.04772 \\
\hline $\mathrm{C}$ & -1.85573 & -3 & -1.48393 \\
\hline $\mathrm{C}$ & -1.86767 & -3 . & -0.04373 \\
\hline $\mathrm{C}$ & -0.5 & -3 . & 0.69359 \\
\hline $\mathrm{C}$ & -0.59443 & -2.9 & 1.96181 \\
\hline $\mathrm{C}$ & -1.86834 & -2.42577 & 2.50770 \\
\hline $\mathrm{C}$ & -1.83931 & -1.08434 & 3.15442 \\
\hline $\mathrm{C}$ & -0.60692 & -0.39769 & 3.23919 \\
\hline $\mathrm{C}$ & -0.57981 & 1.00370 & 3.04233 \\
\hline $\mathrm{C}$ & -1.79876 & 1.68242 & 2.82080 \\
\hline $\mathrm{C}$ & -1.82346 & 2.86566 & 1.91219 \\
\hline $\mathrm{C}$ & -0.54360 & 3.24307 & 1.22542 \\
\hline $\mathrm{C}$ & -0.66515 & 3.67105 & -0.09125 \\
\hline $\mathrm{C}$ & -1.95987 & 3.53860 & -0.77568 \\
\hline $\mathrm{C}$ & -1.93544 & 2.81863 & -2.06974 \\
\hline $\mathrm{C}$ & -0.68501 & 2.31943 & -2.50372 \\
\hline $\mathrm{C}$ & -0.62372 & 1.07814 & -3.15660 \\
\hline $\mathrm{C}$ & -1.89613 & 0.35392 & -3.44619 \\
\hline $\mathrm{C}$ & -1.89521 & -1.12210 & -3.35827 \\
\hline $\mathrm{C}$ & -3.09852 & -1.79305 & -3.09328 \\
\hline $\mathrm{C}$ & -3.07836 & -2.89654 & -2.10103 \\
\hline $\mathrm{C}$ & -4.35504 & -3.26476 & -1.43978 \\
\hline
\end{tabular}




\begin{tabular}{|c|c|c|c|}
\hline$C$ & -4.34671 & -3.57536 & -0.07338 \\
\hline $\mathrm{C}$ & -3.07313 & -3.49684 & 0.67145 \\
\hline $\mathrm{C}$ & -3.07871 & -2.87604 & 1.94352 \\
\hline $\mathrm{C}$ & -4.35946 & -2.33212 & 2.47308 \\
\hline $\mathrm{C}$ & -4.34100 & -1.08756 & 3.14201 \\
\hline $\mathrm{C}$ & -3.04316 & -0.36436 & 3.25963 \\
\hline $\mathrm{C}$ & -3.01715 & 1.03565 & 3.12204 \\
\hline $\mathrm{C}$ & -4.29514 & 1.75167 & 2.91849 \\
\hline $\mathrm{C}$ & -4.32694 & 2.83327 & 2.03288 \\
\hline $\mathrm{C}$ & -3.06976 & 3.23393 & 1.34613 \\
\hline $\mathrm{C}$ & -3.13357 & 3.55050 & -0.02589 \\
\hline $\mathrm{C}$ & -4.41918 & 3.41476 & -0.74569 \\
\hline $\mathrm{C}$ & -4.43668 & 2.75648 & -1.99033 \\
\hline $\mathrm{C}$ & -3.14337 & 2.30054 & -2.57203 \\
\hline $\mathrm{C}$ & -3.11756 & 1.03408 & -3.32925 \\
\hline $\mathrm{C}$ & -4.39034 & 0.29484 & -3.46886 \\
\hline $\mathrm{C}$ & -4.38377 & -1.10061 & -3.34346 \\
\hline $\mathrm{C}$ & -5.65714 & -1.80816 & -3.07456 \\
\hline $\mathrm{C}$ & -5.64271 & -2.92777 & -2.09343 \\
\hline $\mathrm{C}$ & -6.84649 & -3.29442 & -1.46338 \\
\hline $\mathrm{C}$ & -6.83096 & -3.61253 & -0.00912 \\
\hline $\mathrm{C}$ & -5.61231 & -3.54071 & 0.68891 \\
\hline $\mathrm{C}$ & -5.58375 & -2.86197 & 2.01492 \\
\hline $\mathrm{C}$ & -6.78853 & -2.38180 & 2.55208 \\
\hline $\mathrm{C}$ & -6.77070 & -1.09365 & 3.28163 \\
\hline $\mathrm{C}$ & -5.55112 & -0.40243 & 3.38504 \\
\hline $\mathrm{C}$ & -5.56097 & 1.08482 & 3.30753 \\
\hline $\mathrm{C}$ & -6.78494 & 1.74484 & 3.08163 \\
\hline $\mathrm{C}$ & -6.82217 & 2.87512 & 2.11462 \\
\hline $\mathrm{C}$ & -5.63177 & 3.25707 & 1.46982 \\
\hline $\mathrm{C}$ & -5.67402 & 3.55714 & 0.01601 \\
\hline $\mathrm{C}$ & -6.88680 & 3.43418 & -0.67001 \\
\hline $\mathrm{C}$ & -6.88015 & 2.77129 & -1.99862 \\
\hline $\mathrm{C}$ & -5.66005 & 2.29300 & -2.52245 \\
\hline $\mathrm{C}$ & -5.66762 & 1.03340 & -3.32389 \\
\hline $\mathrm{C}$ & -6.87849 & 0.33096 & -3.46995 \\
\hline $\mathrm{C}$ & -6.87217 & -1.15232 & -3.34037 \\
\hline $\mathrm{C}$ & -8.14562 & -1.82513 & -2.99118 \\
\hline $\mathrm{C}$ & -8.13389 & -2.87593 & -2.06759 \\
\hline $\mathrm{C}$ & -9.40599 & -3.28411 & -1.42804 \\
\hline $\mathrm{C}$ & -9.38810 & -3.5 & 0.02869 \\
\hline $\mathrm{C}$ & -8.09 & -3.4 & 343 \\
\hline $\mathrm{C}$ & -8.07 & -2.8 & 528 \\
\hline $\mathrm{C}$ & -9.3 & -2.3 & 2.6 \\
\hline $\mathrm{C}$ & -9 . & -1 . & 3.3 \\
\hline $\mathrm{C}$ & -8.04 & -0.36 & 3.45362 \\
\hline $\mathrm{C}$ & -8.05647 & 1.02936 & 3.35651 \\
\hline $\mathrm{C}$ & -9.34812 & 1.7 & 3.13919 \\
\hline $\mathrm{C}$ & -9.38167 & 2.83554 & 2.15392 \\
\hline $\mathrm{C}$ & -8.12019 & 3.18917 & 1.46786 \\
\hline $\mathrm{C}$ & -8.15018 & 3.45693 & 0.09507 \\
\hline $\mathrm{C}$ & -9.43577 & 3.37959 & -0.62984 \\
\hline $\mathrm{C}$ & -9.44143 & 2.75721 & -1.98087 \\
\hline $\mathrm{C}$ & -8.16094 & 2.23701 & -2.51745 \\
\hline $\mathrm{C}$ & -8.15981 & 1.03986 & -3.23959 \\
\hline $\mathrm{C}$ & -9.43747 & 0.31894 & -3.43796 \\
\hline $\mathrm{C}$ & -9.42989 & -1.16240 & -3.30997 \\
\hline
\end{tabular}




\begin{tabular}{|c|c|c|c|}
\hline & -10.64226 & -1.81934 & -3.02422 \\
\hline 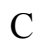 & -10.63098 & -2.93242 & -2.02734 \\
\hline$C$ & -10.59428 & -3.49393 & 0.75053 \\
\hline$C$ & -10.56908 & -2.86875 & 2.10836 \\
\hline C & -10.54352 & -0.42730 & 3.55853 \\
\hline a & -10.55485 & 1.06244 & 3.44549 \\
\hline $\mathrm{C}$ & -10.61408 & 3.17003 & 1.55831 \\
\hline $\mathrm{C}$ & -10.64305 & 3.44876 & 0.08973 \\
\hline a & -10.66085 & 2.28113 & -2.49957 \\
\hline 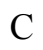 & -10.65774 & 1.00255 & -3.27295 \\
\hline C & 11.86299 & 3.31929 & 0.14234 \\
\hline & 12.79987 & 3.55560 & -0.34352 \\
\hline 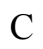 & 11.79820 & 2.27772 & -2.62501 \\
\hline 1 & 12.73901 & & \\
\hline 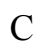 & 11.76839 & & \\
\hline 1 & 12. & & \\
\hline 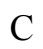 & & & \\
\hline 1 & & & \\
\hline 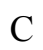 & & & \\
\hline 1 & 12 & & \\
\hline$C$ & & & \\
\hline 1 & & & \\
\hline$c$ & 11. & & \\
\hline 1 & & & \\
\hline $\mathrm{C}$ & 11.8 & & \\
\hline $\mathrm{H}$ & 12. & & \\
\hline $\mathrm{C}$ & 11. & & \\
\hline $\mathrm{H}$ & & & \\
\hline $\mathrm{C}$ & & & \\
\hline $\mathrm{H}$ & & & \\
\hline $\mathrm{C}$ & & & 586 \\
\hline $\mathrm{H}$ & & & \\
\hline $\mathrm{C}$ & & & \\
\hline$\Pi$ & & & \\
\hline$c$ & & & \\
\hline$\Pi$ & & & \\
\hline$c$ & & & \\
\hline $\mathrm{H}$ & & & \\
\hline $\mathrm{C}$ & & & \\
\hline $\mathrm{H}$ & & & \\
\hline $\mathrm{C}$ & & & \\
\hline 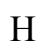 & & & \\
\hline $\mathrm{C}$ & & & \\
\hline 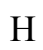 & & & 212 \\
\hline $\mathrm{C}$ & -11 & & 80 \\
\hline 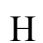 & -12 & & \\
\hline $\mathrm{C}$ & -11.81 & & 19 \\
\hline $\mathrm{H}$ & -12.7 & & 2.58219 \\
\hline $\mathrm{C}$ & -11.80045 & & 3.41334 \\
\hline $\mathrm{H}$ & -12.73056 & -0.8 & 3.80829 \\
\hline $\mathrm{O}$ & & & 0.87383 \\
\hline 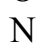 & 0.37460 & 3.94681 & -1.02059 \\
\hline
\end{tabular}


Structure of TS4 within the ONIOM scheme

\begin{tabular}{|c|c|c|c|}
\hline $\mathrm{r}$ & 10.75216 & -2.17341 & -1.67498 \\
\hline$r$ & 10.54916 & -3.36699 & -0.93978 \\
\hline C & 10.05924 & -4.36578 & 1.65041 \\
\hline $\mathrm{C}$ & 9.87136 & -3.98787 & 3.00248 \\
\hline $\mathrm{C}$ & 9.77747 & -1.80529 & 4.79048 \\
\hline C & 9.89023 & -0.39804 & 4.90405 \\
\hline $\mathrm{C}$ & 10.31870 & 1.97627 & 3.44415 \\
\hline C & 10.54421 & 2.47937 & 2.13931 \\
\hline $\mathrm{C}$ & 10.87158 & 1.73084 & -0.56069 \\
\hline C & 10.92369 & 0.63538 & -1.45697 \\
\hline $\mathrm{C}$ & 9.71334 & 0.07847 & -1.92722 \\
\hline C & 9.62687 & -1.40953 & -2.05741 \\
\hline C & 8.35086 & -2.00496 & -2.05521 \\
\hline $\mathrm{C}$ & 8.14964 & -3.19342 & -1.33142 \\
\hline $\mathrm{C}$ & 9.22813 & -3.75295 & -0.61924 \\
\hline $\mathrm{C}$ & 8.96712 & -4.27503 & 0.75839 \\
\hline $\mathrm{C}$ & 7.65890 & -4.17078 & 1.26975 \\
\hline $\mathrm{C}$ & 7.47455 & -3.77508 & 2.60670 \\
\hline $\mathrm{C}$ & 8.60210 & -3.51832 & 3.41039 \\
\hline $\mathrm{C}$ & 8.55194 & -2.36022 & 4.35570 \\
\hline $\mathrm{C}$ & 7.37962 & -1.57920 & 4.39670 \\
\hline $\mathrm{C}$ & 7.48816 & -0.18402 & 4.53806 \\
\hline $\mathrm{C}$ & 8.76768 & 0.40362 & 4.59899 \\
\hline $\mathrm{C}$ & 8.99702 & 1.67061 & 3.83610 \\
\hline $\mathrm{C}$ & 7.91829 & 2.20874 & 3.11053 \\
\hline $\mathrm{C}$ & 8.13782 & 2.67915 & 1.80323 \\
\hline $\mathrm{C}$ & 9.44056 & 2.63802 & 1.26999 \\
\hline $\mathrm{C}$ & 9.60998 & 2.22650 & -0.15793 \\
\hline $\mathrm{C}$ & 8.45883 & 1.88658 & -0.89663 \\
\hline $\mathrm{C}$ & 8.51425 & 0.80601 & -1.79557 \\
\hline $\mathrm{C}$ & 7.24273 & 0.20822 & -2.27382 \\
\hline $\mathrm{C}$ & 7.16538 & -1.19100 & -2.42009 \\
\hline $\mathrm{C}$ & 5.89981 & -1.80881 & -2.49980 \\
\hline $\mathrm{C}$ & 5.68593 & -3.07541 & -1.73521 \\
\hline $\mathrm{C}$ & 6.76100 & -3.57535 & -0.97149 \\
\hline $\mathrm{C}$ & 6.51562 & -4.05560 & 0.32974 \\
\hline $\mathrm{C}$ & 5.20192 & -4.02278 & 0.83859 \\
\hline $\mathrm{C}$ & 5.00808 & -3.57553 & 2.25163 \\
\hline $\mathrm{C}$ & 6.14828 & -3.24638 & 3.01419 \\
\hline $\mathrm{C}$ & 6.10070 & -2.14824 & 3.89693 \\
\hline $\mathrm{C}$ & 4.91516 & -1.38914 & 3.99556 \\
\hline $\mathrm{C}$ & 5.02680 & 0.09018 & 4.18583 \\
\hline $\mathrm{C}$ & 6.31485 & 0.66174 & 4.20250 \\
\hline $\mathrm{C}$ & 6.53349 & 1.87217 & 3.51605 \\
\hline $\mathrm{C}$ & 5.45631 & 2.48614 & 2.84956 \\
\hline $\mathrm{C}$ & 5.67570 & 2.90823 & 1.43253 \\
\hline $\mathrm{C}$ & 6.97230 & 2.78898 & 0.88787 \\
\hline $\mathrm{C}$ & 7.12790 & 2.37790 & -0.45106 \\
\hline $\mathrm{C}$ & 5.98457 & 2.08807 & -1.22565 \\
\hline $\mathrm{C}$ & 6.05372 & 0.95990 & -2.20391 \\
\hline $\mathrm{C}$ & 4.84845 & 0.37656 & -2.64283 \\
\hline $\mathrm{C}$ & 4.77143 & -1.02052 & -2.80350 \\
\hline $\mathrm{C}$ & 3.42833 & -1.65433 & -2.85120 \\
\hline $\mathrm{C}$ & 3.22783 & -2.85071 & -2.13497 \\
\hline $\mathrm{C}$ & 4.36717 & -3.40893 & -1.36232 \\
\hline
\end{tabular}




\begin{tabular}{|c|c|c|c|}
\hline $\mathrm{C}$ & 4.12234 & -3.88424 & -0.05867 \\
\hline $\mathrm{C}$ & 2.73954 & -3.79875 & 0.47356 \\
\hline $\mathrm{C}$ & 2.56008 & -3.35223 & 1.79642 \\
\hline $\mathrm{C}$ & 3.76017 & -3.02797 & 2.60900 \\
\hline $\mathrm{C}$ & 3.71584 & -1.91376 & 3.46855 \\
\hline $\mathrm{C}$ & 2.47666 & -1.09925 & 3.51023 \\
\hline $\mathrm{C}$ & 2.58415 & 0.28628 & 3.71011 \\
\hline $\mathrm{C}$ & 3.92378 & 0.90192 & 3.84404 \\
\hline $\mathrm{C}$ & 4.14182 & 2.15322 & 3.22842 \\
\hline $\mathrm{C}$ & 3.00752 & 2.75840 & 2.47554 \\
\hline $\mathrm{C}$ & 3.18633 & 2.98053 & 1.15187 \\
\hline $\mathrm{C}$ & 4.55887 & 2.91496 & 0.56958 \\
\hline $\mathrm{C}$ & 4.71473 & 2.50240 & -0.76948 \\
\hline $\mathrm{C}$ & 3.50851 & 2.17143 & -1.57382 \\
\hline $\mathrm{C}$ & 3.58658 & 1.14284 & -2.53075 \\
\hline $\mathrm{C}$ & 2.40063 & 0.60530 & -3.06908 \\
\hline $\mathrm{C}$ & 2.30986 & -0.87978 & -3.22686 \\
\hline $\mathrm{C}$ & 1.02757 & -1.47348 & -3.22381 \\
\hline $\mathrm{C}$ & 0.83351 & -2.67991 & -2.52112 \\
\hline $\mathrm{C}$ & 1.91346 & -3.24559 & -1.81105 \\
\hline $\mathrm{C}$ & 1.65320 & -3.74417 & -0.42381 \\
\hline $\mathrm{C}$ & 0.34523 & -3.61132 & 0.09147 \\
\hline $\mathrm{C}$ & 0.16814 & -3.13871 & 1.40710 \\
\hline $\mathrm{C}$ & 1.30145 & -2.85561 & 2.18948 \\
\hline $\mathrm{C}$ & 1.25692 & -1.65505 & 3.06837 \\
\hline $\mathrm{C}$ & 0.07345 & -0.88300 & 3.10989 \\
\hline $\mathrm{C}$ & 0.19630 & 0.5 & 666 \\
\hline $\mathrm{C}$ & 1.41418 & 1.1 & 1058 \\
\hline $\mathrm{C}$ & 1.63065 & 2. & 3.08129 \\
\hline $\mathrm{C}$ & 0.78777 & 3.1 & 0.71306 \\
\hline $\mathrm{C}$ & 2.04743 & 3.0 & 0.24109 \\
\hline $\mathrm{C}$ & 2.24336 & & -1.17372 \\
\hline $\mathrm{C}$ & 1.11091 & 2.3 & -1.96527 \\
\hline $\mathrm{C}$ & 1.20289 & 1. & -2.95093 \\
\hline $\mathrm{C}$ & -0.04454 & 0 & -3.49569 \\
\hline $\mathrm{C}$ & -0.15978 & -0 & -3.58076 \\
\hline $\mathrm{C}$ & -1.4 & -1.23673 & -3.66774 \\
\hline $\mathrm{C}$ & -1.67695 & -2.51215 & -2.93219 \\
\hline $\mathrm{C}$ & -0.54778 & -3.05587 & -2.14762 \\
\hline $\mathrm{C}$ & -0.79141 & -3.50663 & -0.84767 \\
\hline $\mathrm{C}$ & -2.16503 & -3.40612 & -0.30574 \\
\hline $\mathrm{C}$ & -2.31010 & -2.88544 & 1.08230 \\
\hline $\mathrm{C}$ & -1.14280 & -2.55416 & 1.80620 \\
\hline $\mathrm{C}$ & -1.15136 & -1.41367 & 2.64325 \\
\hline $\mathrm{C}$ & -2.35168 & -0.67685 & 2.78707 \\
\hline $\mathrm{C}$ & -2.29092 & 0.79721 & 3.00955 \\
\hline $\mathrm{C}$ & -0.95067 & 1.42928 & 2.97939 \\
\hline $\mathrm{C}$ & -0.81116 & 2.64806 & 2.42844 \\
\hline $\mathrm{C}$ & -1.94559 & 3.21537 & 1.66509 \\
\hline $\mathrm{C}$ & -1.67006 & 3.49973 & 0.23265 \\
\hline $\mathrm{C}$ & -0.36051 & 3.26407 & -0.24307 \\
\hline $\mathrm{C}$ & -0.15224 & 2.84951 & -1.57547 \\
\hline $\mathrm{C}$ & -1.32088 & 2.66405 & -2.46623 \\
\hline $\mathrm{C}$ & -1.26743 & 1.57377 & -3.46618 \\
\hline $\mathrm{C}$ & -2.46540 & 1.02287 & -3.93255 \\
\hline $\mathrm{C}$ & -2.55938 & -0.45456 & -4.03485 \\
\hline $\mathrm{C}$ & -3.91147 & -1.06430 & -4.02726 \\
\hline
\end{tabular}




\begin{tabular}{|c|c|c|c|}
\hline $\mathrm{C}$ & -4.11926 & -2.24164 & -3.29706 \\
\hline $\mathrm{C}$ & -2.99276 & -2.81304 & -2.52661 \\
\hline $\mathrm{C}$ & -3.24227 & -3.24091 & -1.20080 \\
\hline $\mathrm{C}$ & -4.62003 & -3.10202 & -0.64713 \\
\hline $\mathrm{C}$ & -4.77236 & -2.66212 & 0.68698 \\
\hline $\mathrm{C}$ & -3.54792 & -2.33520 & 1.46946 \\
\hline $\mathrm{C}$ & -3.56694 & -1.23916 & 2.35217 \\
\hline $\mathrm{C}$ & -4.82122 & -0.47192 & 2.51389 \\
\hline $\mathrm{C}$ & -4.75334 & 0.91819 & 2.67107 \\
\hline $\mathrm{C}$ & -3.42245 & 1.57669 & 2.69463 \\
\hline $\mathrm{C}$ & -3.24813 & 2.79812 & 1.99968 \\
\hline $\mathrm{C}$ & -4.38179 & 3.31322 & 1.19515 \\
\hline $\mathrm{C}$ & -4.15045 & 3.66957 & -0.14652 \\
\hline $\mathrm{C}$ & -2.75686 & 3.58797 & -0.66911 \\
\hline $\mathrm{C}$ & -2.56292 & 3.17852 & -2.07865 \\
\hline $\mathrm{C}$ & -3.75953 & 2.87112 & -2.88643 \\
\hline $\mathrm{C}$ & -3.71674 & 1.80060 & -3.79120 \\
\hline $\mathrm{C}$ & -4.98265 & 1.23234 & -4.30017 \\
\hline $\mathrm{C}$ & -5.08432 & -0.24766 & -4.42673 \\
\hline $\mathrm{C}$ & -6.35989 & -0.83964 & -4.43337 \\
\hline $\mathrm{C}$ & -6.57653 & -2.08902 & -3.65374 \\
\hline $\mathrm{C}$ & -5.49786 & -2.63404 & -2.93216 \\
\hline $\mathrm{C}$ & -5.72509 & -3.06386 & -1.52406 \\
\hline $\mathrm{C}$ & -7.02483 & -2.98142 & -0.99675 \\
\hline $\mathrm{C}$ & -7.18837 & -2.54910 & 0.40960 \\
\hline $\mathrm{C}$ & -6.03596 & -2.22798 & 1.14606 \\
\hline $\mathrm{C}$ & -6.10026 & -1.10473 & 2.12207 \\
\hline $\mathrm{C}$ & -7.29525 & -0.36972 & 2.24134 \\
\hline $\mathrm{C}$ & -7.21968 & 1.11093 & 2.37559 \\
\hline $\mathrm{C}$ & -5.95727 & 1.73124 & 2.39395 \\
\hline $\mathrm{C}$ & -5.75989 & 2.98499 & 1.62124 \\
\hline $\mathrm{C}$ & -6.82754 & 3.48216 & 0.86575 \\
\hline $\mathrm{C}$ & -6.55703 & 3.91404 & -0.52826 \\
\hline $\mathrm{C}$ & -5.24415 & 3.80 & -1.03641 \\
\hline $\mathrm{C}$ & -5.06589 & 3.4 & -2.47017 \\
\hline $\mathrm{C}$ & -6.2( & 3.1 & -3.24910 \\
\hline $\mathrm{C}$ & -6.1 & 1.9 & 9956 \\
\hline $\mathrm{C}$ & -7.4 & 1.3 & -4.62721 \\
\hline $\mathrm{C}$ & -7.54 & 0.00 & -4.74117 \\
\hline $\mathrm{C}$ & -8.88561 & -0.62091 & -4.78425 \\
\hline $\mathrm{C}$ & -9.10233 & -1.86152 & -3.99336 \\
\hline $\mathrm{C}$ & -7.95967 & -2.39478 & -3.21845 \\
\hline $\mathrm{C}$ & -8.17867 & -2.83944 & -1.91150 \\
\hline $\mathrm{C}$ & -9.54863 & -2.79902 & -1.35382 \\
\hline $\mathrm{C}$ & -9.71788 & -2.38750 & 0.06545 \\
\hline $\mathrm{C}$ & -8.50665 & -2.02849 & 0.83867 \\
\hline $\mathrm{C}$ & -8.56046 & -0.95748 & 1.73646 \\
\hline $\mathrm{C}$ & -9.82556 & -0.19843 & 1.87318 \\
\hline $\mathrm{C}$ & -9.74174 & 1.28243 & 1.97951 \\
\hline $\mathrm{C}$ & -8.39995 & 1.90449 & 1.95911 \\
\hline $\mathrm{C}$ & -8.20503 & 3.07010 & 1.21076 \\
\hline $\mathrm{C}$ & -9.33968 & 3.63788 & 0.45374 \\
\hline $\mathrm{C}$ & -9.08054 & 4.12771 & -0.92641 \\
\hline $\mathrm{C}$ & -7.70473 & 3.99890 & -1.46480 \\
\hline $\mathrm{C}$ & -7.53027 & 3.61719 & -2.79843 \\
\hline $\mathrm{C}$ & -8.72328 & 3.35315 & -3.63559 \\
\hline $\mathrm{C}$ & -8.67865 & 2.20226 & -4.57617 \\
\hline
\end{tabular}




\begin{tabular}{|c|c|c|c|}
\hline $\mathrm{C}$ & -9.89298 & 1.64923 & -5.02534 \\
\hline$c$ & -10.00457 & 0.16237 & -5.12854 \\
\hline $\mathrm{C}$ & -10.41678 & -2.19497 & -3.61272 \\
\hline $\mathrm{C}$ & -10.65058 & -2.69452 & -2.22355 \\
\hline $\mathrm{C}$ & -10.97882 & -1.92642 & 0.48925 \\
\hline $\mathrm{C}$ & -11.03595 & -0.77401 & 1.43880 \\
\hline $\mathrm{C}$ & -10.86749 & 2.04846 & 1.61883 \\
\hline $\mathrm{C}$ & -10.65545 & 3.28830 & 0.81083 \\
\hline $\mathrm{C}$ & -10.16905 & 4.25228 & -1.81163 \\
\hline $\mathrm{C}$ & -9.97938 & 3.84977 & -3.23788 \\
\hline $\mathrm{C}$ & 11.42224 & 1.38983 & 4.24437 \\
\hline $\mathrm{H}$ & 12.41191 & 1.82589 & 4.22581 \\
\hline $\mathrm{C}$ & 11.89804 & 2.46338 & 1.53360 \\
\hline $\mathrm{H}$ & 12.77375 & 2.67779 & 2.13126 \\
\hline $\mathrm{C}$ & 12.05293 & & 0.25308 \\
\hline $\mathrm{H}$ & 13.05161 & & -0.15769 \\
\hline $\mathrm{C}$ & 12.1 & -0.1 & -1.60961 \\
\hline $\mathrm{H}$ & 13.1 & 0.2 & -1.58034 \\
\hline $\mathrm{C}$ & 12.07700 & -1.50634 & \\
\hline $\mathrm{H}$ & 12. & -2.0 & 483 \\
\hline $\mathrm{C}$ & 11.2 & & \\
\hline $\mathrm{H}$ & 12.0 & & \\
\hline $\mathrm{C}$ & 10. & & \\
\hline $\mathrm{H}$ & 11. & & 22 \\
\hline $\mathrm{C}$ & 11. & & 85 \\
\hline $\mathrm{H}$ & 11. & & 76 \\
\hline $\mathrm{C}$ & & & 39 \\
\hline $\mathrm{H}$ & 12.2 & & .63826 \\
\hline $\mathrm{C}$ & 11.65161 & 82 & -0.16516 \\
\hline 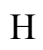 & 12. & -4.0 & -0.55572 \\
\hline 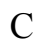 & -11.53100 & 4.3 & -1.22654 \\
\hline 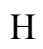 & -12. & 4.6 & -1.81416 \\
\hline C & -11. & 3.8 & 0.00832 \\
\hline $\mathrm{H}$ & -12.7 & 3.8 & 0.39045 \\
\hline $\mathrm{C}$ & -12.18883 & $1.3^{\circ}$ & 1.63148 \\
\hline $\mathrm{H}$ & -13.09983 & 1.95966 & 1.64614 \\
\hline 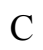 & -12.2 & 0.04 & 1.55110 \\
\hline 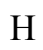 & -13.2 & -0.4 & 1.50430 \\
\hline 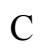 & -11.14969 & 3.5 & -4.09194 \\
\hline $\mathrm{H}$ & -12.06633 & 4.09725 & -4.00054 \\
\hline $\mathrm{C}$ & -11.10959 & 2.49184 & -4.93323 \\
\hline $\mathrm{H}$ & -11.99457 & 2.24103 & -5.50198 \\
\hline $\mathrm{C}$ & -11.33519 & -0.49263 & -5.11140 \\
\hline $\mathrm{H}$ & -12.17468 & -0.04351 & -5.62429 \\
\hline $\mathrm{C}$ & -11.53008 & -1.60506 & -4.39473 \\
\hline $\mathrm{H}$ & -12.52274 & -2.03116 & -4.34342 \\
\hline $\mathrm{C}$ & -12.00328 & -2.63790 & -1.61892 \\
\hline $\mathrm{H}$ & -12.88085 & -2.83154 & -2.22055 \\
\hline $\mathrm{C}$ & -12.15789 & -2.27908 & -0.33954 \\
\hline $\mathrm{H}$ & -13.15755 & -2.19217 & 0.06348 \\
\hline . & 0.44587 & 4.62583 & 2.40701 \\
\hline $\mathrm{N}$ & 0.51545 & 3.27165 & 2.16903 \\
\hline 1. & 1.56970 & 3.52066 & 4.41331 \\
\hline 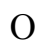 & 2.09103 & 2.96071 & 5.33368 \\
\hline
\end{tabular}

